\title{
Abstracts for the 35th Human Genetics Society of Australasia Annual Scientific Meeting, Gold Coast, Australia
} July 31-August 3, 2011

\section{Invited Abstracts}

\section{CONTROL OF APOPTOSIS BY THE BCL-2 PROTEIN FAMILY AND ITS IMPLICATIONS FOR CANCER DEVELOPMENT AND THERAPY}

\section{J.M. Adams}

Walter and Eliza Hall Institute of Medical Research, Australia

It is now accepted that a central step in the development of most if not all cancers is impairment of apoptosis, the natural process of cell death. This essential biological process is generally controlled by the relative abundance and interactions of three factions of the Bcl-2 protein family. Apoptosis is signally by distant $\mathrm{Bcl}-2$ relatives, the ' $\mathrm{BH} 3$-only proteins', but requires their activation of the more closely related Bax or Bak, which then oligomerize and elicit cell death by disrupting the mitochondrial outer membrane, thereby unleashing a proteolytic cascade. Bcl-2 and its closest relatives (e.g., Bcl-xL and Mcl-1) prevent apoptosis by preventing the activation and oligomerization of Bax and Bak. The steps by which the apoptotic switch is flipped are now being revealed. Apoptosis is constrained in many cancer cells; for example, by elevated expression of Bcl-2, reducing the effectiveness of conventional cancer therapies. Hence, there is now intense interest in the prospect of developing, as novel anti-cancer agents, small synthetic molecules that antagonize one of more pro-survival family members as do their natural ligands, the BH3-only proteins. The first of these 'BH3 mimetics', such as Abbott's ABT-737, which targets three of the pro-survival proteins, have shown considerable promise in pre-clinical studies and also encouraging results in the first clinical trials, particularly in cancers where $\mathrm{Bcl}-2$ is overexpressed.

\section{GENETICS AND CARDIOMYOPATHIES: A COLLABORATIVE} MODEL OF CARE

\section{J. Atherton}

Royal Brisbane and Women's Hospital, Australia

The classification of cardiomyopathies is based upon phenotype and includes hypertrophic, dilated and arrhythmogenic right ventricular cardiomyopathies. Genetic studies have identified multiple disease genes for all the inherited cardiomyopathies and have provided mechanistic insights into disease pathogenesis. However, apart from a few notable exceptions, prognosis and management decisions are largely guided by phenotype lending support to the current morphological classification. Given that most of the familial cardiomyopathies have an autosomal dominant pattern of inheritance and that effective screening and treatment strategies are available, it is recommended that all 1st-degree relatives should undergo clinical screening, which usually involves an ECG, echocardiogram and review by a cardiologist. However, phenotypic variation both between and within families with a variable age of disease-onset mandates the need for periodic, long-term, clinical screening. Diagnostic genetic testing is now commercially available for a number of the cardiomyopathies. If a disease-causing mutation is identified in the proband, then predictive testing in relatives allows long-term clinical screening to be focussed on mutation carriers. However, despite the established clinical utility of genetic testing, its diffusion into clinical practice has been variable for a number of reasons including lack of awareness by referring clinicians, variable access to specialized clinical genetic services and reimbursement processes. Ideally, a collaborative model of care involving cardiology and clinical genetics will allow more consistent access to genetic testing using accepted criteria based upon pickup rates, clinical utility and cost.

FETAL DETERMINANTS OF HUMAN FERTILITY AND TESTICULAR CANCER

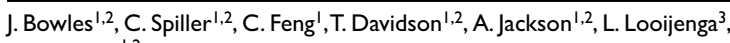
P. Koopman ${ }^{1,2}$

${ }^{1}$ Division of Molecular Genetics and Development, Institute for Molecular Bioscience, University of Queensland, Australia

${ }^{2}$ Australian Research Council Centre for Excellence in Biotechnology and Development, Australia

${ }^{3}$ Department of Pathology, Josephine Nefkens Institute, Erasmus MC, University of Medical Center Rotterdam, Netherlands

In the mouse fetal gonad, germ cells commit to a female or male sexual fate on the basis of environmental cues, rather than XX or XY chromosome constitution. Germ cells in a developing ovary enter into meiosis, hence committing to the female fate or oogenesis. In a developing testis, germ cells do not enter meiosis during fetal life but they do stop proliferating and arrest in G0/G1 (mitotic quiescence), hence committing to the male fate or spermatogenesis. In recent years we have shown that germ cells in a female mouse embryonic gonad are triggered to enter meiosis by the potent signaling molecule retinoic acid (RA). RA induces germ cells to express a key gene, Stra8, which encodes a protein essential for initiation of meiosis. In the developing testis, germ cells avoid entering meiosis because RA is actively degraded by a cytochrome P450 enzyme, CYP26B1. Hence, CYP26B1 is a 'meiosisinhibiting' substance by virtue of its ability to degrade the meiosis inducer, RA. We have also found that fibroblast growth factor 9 (FGF9), a signaling molecule produced by pre-Sertoli cells, acts in the male gonad to inhibit meiosis and to push germ cells towards the male fate. 
We are now studying pathways downstream of these two triggering factors. Our studies are relevant to an understanding of fertility, infertility and the genesis of testicular germ cell tumors, the most common cancer of young men.

\section{LONG QT SYNDROME - A NEW ERA IN GENETIC TESTING D. Du Sart \\ Molecular Genetics, Victorian Clinical Genetics Services Pathology, MCRI, Australia}

Inherited cardiac arrhythmias, particularly Long QT syndrome (LQT), which involve abnormal electrical activity in a structurally normal heart, are conditions that cause unexpected sudden death occurring during physical activity, at times of emotional stress or even while asleep. The estimated prevalence is 1 in 3000; usually autosomal dominant inheritance and the symptoms include fainting, epilepsy and sudden death, particularly in young people. Genetic testing for these disorders can identify individuals and their relatives who have the condition with greater precision than routine cardiac testing and has therefore become an essential component of clinical management. Affected individuals need lifelong treatment and unaffected individuals can be freed from unnecessary follow-up testing or inappropriate medication, typically beta-blockers, and sometimes implanted devices to reduce risk of sudden death. Genetic testing for LQT raises many complex issues in both the testing process and clinical management of families. There are currently 12 genes associated with LQT that are involved in forming the membrane ion channels in cells. Genetic testing routinely involves screening six of these, namely KCNQ1, $\mathrm{KCNH} 2$, SCN5A, KCNE1, KCNE2 and KCNJ2, accounting for the majority of cases. The six genes cover an enormous 30,000 bases of DNA for screening. Finding cost effective, efficient methods for testing these genes pose a technological challenge for many laboratories. In addition $\sim 20 \%$ of cases are found to be compound heterozygous for two or more mutations in the same or different genes, as well as many common variants which could impact on the QT length. Recently, mutations in a secondary transcript of $\mathrm{KCNH} 2$ were identified in patients who did not have mutations in the common transcript usually tested. Again, these multigenic, multifactorial findings pose enormous challenges in defining the pathogenic gene changes for testing and managing of family members. This presentation will discuss ways for dealing with the complexity of genetic testing in LQT.

\section{DOES THE AXIN GENE HAVE A ROLE IN POLYPOSIS?}

D. Du Sart', J. Marum', R. Scott ${ }^{2}$, F. Macrae ${ }^{3}$

${ }^{1}$ Molecular Genetics, Victorian Clinical Genetics Services Pathology, MCRI, Australia

${ }^{2}$ Hunter Area Pathology Service, John Hunter Hospital, Newcastle, Australia

${ }^{3}$ Colorectal Medicine and Genetics, Royal Melbourne Hospital, Melbourne, Australia

The major genetic basis of familial polyposis is germline mutation $\mathrm{s}$ of the APC gene. As part of the Wnt-pathway, APC protein forms a complex with the cytoplasmic proteins $\beta$-catenin, axin and glycogen synthase kinase- $3 \mathrm{~b}$ and this complex induces $\beta$-catenin degradation. Without $\beta$-catenin degradation, the $\beta$-catenin-Tcf complex leads to transcriptional activation of several genes and oncogenes like c-myc, a transcription factor for several genes controling cell growth and division. This cascade of events ultimately leads morphologically to adenomatous polyp formation. A second mechanism leading to familial polyposis involves the base-excision repair gene, MYH. Patients who carry two germline mutations in the MYH gene have an increased rate of somatic APC gene mutations, which predisposes to polyp formation and cancer progression as a consequence of non-functional APC protein. The Axin protein is a scaffolding protein housing several critical binding domains for proteins within the Wnt-pathway. Axin recruits unphosphorylated $\beta$-catenin to the destruction complex. It binds to GSK-3 $b$ to enable phosphorylation of $\beta$-catenin. It binds to 20 -amino acid repeats of APC to enable phosphorylation of APC, which increases APC affinity for $\beta$-catenin; phosphorylated APC removes phosphorylated $\beta$-catenin for degradation. As a critical component of the Wnt-pathway, would germline mutations in this gene lead to familial polyposis? We have screened a cohort of patients with polyposis and no germline APC or MYH mutations for mutations in Axin. No deletion or duplication mutations were identified in 200 patients. No truncating mutations, but however, four novel germline missense mutations and one possible splicesite mutation were identified in 60 patients sequenced so far. The interplay between Axin1 and Axin2 within the Wnt-pathway and tumor pathogenesis will be discussed. These data suggests that mutations in Axin may indeed have a role in polyposis.

\section{CHANGING DEMANDS IN THE DISAGONOSIS OF POMPE DISEASE DUE TO THE INTRODUCTION OF ENZYME REPLACEMENT THERAPY}

M. Fietz, J. Pacyna, T. Pyragius, S. Williams, P. Clements, J. Fletcher

National Referral Laboratory, Women's and Children's Hospital, Australia

Pompe disease (glycogen storage disease type II, acid maltase deficiency) is an inherited disorder caused by a deficiency of the lysosomal enzyme $\alpha$-glucosidase. The phenotype ranges from infantile onset of hypotonia and cardiomyopathy to the adult-onset form, which presents with diaphragmatic and proximal muscle weakness, particularly affecting the psoas muscles. The availability of Department of Health and Ageing-funded enzyme replacement therapy (ERT) for patients affected by infantile Pompe disease, has led to markedly altered demands on the diagnostic laboratory. Critical to the success of such therapy, which is shown to greatly alleviate the clinical presentation and significantly increase the lifespan of treated patients, is the early commencement of treatment. To enable treatment from the youngest possible age, it is essential to provide a rapid and definitive diagnosis of Pompe disease. Diagnosis is complicated by the expression of an isozyme in neutrophils and by the observation of false-positive results using analysis of dried blood spots, the current first-stage diagnostic test. Patients who produce little or no endogenous $\alpha$-glucosidase protein have been shown to develop strong immunological responses to therapy, necessitating the introduction of CRIM (cross-reacting immunological material) testing of patient samples, in order to determine which infants should be offered immunosuppression at ERT onset. As there is a relatively strong genotype/phenotype correlation with Pompe disease, there is clinical demand for mutation studies of the GAA gene. Finally, upon the commencement of therapy, it is extremely helpful to have biochemical markers in order to monitor the progress of the patient. This presentation will discuss the developments that have been introduced by our laboratory in order to meet these demands and how these have assisted in patient management.

\section{SEARCHING FOR GOLD: STRATEGIES, SUCCESSES} AND SURPRISES

A. Hackett

Newcastle University, Australia

The Genetics of Learning Disability (GOLD) service is a NSW statewide service, based at Hunter Genetics in Newcastle. It was established in the year 2000 as an expansion of the Fragile X Program, with an aim to provide testing and family genetic counseling for both Fragile X syndrome and X-linked Intellectual Disability (XLID). Since the discovery of the FMR1 gene (Fragile $\mathrm{X}$ syndrome), clinicians and scientists have been searching for the other causes of XLID. Currently 92 XLID genes have been identified, yet many more remain to be discovered. The GOLD service has been involved with over 300 XLID families, some for more than 20 years. The majority of families simply want to know what the cause of the ID is and the chance of reoccurrence. Our aim is to help them, by continuing to search for the cause of the family's ID, as long as the family wish. For some families, the answers have come in weeks, yet for others it has taken more than 20 years. And despite our best efforts, there are still many families without an answer. This presentation looks at our and the family's 'search for GOLD'. While our successes have brought joy to the families, staff and scientists, false leads and ambiguous results have caused confusion and frustration, and incorrect results have had devastating consequences. We present some of the more memorable surprises, the successes and lessons learnt along the way. 
DIETARY COMPLIANCE AND OUTCOME IN CLASSICAL GALACTOSEMIA

U. Hendroff
National Centre for Inherited Metabolic Disorders, Children's University Hospital, Dublin,
Ireland

Classical galactosemia $(\mathrm{Gal})$, an autosomal recessive inherited disorder of galactose metabolism is characterized by a deficiency in the enzyme: galactose-1-phosphate uridyl transferase (GALT). In Ireland the incidence is reported as $1 / 19,000$ births with increased frequency of $1 / 450$ births in the Irish Traveller population. Strict dietary exclusion of galactose is life saving in neonates and is the mainstay of treatment throughout the lifecycle. Despite early treatment and good dietary compliance multiple studies have reported long-term complications, including cognitive dysfunction, neurological impairment, speech difficulties, growth retardation and ovarian failure. Recent studies and reports have suggested that patients following a less restrictive galactosefree diet may benefit from a more favorable outcome. A recent study at this centre involved a detailed retrospective chart review of $30 \mathrm{Gal} \mathrm{sub-}$ jects (11 females, 19 male) aged between 6 months to 26 yrs from 14 families. ${ }^{1} 24 / 30(80 \%)$ were homozygous for the Q188R mutation and all 30 subjects had $<0.5 \%$ GALT activity. Outcome was compared between the sibling pairs for IQ, speech and language delay, neurological examination and brain MRIs with reported dietary compliance, mean RBC Gal-1-P and urine galactitol levels. 163 diet histories were analyzed (range 1-9 per patient) and mean galactose intake estimated. Subjects with intakes $<20 \mathrm{mg}$ galactose/day were grouped as compliant while those $>20 \mathrm{mg} /$ day noncompliant. There were no significant differences in outcome between first and subsequent siblings and no correlation seen between mean RBC Gal-1-P or urine galactitol levels. Also no significant differences were seen between mean galactose intake and outcomes. The subjects noncompliant with diet and on higher galactose intakes did not exhibit an increased incidence of complications. Subjects very compliant with diet had the poorest outcomes. Current studies underway further highlight the possible requirement for some dietary galactose to avoid galactose deficiency in classical galactosemia.

${ }^{1}$ Journal of Pediatrics 2009; 154:721-726.

\section{CONGENITAL HEART DISEASE — ARE WE THERE YET?}

E. Kirk

Department of Medical Genetics, Sydney Children's Hospital, Australia

When we think of cardiac genetics, the focus tends to be on inherited cardiomyopathies and disorders of electrical conduction. But congenital heart disease (CHD) is more common than those conditions combined, and is largely genetic in origin. So why are cardiac genetics clinics not (yet) overflowing with patients with CHD? Will we get to that point, and if so, when? Part of the answer, of course, is that unlike disorders like hypertrophic cardiomyopathy or long QT syndrome, the bulk of $\mathrm{CHD}$ is non-Mendelian, and genetic testing currently has no role. Nonetheless, there is considerable demand from the parents of children with CHD for answers to the questions 'Why did this happen?' and 'Will it happen again?' An accurate answer requires evaluation for subtle syndromal forms of CHD, consideration of the possibility of a Mendelian form for which genetic testing may be helpful, and a sound understanding of the genetics of complex disease and the application of empiric risk data. In this talk, I will review recent progress in both Mendelian and non-Mendelian CHD genetics, including recent animal studies that point to great complexity in the multifactorial causation of CHD. Indications for genetic testing in familial CHD will be discussed.

\section{THE GENETICS OF COMPLEX TRAITS:}

\section{THE GWAS REVOLUTION AND BEYOND}

\section{N. Martin \\ Queensland Institute of Medical Research, Brisbane, Australia}

Most of the disease burden in developed countries is from disorders of multifactorial etiology. That they tend to 'run in families' can be due to shared genes or shared environments and twin studies have played a major role in establishing the relative roles of these broad sources of variation. The challenge now is to find the particular genes and pathways underlying disease risk, and how they interact with environments we can measure, and the residual which we cannot. After decades of little progress, there has been an explosion of discoveries in complex trait genetics since the advent of SNP chips in 2005. Illustrations across a wide range of traits and conditions will be given.

\section{PATIENT EMPOWERMENT: TOWARD OUTCOME MEASUREMENT IN GENETIC COUNSELING PRACTICE}

M. McAllister

Universities of Cardiff \& Manchester, Cardiff \& Manchester, United Kingdom

Introduction: Patient outcomes from using clinical genetics services are multidimensional and difficult to measure. A program of research to address the problem of outcome measurement conducted in Manchester, UK 2003-2010 will be summarized. Methods and Results: A systematic review, a Delphi survey and preliminary qualitative research identified six outcome domains as valued: Knowledge of the condition, quality of life, ability to cope, perceived personal control, decision-making, and accuracy of diagnosis. The qualitative element to this program will be described in detail. Interviews and focus groups were conducted with 31 patients, 34 patient representatives, 24 health professionals and 4 service commissioners, and analyzed using grounded theory. A model of empowerment was developed summarizing the patient benefits from using clinical genetics services, with five dimensions: cognitive control, decisional control, behavioral control, emotional regulation and hope. Process attributes and interventions that may contribute to patient empowerment in clinical genetics were also identified. When existing outcome measures identified in the systematic review were compared with the empowerment construct, it became clear that existing measures could not capture all the important patient benefits. Findings were then used to develop a new Patient Reported Outcome Measure to capture empowerment - the Genetic Counseling Outcome Scale (GCOS-24), which was shown to have good reliability, internal consistency, construct validity and sensitivity to change (Cohen's $d=0.70)$. Conclusion: The Genetic Counseling Outcome Scale has potential for use as a patient reported outcome measure to evaluate genetic counseling interventions in both research and clinical contexts.

\section{COURAGE IN DISCOVERY}

\section{J. Pearn}

Royal Children's Hospital, Brisbane, Australia

Human genetics spans every facet of biology from molecular structure, through laboratory and clinical practice, to psychology and anthropology. The history of human genetics has been punctuated by paradigm shifts in knowledge. Each such new concept encountered with supplies, often with perplexity or frank incredulity and sometimes with disbelief. The datum milestones along the path leading to our present corpus of genetic knowledge are well known to all geneticists; but almost all such new discoveries were threats to the received wisdom of their time and place. Researchers, scientists and clinicians encountering such new and often heretical paradigm shifts required courage to accept the possibility if not the fact as such themes post-Aristotelian genetic theories, Darwinian evolution, Mendel's first and second laws, linkage, mitochondrial genes, uni-parental disomy and infamy. Many of these great steps in the understanding of fundamental biology were encountered initially by unsolicited, often incidental, individual observation. Such required considerable courage to recognise instances where perceived wisdom appeared to be violated. Features of the path to contemporary genetic knowledge are illustrations of the courage needed to contemplate, initially in speculative thought, experiments of new possibilities. Herein lies a message for us all. 


\section{PROTECTING THE HUMAN GENOME}

D.J. Richard

Queensland University of Technology, Australia

The damage response pathway is a crucial component of the surveillance network that maintains the stability and integrity of our genome. Maintenance of genomic stability is critical for cellular homeostasis, with genomic instability leading to cancer or cell death. Cells respond to DNA damage by activating a complex DNA-damage-response pathway that includes cell cycle arrest, the transcriptional and posttranscriptional activation of a subset of genes including those associated with DNA repair, and under some circumstances, the triggering of programmed cell death. This means that the damage signal is conveyed rapidly and precisely to numerous pathways across the affected cell. The cellular resources dedicated to this response underlies the importance of maintaining the genetic code. Genetic defects that disturb the damage response pathway cause severe syndromes characterized by neurodegeneration, immunodeficiency, chromosomal instability and a striking predisposition to cancer. Our work centres on the discovery of new components of these DNA repair pathways and trying to piece together how these proteins and systems function together to maintain genomic stability. Once such protein that we recently identified was hSSB1. hSSB1 was originally discovered as a homologue to the Sulfolobus solfataricus SSB, which is a DNA repair protein. We have demonstrated that hSSB1 functions directly in the repair of double strand DNA breaks by initially binding the break site and then recruiting the essential MRN complex. Once recruited hSSB1 further stimulates the MRN nuclease activity, a process required to initiate homologous recombination. This then allows the initiation of the signaling and repair processes. Cells lacking hSSB1 fail to initiate any response to ionising radiation and will fail to repair the damage. Interestingly further work from our team now reveals that hSSB1 is also required to repair oxidative DNA damage. This data demonstrates the critical role hSSB1 plays in maintaining genome stability.

\section{A CLINICAL ROLE FOR COMMON GENOMIC VARIANTS IN FAMILIAL BREAST CANCER}

S. Sawyer', J. McKinley', G. Mitchell', G. Chenevix-Trench², M. Harris², G. Lindeman ${ }^{4}$, P.A. James ${ }^{1,5}$

${ }^{1}$ Peter MacCallum Cancer Institute, Australia

${ }^{2}$ Queensland Institute of Medical Research, Australia

${ }^{3}$ Monash Medical Clinic, Australia

${ }^{4}$ Melbourne Health, Australia

${ }^{5}$ Victorian Clinical Genetics Services, Australia

More than 100 Genome-wide association studies have been published focusing on types of cancer. The results have provided new insights into the biology of cancer and opened avenues for novel research but it is less clear whether there are direct clinical applications for common predisposition variants. Many cancer GWAS are large with high levels of confidence for the reported associations, but overall effect sizes are small (median OR1.27), reported associations are frequently unable to be replicated and in most cases the total attributable risk remains low. In the field of Familial Cancer however the possibility remains that common variants may provide clinically important information in the large majority of families where the history is not due to a high penetrance mutation. We examined the role of risk-associated common variants in the etiology of familial breast cancer in the Victorian FCC Translational Hereditary Breast and Ovarian Cancer cohort. Extended profiling for 24 well-validated breast cancer predisposition SNPs in a group of 1361 index cases from high risk breast cancer families, previously screened for BRCA1/2 mutations, and 1068 controls. The cases demonstrate a highly significant excess of risk alleles and increased average polygenic risk $(p<1 \times 10-59)$. More than a third of the index cases had polygenic risk scores in the highest $10 \%$ of the control population. A high risk SNP profile defined a clinically distinct group of patients that had a reduced chance of a BRCA1/2 mutation and a low residual risk of ovarian cancer, but a significantly increased rate of second primary breast cancer. This data suggests that incorporating common variants into current practice has the potential to improve both our understanding of breast cancer in families and the effectiveness of recommended risk-management strategies.

\section{SUDDEN CARDIAC DEATH IN THE YOUNG}

C. Semsarian

University of Sydney \& Centenary Institute, Australia

Sudden cardiac death (SCD) is a tragic and devastating complication of a number of cardiovascular diseases. Although coronary artery disease accounts for a majority of these deaths across all ages, many other etiologies contribute to this problem when it occurs in the young (age $\leq 35$ years), where coronary artery disease is far less common. ${ }^{1}$ Specifically, genetic heart disorders are an important cause of SCD in the young. While pharmacological therapies have made some impact on prevention of SCD, the introduction of implantable cardioverter-defibrillator (ICD) therapy has been the single major advance in the prevention of SCD in the young. ${ }^{2}$ In addition, the awareness that most causes of SCD in the young are inherited means family screening of relatives of young SCD victims allows identification of previously unrecognized at-risk individuals, thereby enabling prevention of SCD in relatives. The role of genetic testing, both in living affected individuals and in the setting of a 'molecular autopsy', is emerging as a key factor in early diagnosis of an underlying cardiovascular genetic disorder. ${ }^{3}$ In 2010, a 3-year, prospective, populations-based, Trans-Tasman study (ANZSCD Study) has begun focusing on the clinical, pathological and genetic basis of SCD in the young, and how clinical and genetic screening of relatives can lead to improved clinical outcomes including prevention of SCD. Understanding the genetic basis of SCD, investigating the molecular mechanisms that lead from the gene defect to the clinical phenotype, and elucidating the specific environmental triggers for SCD, will most likely lead to further key improvements in the prevention of SCD in the young.

${ }^{1}$ Medical Journal of Australia 2004; 180:110-112.

${ }^{2}$ Theraputic Advances in Cardiovascular Disease 2009; 3:145-155.

${ }^{3}$ Internal Medicine Journal 2007; 37:32-37.

\section{PRADER-WILLIS SYNDROME [PWS] — IS BEHAVIORAL} MODIFICATION POSSIBLE?

E. Smith ${ }^{1,3}$, S. Einfeld ${ }^{1,2}$, A. Guastella ${ }^{2}$, I. McGregor ${ }^{1}$, K. Steinbeck ${ }^{1,3}$, S. Horstead ${ }^{2}$ S. Dodd ${ }^{2}$

${ }^{1}$ University of Sydney, Australia

${ }^{2}$ Brain and Mind Research Institute, Australia

${ }^{3}$ Children's Hospital at Westmead, Australia

Rationale: There is a distinct behavioral phenotype in PWS, encompassing temper tantrums, perseveration, obsessive compulsive, manipulative conduct, sudden aggression and abnormal food seeking with pica. It is the behavioral aspects which are of most concern for carers. Postmortem studies on brain tissue have shown a deficiency of oxytocin producing cells in the hypothalamus in PWS. A priori administration of oxytocin could alleviate behavioral problems, which would be worthwhile in terms of management. Methods: A clinical trial was set up with DNA proven subjects with PWS, administering oxytocin as a nasal spray (24IU for participants aged 16 years and over; 18IU for those under 16 years). The study comprises enrolment of 30 PWS subjects, aged 12-30 years, in a randomized controled crossover within-subjects double blind trial (each subject is its own control) with OT (or placebo) administered for 8 weeks (1st arm), followed by a wash out period of 2 weeks, and then a further 8 weeks of OT nasal spray (or placebo) (2nd arm), with assessments made at standard time points. The modules used for testing include well validated items from the Developmental Behavior Checklist, Yale Brown Obsessive Compulsive Scale, the Dykens Hyperphagia Questionnaire, Epworth Sleepiness Scale and Reading the Mind in the Eye test (RMET). Results: To date, 8 clients have completed both arms of the trial, 3 have completed 1 st arm, and 5 are ready to start. Codes have not yet been broken, but anecdotally, some have noticed a change in behavior while others have not. Conclusion: It is too early to say whether or not the 
trial is successful. New evidence suggests that administration of OT alters innate OT production, so that increasing the dose of OT may be more effective in inducing alteration of behavior.

\section{GASTRIC ADENOCARCINOMA AND PROXIMAL POLYPOSIS OF THE STOMACH (GAPPS): A NEW AUTOSOMAL DOMINANT SYNDROME}

D.Worthley ${ }^{2}$, K. D. Phillips', N.Wayte ${ }^{3}$, K.A. Schrader ${ }^{4}$, S. Healey ${ }^{3}$, P. Kaurah', A. Shulkes ${ }^{5}$, F. Grimpen ${ }^{6}$,A. Clouston ${ }^{6}$, D. Moore ${ }^{7}$, D. Cullen 8 , D. Ormonde ${ }^{8}$, D. Mounkley ${ }^{9}$, X.Wen ${ }^{10}$, N. Lindor ${ }^{\prime \prime}$, F. Carneiro ${ }^{10}$, D. G. Huntsman ${ }^{4}$, G. Chenevix-Trench ${ }^{3}$, G. K. Suthers ${ }^{1,12}$

${ }^{1}$ SA Clinical Genetics Service, SA Pathology, Adelaide, Australia

2 Division of Digestive and Liver Diseases, Columbia University, United States

${ }^{3}$ Queensland Institute of Medical Research, Australia

${ }^{4}$ Hereditary Cancer Program, BC Cancer Agency, BC, Canada

${ }^{5}$ Department of Surgery, University of Melbourne, Australia

${ }^{6}$ Royal Brisbane and Women's Hospital, Australia

7 Women's and Children's Hospital, Australia

${ }^{8}$ St John of God Hospital, Australia

${ }^{9}$ Flinders Medical Centre, Australia

${ }^{10}$ Institute of Molecular Pathology and Immunology, University of Porto, Portugal

${ }^{11}$ Department of Medical Genetics, Mayo Clinic College of Medicine, United States

${ }^{12}$ Department of Paediatrics, University of Adelaide, Australia

Fundic gland polyps (FGPs) are the most common gastric polyp. Sporadic FGPs are usually small, sparse, and restricted to the gastric body and fundus. FGPs are directly associated with chronic proton pump inhibitor therapy and inversely associated with $\mathrm{H}$. pylori infection, suggesting a possible connection between FGPs and disturbed gastric acid homeostasis. Fundic gland polyposis is also a feature of some inherited polyposis syndromes: familial adenomatous polyposis, MUTYH-associated polyposis, generalized juvenile polyposis syndrome, Peutz-Jeghers syndrome, and Cowden syndrome. Sporadic FGPs are usually innocuous but syndromic FGPs can progress to dysplasia and gastric adenocarcinoma. We describe a new autosomal dominant syndrome characterized by fundic gland polyposis and gastric cancer in a large Australian family and in two smaller North American families. We refer to the syndrome as Gastric Adenocarcinoma and Proximal Polyposis of the Stomach (GAPPS). The disorder exhibits variable expression and incomplete penetrance coupled with a significant risk of gastric cancer that is masked by the polyposis. Analysis of the genes known to cause gastric polyposis was normal. The key clinical features of this disorder are gastric polyposis with dysplastic fundic gland polyps, exclusive involvement of the gastric body and fundus, an inverse association with current $H$. pylori infection, sparing of the remainder of the gastrointestinal tract, and autosomal dominant mode of inheritance. At-risk family members require regular endoscopic surveillance and, depending on physical maturity and degree of polyposis, prophylactic gastrectomy. Acknowledgements: Support from the NHMRC. We thank Dr J Beesley for his assistance, the clinical genetics services and pathology laboratories of Western Australia, Queensland and South Australia and, most importantly, the members of these families.

\section{METABOLIC AND MONOGENIC CAUSES OF SEIZURES IN NEONATES AND YOUNG INFANTS}

\section{J.L. K.Van Hove}

Pediatrics/Clinical Genetics and Metabolism, University of Colorado, United States

Seizures in neonates and young infants present a frequent diagnostic challenge. After exclusion of acquired causes, disturbances of the internal homeostasis and brain malformations, the physician must evaluate for inborn errors of metabolism and for other non-malformative genetic disorders as the cause of seizures. The metabolic causes can be categorized into disorders of neurotransmitter metabolism, disorders of energy production, and synthetic or catabolic disorders associated with brain formation, dysfunction, and degeneration. Other genetic conditions involve channelopathies, and disorders resulting in abnormal growth, differentiation and formation of neuronal populations. These conditions are important given their potential for treatment and the risk for recurrence in the family. This presentation will succinctly review the meta- bolic and genetic non-malformative causes of seizures in neonates and infants less than 6 months of age. Differential diagnostic clues, radiological examples, and a practical paradigm for evaluation will be given.

\section{ANABOLISM: PRACTICAL STRATEGIES}

\section{J. L. K.Van Hove}

Pediatrics/Clinical Genetics and Metabolism, University of Colorado, Aurora, United States

To maintain anabolism, the body needs sufficient calories, and essential building blocks such as essential amino acids, essential fatty acids, vitamins, and minerals. The source of the calories depends on the availability with during fasting a switch from carbohydrate use to glycogenolysis, protein and fat catabolism with accompanying ketogenesis. Fat breakdown occurs between 15 and 20 hours fasting. Prevention of fat catabolism requires avoidance of fasting for longer than this duration. Practical issues arise around night time fasting. If short term catabolism has to be prevented during illness, then a solution based on alpha-dextrin maltose can be used. Solutions of increasing concentration with age given in frequent small amounts are best tolerated. Alternatives can be simple sugar based or combinations with fat included. If intravenous nutrition is required, glucose $10 \%$ solution at 1.5 times maintenance provides part caloric support, but for sufficient caloric intake lipid at $2 \mathrm{~g} / \mathrm{kg} /$ day or more should be added. Early introduction of protein will result in more rapid protein synthesis, even during episodes of hyperammonemia. To limit protein, an intravenous solution of essential amino acids in combination with a regular protein source is used being careful to avoid amino acid imbalances. Tight glucose control with early use of insulin (glucose $<170 \mathrm{mg} / \mathrm{dL}$ ) further improves anabolism. Combined use of these anabolic factors together with hemodialysis and intravenous ammonia scavenging medications has allowed control of ammonia in 12 hours without rebound. Brain MRI scan at 3 days later reviewed using a new scoring system then allows for recognition of neurological sequelae to guide further management. Finally, similar principles can be applied for intravenous ketogenic diet, but pancreatitis is a concern. The main factors now include immediate introduction of intravenous lipid, immediate start of essential amino acid supplementation in hyperammonemia, early use of insulin, all aiming at immediately supporting anabolism at optimal levels.

\section{USING MOUSE MODELS TO UNDERSTAND THE DEVELOPMENTAL BASIS OF CILIOPATHIES \\ C.Wicking',A.Ashe', N. C. Butterfield², L. Town², A. Courtney²,V. Metzis², \\ A. Cooper ${ }^{2}$, R. Barry'2, F. Olsson ${ }^{2}$, B. J.Wainwright ${ }^{2}$, E.Whitelaw' \\ ${ }^{1}$ Institute for Molecular Bioscience, University of Queensland, Australia \\ ${ }^{2}$ Epigenetics Laboratory, Queensland Institute for Medical Research, Australia}

Ciliopathies constitute an ever-expanding group of pleiotropic disorders caused by dysfunction of the primary cilium, a solitary finger-like projection that plays a fundamental role in cellular signaling. Ciliopathies are characterized by an overlapping set of phenotypes including polydactyly, kidney cysts, retinal degeneration and chondrodysplasia, as well as more common phenotypes such as diabetes and obesity. Craniofacial anomalies have more recently been recognized as common features of ciliopathies. Many of the phenotypes associated with ciliopathies suggest a defect in hedgehog signaling, and the primary cilium has been shown to be an essential mediator of this pathway. We have identified a mouse mutant with a defect in the cellular trafficking machinery responsible for assembly of the primary cilium, and show that in a number of contexts hedgehog signaling is altered. By comparing the embryonic phenotypes of this mutant with mouse mutants of the hedgehog receptor patched, we can begin to dissect the contribution of hedgehog signaling to the phenotypes underlying ciliopathies, and to understand the mechanistic basis for some of these phenotypes. A thorough characterization of phenotypes in animal models of cilia dysfunction has the potential to aid in the future characterization of undefined syndromes as ciliopathies. 


\section{FKBP5: AN HPA-AXIS GENE IMPLICATED IN MULTIPLE PSYCHIATRIC PHENOTYPES}

V. L.Willour', A. M. Stütz ${ }^{2}$, R. S. Lee',Y. Huo ${ }^{2}$, P. P. Zandi ${ }^{4}$,J. B. Potash'

${ }^{1}$ Department of Psychiatry and Behavioral Sciences, Johns Hopkins University, United States

${ }^{2}$ Johns Hopkins School of Medicine, United States

${ }^{3}$ European Molecular Biology Laboratory, Australia

${ }^{4}$ Department of Genome Biology, Department of Mental Health, Germany

${ }^{5}$ Johns Hopkins Bloomberg School of Public Health, United States

The FKBP5 gene product forms part of a complex with the glucocorticoid receptor and can modulate cortisol-binding affinity. As such, it plays a key role in the regulation of the hypothalamic-pituitary-adrenal (HPA) axis and the body's stress response. Variations in the gene have been associated with increased risk for multiple psychiatric phenotypes, such as post-traumatic stress disorder and mood disorders. In our initial FKBP5 study, we identified multiple SNPs with evidence for association in a bipolar disorder sample set, with the strongest evidence for association localizing to rs4713902 $(p=.0001)$. Next, we subjected mice to chronic glucocorticoid treatment and characterized the resulting changes in Fkbp5 methylation levels (7\% decrease in methylation in the hippocampus; $p=.004)$ and expression levels (30\% increase in expression in the hippocampus; $p=.0063$ ) in the treated mice. Now, we have resequenced intronic hormone response elements that regulate FKBP5 expression and have genotyped the newly identified and potentially functional variants in three bipolar disorder sample sets. Further genetic, expression, and epigenetic studies such as these should help clarify the role of FKBP5 in psychiatric phenotypes.

\section{PSYCHIATRIC GENETICS IN THE 21ST CENTURY}

\section{V.Willour}

Johns Hopkins University, United States

At the end of the 20th century, psychiatric geneticists found themselves struggling with the same challenges faced by geneticists studying other complex phenotypes, including sample sets with insufficient power to detect risk loci of small effect and technological limitations that prevented comprehensive assessment of genetic variation throughout the genome. The situation was further complicated by the phenotypic heterogeneity present in psychiatric disorders. During the past decade, the field of psychiatric genetics has taken several complementary approaches to address these challenges. First, the field has made an effort to develop an atmosphere that emphasizes the importance of collaboration. The best example of this is the Psychiatric GWAS Consortium (PGC), an international collaborative effort that was formed with the goal of conducting meta-analyses on genome-wide association study (GWAS) datasets for schizophrenia, bipolar disorder, major depression, autism, and ADHD. These large-scale genetic analyses will provide an unprecedented opportunity (with unprecedented power) for the study of psychiatric phenotypes. Second, the field has started to rethink phenotypic classifications, leading to the incorporation of subphenotypes and cross-disorder analyses into study designs. Third, the field is developing a good track record in the successful utilization of high-throughput technologies, leading to significant advances such as the identification of genome-wide significant GWAS findings in schizophrenia and bipolar disorder. Noteworthy progress has also been made in other more poorly understood psychiatric phenotypes, including suicidal behavior. While the field of psychiatric genetics still faces the technical and scientific challenges inherent to the study of all complex genetic phenotypes, the 21 st century holds the promise of significant advances towards the understanding of these complicated and very human phenotypes.

\section{KABUKI SYNDROME: SURPRISING INSIGHTS FROM} CLINICAL AND MOLECULAR STUDIES

\section{B. Wollnik}

Institute of Human Genetics and Center for Molecular Medicine Cologne,

University of Cologne, Germany

Epigenetic control of developmental processes is a fascinating mechanism by which spatial and temporal expression of distinct genes and pathways is regulated. Alterations of epigenetic mechanisms have been mainly associated with the pathogenesis of cancer, but very recently also with the occurrence of congenital malformation syndromes. An up-to-date example is the identification of de novo dominant mutations in the MLL2 gene using a whole-exome sequencing strategy in patients with Kabuki syndrome. Kabuki syndrome is one of the classical, clinically well-known multiple congenital anomalies/mental retardation syndromes, mainly characterized by a very characteristic facial appearance in addition to e. g. developmental delay, short stature, persistent fingerpads, and urogenital tract anomalies. In our study, we sequenced all 54 coding exons of the MLL2 gene in a large cohort of patients with Kabuki syndrome. We identified mainly de novo mutations in almost $60 \%$ of the cases. The mutations were located throughout the MLL2 gene without a clustering in specific exons of the gene or domains of the protein. Interestingly, genotype-phenotype comparison indicated a higher frequency of specific symptoms in MLL2-positive cases. We developed innovative candidate gene approaches and combine these with whole-exome sequencing in parent-patient trios to identify additional genes for Kabuki syndrome. Present findings will be presented.

\section{WHOLE-EXOME SEQUENCING APPROACHES IN RARE DISEASES: NEW CONCEPTS, NEW GENES, AND FASCINATING FINDINGS}

\section{B.Wollnik}

Institute of Human Genetics and Center for Molecular Medicine Cologne,

University of Cologne, Germany

Novel sequencing technologies as well as adopted conceptual strategies can dramatically speed up gene identification in medical genetics. There was little doubt that massive parallel sequencing would have a great impact on studying causative genes for Mendelian disorders in the future, but the last months have impressively shown that this future has already started. We are now expecting a big wave of gene identification studies using these novel sequencing technologies. It is important to note that only together with subsequent functional work on identified proteins and pathways these novel technologies will elucidate underlying pathogenic mechanisms. This talk will present our own recent experiences in using whole-exome-based approaches in medical genetics and show successful examples, which shed light into the pathogenesis of selected rare monogenic disorders.

NOVEL INSIGHTS INTO THE MOLECULAR PATHOGENESIS OF HUMAN LIMB MALFORMATION SYNDROMES

\section{B.Wollnik}

Institute of Human Genetics and Center for Molecular Medicine Cologne,

University of Cologne, Germany

Congenital limb malformations can occur isolated or in combination with other organ manifestations,for example, craniofacial anomalies, in a variety of known malformation syndromes. A number of different phenotypes inherited in Mendelian disorders have been mapped over the last years and causative genes have been identified. Together with the subsequent functional analysis of identified genes and mutations in vitro or in model organisms, these findings gave fascinating insights into the physiological and pathophysiological pathways of limb development. Several structures, pathways, and genetic key players have been identified that control limb development, for example, the apical ectodermal ridge (AER) that directs the proximo-distal outgrowth from the tip of the limb bud, which is dependent on fibroblast growth factor (FGF) signaling or the zone of polarizing activity (ZPA) controling the anterior-posterior patterning (ZPA). Combining classical mapping approaches and the recent fundamental advances in sequencing technologies and its applications, such as whole-exome sequencing, the power of gene identification studies was dramatically increased. This talk will present own data on different monogenic limb malformation syndromes, which gave fascinating new insights into limb development. 


\section{Submitted Abstracts}

\section{NUTRITION SUPPORT AND GROWTH OF TWO PATIENTS WITH CARNITINE-ACYLCARNITINE TRANSLOCASE DEFICIENCY}

R. M. D.Akroyd, E. E. Glamuzina, C. J.Wilson

Starship Children's Health, Auckland City Hospital, Auckland, New Zealand

Carnitine-acylcarnitine translocase (CACT) deficiency is a rare, often severe disorder of long chain fatty acid $\beta$-oxidation. The diagnosis can be suggested on expanded newborn screening (E-NBS) but frequently patients have already presented. The principles of dietetic management are maintenance of adequate growth with MCT based feeds and inhibition of endogenous lipolysis. Two unrelated males presented in the first 24 hours of life with severe hypoglycemic encephalopathy, rhabdomyolysis and variable degrees of hypertrophic cardiomyopathy, complicated by arrhythmia in Case 2 . CACT deficiency was suspected from elevated palmitoylcarnitine on E-NBS and was later molecularly proven. All symptoms significantly improved and cardiomyopathy resolved with commencement of high carbohydrate intravenous fluids and MCT feeds. Case 1, initially NG fed, established full oral feeding by 2 months of age. His clinical course was complicated by recurrent episodes of metabolic decompensation. A gastroenteritis illness at 9 months resulted in significant feeding regression. Subsequently he required $119 \%$ of estimated energy requirements (EER) via NGT and later gastrostomy for normal growth and metabolic stability. Now at 42 months of age he requires $104 \%$ of EER for growth. Weight is tracking the 50th-75th percentile. He is mildly delayed across all developmental indices. He continues to have significant oral aversion and remains fully enterally fed. Case 2 was feeding orally by 3 weeks of age. At 6 months this partially regressed following a minor illness, requiring gastrostomy insertion at 8 months for overnight and top-up feeds. Now at 16 months of age he requires $100 \%$ of EER for growth. Weight is tracking the 50th-75th percentile. He has had no further significant decompensations and is only mildly developmentally delayed. These cases demonstrate the effectiveness of early treatment of neonatal onset CACT deficiency but also the need for subsequent close monitoring of growth and feeding in order to maintain adequate metabolic control.

\section{LOW LEVEL METASTATIC GASTRIC CARCINOMA DETECTED IN BONE MARROW BY CYTOGENETIC AND ARRAY ANALYSIS OF CELLS DEMONSTRATING FGFR2 AMPLIFICATION.}

J.Anderson', R. Brookwell', B. Lundie', E. Jones', J. Harraway', H. Wordsworth', S. Bleakley ${ }^{2}$

${ }^{1}$ Cytogenetics, Sullivan Nicolaides Pathology, Brisbane, Australia

${ }^{2}$ John Flynn Hospital, Tugun, Australia

A 76-year-old male referred for chromosome analysis of chronic lymphocytic leukemia showed a small number of metaphase cells containing multiple chromosome abnormalities and double minutes. The abnormal population could not be confirmed by FISH, and was negative for MYC, MLL and MDM2 amplification. An array was performed which showed increased copy number at the FGFR2 locus. A FISH probe for FGFR2 was applied, and confirmed that the double minutes contained copies of FGFR2. It was later established that the patient was also suffering from an aggressive gastric cancer, so this is likely to be the source of the abnormal FGFR2 amplified cells, although these were not evident morphologically. FGFR2 amplification is known to be associated with gastric cancers, mainly through work with cell lines, rather than directly from tumors. This case demonstrates that an array can detect amplification of specific gene sequences in very small cell populations.

LOSS OF HETEROZYGOSITY (LOH) ANALYSES IMPLICATES PROTEIN TYROSINE PHOSPHATASE TYPE J (PTPRJ) IN NHL LYMPHOMAGENESIS

C.A.Aya-Bonilla', M. Green ',2, E. Camilleri', P. Marlton ${ }^{3}$, R.A. Lea', M. Gandhi ${ }^{4}$, L. R. Griffiths'

${ }^{1}$ Genomics Research Centre, Griffith University, Gold Coast, Australia

${ }^{2}$ Dana Faber Cancer Institute, Harvard Medical School, Boston, United States

${ }^{3}$ Princess Alexandra Hospital, Queensland Health, Brisbane, Australia

${ }^{4}$ Clinical Immunohaematology Laboratory, Queensland Institute of Medical Research, Brisbane, Australia

Non-Hodgkin's lymphoma (NHL) is the most common type of lymphoma. Nevertheless, its etiology and pathogenesis remain unclear because of heterogeneity in biology, genetics and chemoresponse. The recent implementation of Hidden Markov Model (HMM) and Cohort Heterozygosity Comparison (CHC) algorithms in LOH analysis of SNP array data has allowed the identification of novel TSGs that potentially can be involved in oncogenesis, when germ-line DNA from patient is unavailable. Here, we employed high-density SNP array analysis of tumors from the two of the most frequent subtypes of NHL, follicular lymphoma (FL) and diffuse large B-cell lymphoma (DLBCL). This revealed a high frequency of LOH over the chromosomal region of $11 \mathrm{p} 11.2$, containing the gene encoding the receptor-type protein tyrosine phosphatase, PTPRJ. LOH of this gene has been previously described in colorectal, lung, thyroid and breast cancer and its gene product regulates key survival pathways in B-cells, such as B-cell receptor signaling and nuclear factor kappa-B signaling. We therefore validated LOH of the PTPRJ region by microsatellite analysis of an extended cohort of NHL tumors and found significantly increased rates of homozygosity compared to a patient-matched control population. Furthermore, sequencing of homozygous cases revealed a strong association between $\mathrm{LOH}$ and the presence of a non-synonymous SNP in the PTPRJ coding region. Together, these results show the utility of LOH screening in identifying novel tumor suppressor genes, and implicate LOH of the PTPRJ gene as a potential lymphomagenic mechanism.

TWO DE NOVO MUTATIONS AT THE SAME NUCLEOTIDE IN THE OTC GENE OF A MALE

B. Bennetts ${ }^{1,3,4}$, K. Fisk $^{1,3}$, K. Bhattacharya ${ }^{2,3}$

${ }^{1}$ Department Molecular Genetics, The Children's Hospital at Westmead, Sydney, Australia

${ }^{2}$ Department of Biochemical Genetics, The Children's Hospital at Westmead, Sydney, Australia

${ }^{3}$ Discipline of Paediatrics and Child Health, The University of Sydney, Australia

${ }^{4}$ Discipline of Genetic Medicine, The University of Sydney, Australia

Background: Ornithine Transcarbamylase (OTC) deficiency is a heterogeneous $\mathrm{X}$-linked disorder comprising severe neonatal-onset hyperammonemic encephalopathy and later-onset disorders including headaches, personality changes and acute collapse. Case Presentation: A developmentally normal 18 -month-old boy presented with seizures, acute encephalopathy and elevated ammonia $115 \mathrm{~mol} / \mathrm{L}(10-50)$ during recovery. CSF glutamine was $1085 \mathrm{~mol} / \mathrm{L}$ (319-742) and arginine was $7 \mathrm{~mol} / \mathrm{L}(12-30)$ with increased urinary orotate. His mother had a normal phenotype. Method: DNA was extracted from two blood specimens and a urine specimen from the proband. In addition, DNA was also extracted from the proband's mother. Initially the coding region and the intron-exon boundaries of the OTC gene (NM_000531.5) were sequenced and analyzed. In subsequent analyses 
only exon 6 was sequenced. The proband had a karyotype to exclude Klinefelters syndrome. Results: Sequence analysis revealed two mutations at the same nucleotide: c.542A $>\mathrm{T}$ (p.Glu181Val) and c.542A $>\mathrm{G}$ (p.Glu181Gly). Sequencing indicated the c.542A $>\mathrm{T}$ was $2-3$ times more prevalent than the $c .542 \mathrm{~A}>\mathrm{G}$. This result was reproduced with the second blood specimen and DNA from urine. Neither mutation was present in the mother. The proband's karyotype was normal (46XY) excluding this result being due in part to Klinefelters syndrome. Discussion: The presence of mutations at residue 181 is consistent with OTC deficiency. The minor allele p.Glu181Gly has been reported in a neonate. The major allele p.Glu181Val is novel and is predicted by PolyPhen- 2 to be benign though SIFT predicts it to affect protein function with low confidence. In addition, these variants may affect splicing and predicted splicing alterations will be discussed. The origin of the two variants is puzzling. Possible models include a de novo mutation in the mother's X chromosome followed by a somatic mutation in the fetus, or an initial somatic mutation in the fetus, followed by a subsequent second mutation and loss of the original maternal allele.

\section{A PHENOME SCAN FOR HERITABLE TRAITS ASSOCIATED WITH METABOLIC SYNDROME IN THE GENETIC ISOLATE OF NORFOLK ISLAND}

M. Benton', R. Lea', H. Cox', D. Macartney-Coxson'², D. Eccles ${ }^{2,3}$, M. Hanna', G. Chambers ${ }^{3}$, L. Griffiths'

${ }^{1}$ Genomics Research Centre, Griffith University, Australia

${ }^{2}$ Institute of Environmental Science and Research, New Zealand

${ }^{3}$ School of Biological Science, Victoria University of Wellington, New Zealand

Background: Metabolic syndrome (MetS) is a clustering of common disorders (e.g., obesity, insulin resistance, hypertension and high cholesterol). MetS greatly increases risk of type-2 diabetes (T2D) and cardiovascular disease (CVD) and prevalence is on the increase in Australia. The onset and severity of MetS is influenced by a combination of environmental factors (i.e., high fat/sugar diet, smoking, physical inactivity) and genetically heritable modifiers. Understanding the 'envirogenomic' risk profile of MetS should help personalize the prevention and treatment of this growing public health issue. The use of genetic isolates can facilitate the identification of envirogenomic risk factors of disease because of reduced genetic and environmental diversity. Aims: To determine the prevalence of clinical MetS in the isolated population of Norfolk Island (NI), and conduct statistical genetic analysis to identify composite MetS phenotypes with high genetic heritability - a phenome scan — for subsequent molecular genetic studies. Methods: Clinically defined MetS was determined using the 'Harmonised' criteria. An unsupervized Principle Components Analysis (PCA) was performed on 37 biochemical and anthropometric measures to identify new MetS-related phenotypes. The genetic analysis program 'SOLAR' was then utilized to estimate the heritability of these phenotypes in the NI pedigree. Results: Prevalence of clinically-defined MetS in the NI population was $26.4 \%$ compared to $22 \%$ in mainland Australia. Although the clinical MetS phenotype was not found to be heritable $(P>.05)$, PCA of 37 traits extracted 13 composite phenotypes which explained $>75 \%$ of the total variance and yielded statistically significant heritability estimates ranging from 0.22 0.54 . The most heritable component was loaded with 6 measures related to kidney dysfunction - including blood pressure, creatinine, urea, uric acid. Conclusions: This study showed a high prevalence of MetS in the NI isolate and a phenome scan identified a novel renal-linked MetS phenotype with high heritability. This MetS phenotype in particular warrants further investigation in terms of identifying genomic risk profiles and interactions with environmental factors in the NI population.
PRENATAL GENETIC COUNSELING FOR SINGLE GENE DISORDERS: COMPLEX AND CRUCIAL

J. Berkman, K. Chenoweth, P. McGrath

Genetic Health Queensland, Royal Brisbane and Women's Hospital, Herston, Australia

The Maternal Fetal Medicine (MFM) service at the Centre for Advanced Prenatal Care at the Royal Brisbane and Women's Hospital (RBWH) in Brisbane, Queensland offers state of the art services including fetal ultrasound, MRI and fetal therapy and surgery. The MFM service also offers numerous diagnostic procedures including chorionic villus sampling (CVS) and amniocentesis, followed by gene and/or chromosome testing. Genetic counseling forms an integral part of the MFM service and is crucial in cases of fetal abnormality and where prenatal diagnosis is required. We present here an overview of the genetic counseling caseload at the RBWH over a one year period, with emphasis on the conditions for which fetal testing for single gene disorders was performed. The role of the genetic counselor in facilitating fetal testing, promoting patient understanding and ensuring appropriate support and follow up is discussed. Case examples which highlight the complex issues faced by both the client and the genetic counselor will be presented.

\section{VARIANT EVI1 REARRANGEMENTS IN MYELOID \\ DISORDERS}

M. Bhuimbar, J.Anderson, R. Brookwell, P. Sutton-Davies, H.Wordsworth

Cytogenetics, Sullivan Nicolaides Pathology, Brisbane, Australia

Chromosome rearrangements and their resultant gene disruptions have long been recognized as a major mechanism contributing to leukemic pathogenesis. Recurring rearrangements of $3 q$ involving 3q26.2 (EVI1) have been reported in cases of myelodysplastic syndrome (MDS), AML and blast crisis CML. Most patients with EVI1 rearrangements (also known as 3q syndrome) present with increased blast counts, normal to elevated platelet levels, increased megakaryocytes and marked trilineage dysplasia. EVI1 rearrangements such as $\operatorname{inv}(3)(\mathrm{q} 21 \mathrm{q} 26.2)$ and $\mathrm{t}(3 ; 3)(\mathrm{q} 21 ; \mathrm{q} 26.2)$ have been reported in the literature as occurring in $1-2 \%$ of cytogenetically abnormal AML . Our laboratory reviewed a total of eight cases over a 2-year period and found that most cases evolved to AML from a previously diagnosed MDS. FISH studies using the Poseidon EVI dual-color break apart probe showed some patients to have a variant signal pattern due to a degree of heterogeneity within the EVI1 gene, although this seemed to have little if any effect on the prognostic implications of sometimes cryptic $3 q$ rearrangements. The prognosis of patients with EVI1 rearrangements is generally considered to be poor, EVI1 overexpression causing a poor response to chemotherapy. We describe our experience of eight cases of myeloid disorders with EVI1, using hematological, cytogenetic and FISH findings.

\section{AN EXPLORATION OF GENETIC HEALTH PROFESSIONALS' EXPERIENCE WITH DIRECT TO CONSUMER GENETIC TESTING IN THEIR CLINICAL PRACTICE}
G. R. Brett ${ }^{1,2}$, S.A. Metcalfe ${ }^{1,2}$, D. J.Amor ${ }^{2,3}$, J. L. Halliday ${ }^{1,2}$
${ }^{1}$ Public Health Genetics, Murdoch Childrens Research Institute, Melbourne, Australia
${ }^{2}$ Department of Paediatrics, The University of Melbourne, Australia
${ }^{3}$ Genetic Health Services Victoria, Murdoch Childrens Research Institute, Melbourne, Australia

Background: Direct to consumer genetic testing (DTC-GT) allows individuals to obtain genetic tests and whole genome scans directly from private companies. In many cases, these tests have unknown clinical utility and therefore their use to assess disease risk is controversial. In addition, the personalized genetic risk information provided may be interpreted solely by consumers without guidance from health professionals. Such measures can inappropriately heighten health related anxiety or apathy and subsequently lead to poor life choices. There is also potential for other psychological effects on consumers due to lack of knowledge and support. Methods: This study is a survey of genetic counselors, clinical geneticists and genetic nurses 
using an online questionnaire regarding their experiences with consumers who have undertaken DTC-GT. Participant recruitment is via emailed invitations to members of the Human Genetics Society of Australasia (HGSA) and British Society for Human Genetics (BSHG). Results: From 158 survey respondents in the first two weeks, 117 were qualified in the selected genetic health professions of whom $8.5 \%$ reported clients presenting after undertaking DTC-GT. Preliminary data indicate the majority of clients are self referred or referred by primary carers. They typically sought DTC-GT for identification of monogenic conditions, including carrier testing, or susceptibility/predisposition for complex conditions. Survey participants reported a range in clients' level of satisfaction with their chosen DTC-GT, although few appeared to regret testing. Participants also had mixed views on the benefits of post-DTC-GT consultation, ease of managing such cases and clinical significance of DTC-GT. Importance: The overall impact of DTC-GT on consumers, both physically and psychologically, is not yet understood. Identification of the present role genetic health professionals play in DTC-GT will allow preparation of appropriate guidelines and therefore better management of future cases. Further studies may then focus on the uptake of DTC-GT within the general population.

\section{GENETIC PROFILING OF ENDOMETRIOSIS}

T. Buchholz, C.Adelfalk

Gyn Gen Lehel, Muenchen, Germany

Endometriosis is a clinically unspecific disease, where diagnosis is often made after years of suffering following invasive procedures. Despite several theories, the cause is unknown and a multiple genetic contribution is very likely. Different genes implicated in the genesis of endometriosis have been suggested and investigated in healthy and endometriosis patients. Two polymorphic genes associated with an increased risk of developing endometriosis are E-cadherin and the progesterone receptor. ${ }^{1,2,3}$ Our aim is to use such genes as reproducible genetic biomarkers in the diagnostics of patients prior to invasive diagnostics. Up to now we have collected a series of 10 patients, which fulfil the classical criteria of endometriosis type 2 . The genetic testing set up has been completed and the first patients have been analyzed. A 3 '-UTR C->T polymorphism in the E-cadherin gene is analyzed by PCR and subsequent enzymatic cleavage. ${ }^{2}$ The progesterone receptor polymorphism (PROGINS) of a 306-base pair insertion in introm $\mathrm{G}$ is detected by use of PCR, resulting in a longer product for the polymorphism..$^{1,3}$ A genotypephenotype correlation will be established following the completion of the genetic testing and more patients are anticipated in an extended study. The correlation beween various stages of endometriosis and the genotype of the two genes analyzed will provide a useful tool in identifying endometriosis risk patients prior to invasive procedures.

${ }^{1}$ Gynecological Endocrinology 2007; 23:29-33.

${ }^{2}$ Reproduction 2007; 134:373-378.

${ }^{3}$ Fertility and Sterility 2002; 77:309-312.

\section{PROMOTER POLYMORPHISMS IN THE FOXP1 GENE AND RISK OF NON-HODGKIN LYMPHOMA: A PILOT STUDY}

\section{E. Camilleri, C.Aya-Bonilla, L. Griffiths}

Genomics Research Centre, Griffith University, Southport, Australia

The identification of susceptibility markers for non-Hodgkin's lymphoma (NHL) is critical as some NHL subtypes have an aggressive disease progression. FOXP1 is a member of the forkhead box/winged-helix domain-containing family of transcription factors. The specific function of FoxP1 transcription factor remains unclear; however, the overexpression of FOXP1 in NHL subtypes clearly indicates a role for FOXP1 in lymphoma pathogenesis. FOXP1 is commonly targeted by translocations and copy number variations in NHL; however, FOXP1 is more frequently overexpressed independently of such genomic aberrations. An alternative mechanism that may cause the deregulated expression of FOXP1, are single nucleotide polymorphisms (SNP) within the promoter region. These polymorphisms may disrupt essential transcription factor binding sites, which could affect gene expression. This study aims to ascertain whether FOXP1 promoter polymorphisms confer susceptibility to NHL. This study investigated three SNPs (rs13096388, rs830600 and rs1499893) within the promoter region of the FOXP1 gene, in a pilot cohort of 136 Australian Caucasian NHL patients and 96 matched controls. The alleles were detected using PCR followed by restriction fragment length analysis and was visualized by agarose gel electrophoresis and UV transillumination. The allele frequency for rs13096388 controls was $\mathrm{C}=0.425, \mathrm{~T}=0.575$ and cases $\mathrm{C}=0.299, \mathrm{~T}$ $=0.701 ; \mathrm{rs} 830600$ controls was $\mathrm{T}=0.783, \mathrm{C}=0.217$ and cases $\mathrm{T}=$ $0.496, \mathrm{C}=0.504$; and $\mathrm{rs} 130966388$ control samples all had the $\mathrm{G}$ allele and only 1 case had the alternative $\mathrm{C}$ allele. The overexpression of FOXP1 transcription factor is prognostic of NHL patient outcome, however, the mechanism causing its deregulation is unknown. The allele frequencies for both rs 13096388 and rs830600 SNPs vary greatly between NHL cases and controls, with high frequencies of the minor allele in cases. This trend suggests that SNPs with the promoter region of the FOXP1 gene confer susceptibility to NHL.

\section{A GENETIC APPROACH TO INVESTIGATE CLINICAL AND PSYCHOLOGICAL OUTCOMES FOR WOMEN WITH BREAST CANCER - ESTABLISHMENT OF A GRIFFITH HEALTH INSTITUTE BREAST CANCER BIOBANK}

D. F. Chacon Cortes', S. Chambers ${ }^{2,3}$, P.Youl ${ }^{3}$, R.A. Smith', R. Lea', L. R. Griffiths'

1 Genomics Research Centre, Griffith Health Institute, Griffith University, Southport, Australia

2 Behavioral Basis of Health, Griffith Health Institute, Griffith University, Southport, Australia

3 Cancer Council Queensland, Australia

Breast cancer is the most common cancer affecting Australian women. As the population increases and ages the number of women diagnosed is expected to increase, representing a significant burden on health and health-related services. Breast cancer is believed to arise from the accumulation of genetic mutations of genes involved in critical pathways such as proliferation, apoptosis, and repair of DNA. Most of these mutations occur in somatic tissue and are not inherited; however, 5-10\% of cases are the result of inherited mutations. A number of studies have also implicated a variety of susceptibility genes as playing a role in breast cancer risk. In addition, gene expression studies have implicated a number of genes involved in prognosis and progression of this particular disease. In collaboration with the Cancer Council Queensland (CCQ), this study aims to create a DNA and RNA Biobank from collected breast cancer patients for studies of genetic susceptibility and gene expression variation in relation to disease development, prognosis and treatment response. Comprehensive demographic, lifestyle, social, environmental and clinical data from 3300 newly diagnosed breast cancer cases will be collected along with blood samples to establish a DNA and RNA bank located at the Genomics Research Centre at Griffith University, to enable future genetic susceptibility and gene expression studies. This study will use a multi-level approach to link epidemiological and biomolecular factors associated with breast cancer susceptibility, diagnosis, prognosis and treatment response allowing the continued improvement or the development of new public health programs and services to reduce the burden of breast cancer on Australian women and their families.

\section{HOMOCYSTEINE AND ERYTHROCYTE SEDIMENTATION RATE CORRELATE WITH CEREBROVASCULAR DISEASE IN FABRY DISEASE}

R. Cheung, D. O. Sillence, M. C.Tchan

Genetic Medicine, Westmead Hospital, Westmead, Australia

Background: Cerebrovascular disease (CVD) is a common clinical problem in Fabry disease, however expression of this disease mani- 
festation is not uniform and risk factors for its development are not well studied. Aim: To evaluate associations between numerous traditional risk factors and CVD in patients with Fabry disease. Methods and Results: 32 Fabry disease patients (14 male) were studied, with 15 having a positive history of CVD. Student $t$ tests were used to compare the positive and negative CVD groups and logistic regression was used to look for correlations with CVD history. CVD positive patients were older $(49.73$ v 37.59 years, $p<.001)$ and had worse renal function (glomerular filtration rate (GFR) $61.525 \mathrm{v}$ $96.613 \mathrm{ml} / \mathrm{min} / 1.73 \mathrm{~m} 2, p<.005)$, higher homocysteine $(17.791 \mathrm{v}$ $10.525 \mathrm{~mol} / \mathrm{L}, p<.05$ ), higher erythrocyte sedimentation rate (ESR) $(23.8$ v $7.64 \mathrm{~mm} / \mathrm{hr}, p<.001)$, and elevated Mainz Severity Score Index (MSSI) scores $(23.770 \mathrm{v} 11.790, p<.001)$. Correlations were found between age (OR 1.110), GFR (OR 0.950), homocysteine concentration (OR 1.218), ESR (OR 1.164) and the MSSI (OR $1.185)$ scores with a positive CVD history (All $p<.05)$. Conclusion: Elevated homocysteine and ESR are independent risk factors for CVD in Fabry disease. This finding adds to our ability to predict those patients with Fabry disease who are at a higher risk of developing CVD, and may aid in deciding which patients should have primary CVD prevention therapies.

\section{COMPREHENSIVE MOLECULAR TESTING FOR MITOCHONDRIAL DISORDERS}

B. Chong', S. Pantaleo', D. Thorburn², D. Du Sart'

1 Molecular Genetics, Victorian Clinical Genetics Services Pathology, Melbourne, Australia

2 Mitochondrial Research, Murdoch Childrens Research Institute, Melbourne, Australia

Mitochondrial disorders are due to dysfunction of the mitochondrial respiratory chain and they are caused by mutations in mitochondrial DNA (mtDNA) or in nuclear genes that code for mitochondrial components. Mitochondrial disorders are clinically heterogeneous however; there are a number of well defined mitochondrial syndromes that are characterized by their association with certain clinical features. These include chronic progressive external opthalmoplegia (CPEO), Kearns Sayre Syndrome (KSS), leber hereditary optic neuropathy (LHON), mitochondrial encephalomyopathy with lactic acidosis and stroke-like episodes (MELAS), myoclonic epilepsy with ragged-red fibres (MERRF) and neurogenic weakness with ataxia and retinitis pigmentosa (NARP). All together these represent the most common mitochondrial genetic defects that are routinely screened by diagnostic laboratories. Our laboratory has developed a new Single Nucleotide Primer extension assay using Sequenom technology, to genotype 22 mitochondrial mutations and 3 of the most common European autosomal recessive POLG mutations. The disorders tested include Leigh syndrome, NARP, MELAS, MERRF, LHON, deafness, MIRAS and Alpers. From our pediatric cohort data our new expanded panel has increased the yield of frontline DNA testing from $59 \%$ to $84 \%$. For some patients mutations found were typically found only after identifying an enzyme defect in skeletal muscle. Therefore, the expanded panel could potentially spare many patients from the need for an invasive muscle biopsy. In addition, the panel also includes the common mutations for leber hereditary optic neuropathy (LHON), deafness due to mitochondrial disorder and POLG mutations which are associated with mitochondrial depletion. Therefore, this panel is a more comprehensive screen for mitochondrial disorders.

\section{CHALLENGES OF A LOW PROTEIN DIET IN PATIENT WITH VERY LATE DIAGNOSED ORNITHINE TRANSCARBAMYLASE DEFICIENCY LIVING IN SUPPORTIVE ACCOMMODATION}

E. Clover', M. Netting', D. Bratkovic ${ }^{2}$, J. Fletcher ${ }^{3}$

${ }^{1}$ Clinical Dietetics, Royal Adelaide Hospital, Adelaide, Australia

${ }^{2}$ Metabolic Unit, Children, Youth and Women's Health Service, Adelaide, Australia

${ }^{3}$ Genetics and Molecular Pathology, SA Pathology, Adelaide, Australia

Background: Ornithine transcarbamylase deficiency (OTCD) is a urea cycle disorder resulting in an accumulation of waste nitrogen which is neurotoxic. Presentation can occur at any age. Late-onset diagnosis is common in female carriers and may be precipitated by increased metabolic stress. Treatment of OTCD is a restriction of dietary protein, in addition to medication to utilize alternate pathways for the excretion of waste nitrogen and supplementation of arginine. Aims: We report a case of a very late diagnosed OTCD in an elderly female living in supportive accommodation and review the challenges faced in implementing an appropriate low protein diet. Subject: 87-year-old female, living in a low level of care nursing home, diagnosed with OTCD in 2007 after developing encephalopathy and hyperammonemia post a fractured neck of femur. Methods: The patient required a protein restriction of $0.8-1 \mathrm{~g}$ per $\mathrm{kg}$ and was prescribed sodium benzoate and arginine. Ammonia levels normalized; however, branch chain amino acids were low. A diet diary from the patient's nursing home reflected an over restriction of protein $(0.3-0.4 \mathrm{~g}$ per $\mathrm{kg})$. A meal plan and modified protein diet chart were provided. Subsequent diet diaries showed some increase in dietary protein intake but remained below goal. The patient revealed a reluctance to increase dietary protein due to food preferences and fear of increasing ammonia levels. Further education was provided on achieving adequate protein intake and regular diet diaries were requested from the nursing home. Conclusions: Implementing a restricted protein diet in an elderly nursing home patient with very late diagnosed OTCD is challenging. Inadequate provision of protein occurred due to staff lacking knowledge and being unable to attend clinic appointments. The patient's reluctance to consume protein needed to be addressed with education. Face-to-face education with nursing home staff and the patient would be ideal.

\section{PKU SUPER CLINIC: OUR EXPERIENCE}

\section{T. Collins, B. Martino, J. Knight, A. Martin}

Princess Margaret Hospital for Children, Subiaco, Australia

Purpose: The metabolic outpatient clinic at Princess Margaret Hospital for Children (PMH) involves individual patients and their carers, with a variety of metabolic conditions, being seen simultaneously by a multidisciplinary team. An audit of phenylalanine levels of current classical Phenylketonuria (PKU) patients revealed only $27 \%$ had levels were within the recommended range. It was hypothesized by the metabolic dietitians that a discreet PKU clinic would better serve the patients by providing: patient focus specific to the condition; interactive group sessions; a forum for networking to share meal ideas, recipes and resources; an opportunity for patients to see other people with PKU as well as a more appropriate use of health professional resources in a dietetic-led clinic. Method: Only classical PKU patients were included. 50 patients were invited to two clinics per year each, grouped by patient age. Clinics included 20-minute individual consults with doctor and dietitian followed by an opportunity to socialise with other patients, carers, social worker, liaison nurse and company representatives. Specialized PKU products were available for sampling. Summary of Results: Post-clinic attendance, a re-audit of PKU levels showed no improvement in metabolic control, however, anecdotally, carers were very positive about the new format. To objectively measure carer satisfaction a survey monkey was implemented. This confirmed that carers preferred the new format. Despite no improvement in metabolic control we were satisfied that the benefits warranted continuation of the super clinic. The format of the clinic will continue to be monitored and adjusted based on carer and dietitian feedback.

\section{WHEN ROUTINE GENETIC TESTING REVEALS} MISATTRIBUTED PATERNITY

\section{G. Correnti}

Genetic Health Queensland, Royal Brisbane and Women's Hospital, Herston, Australia

With the rapid advancement of genetic testing, it is becoming more common for routine investigations to reveal unanticipated or unsought information. One such circumstance is when genetic testing raises the 
suspicion of misattributed paternity. This situation has the potential to inflict profound emotional burdens on patients and create complex ethical dilemmas for genetic counselors and geneticists regarding whether or not to disclose the information and to whom. The role of the genetic counselor is crucial in facilitating informed consent and minimizing harm to the patient. Presented here is an overview of the challenges that may arise when genetic testing reveals misattributed paternity. Two recent case examples will be discussed to illustrate varying scenarios in which misattributed paternity was suspected after genetic testing was performed. The different approaches that were taken in regards to disclosure will also be outlined.

\section{CASCADE TESTING FOR HEREDITARY HEMORRHAGIC TELANGIECTASIA — SOME INTERESTING CASE STUDIES}

\author{
K. Cox', L. Sanchez', R. Catford', E. Douglas', E. Haan², K. Friend' \\ ${ }^{1}$ Molecular Genetics Unit, SA Pathology at the Women's and Children's Hospital, \\ Adelaide, Australia \\ ${ }^{2}$ South Australian Clinical Genetics Service, SA Pathology at the Women's and \\ Children's Hospital, Adelaide, Australia
}

Hereditary hemorrhagic telangiectasia (HHT) is an autosomal dominant disorder characterized by aberrant vascular development resulting in multisystemic dysplasia and recurrent hemorrhage. Nosebleeds (epistaxes) are usually the earliest clinical symptom, mucocutaneous telangiectases develop in $\sim 30 \%$ of cases at $<30$ years and anemia and gastrointestinal bleeding occurs later in life. $20 \%$ of individuals develop pulmonary arteriovenous malformations which may lead to hypoxemia and paradoxical embolism. Two causative genes have been identified: Endoglin (ENG) on chromosome 9 and ActivinA Receptor Like Type II (ACVRL1/ALK1) on chromosome 12. Initial mutation analysis in these genes was performed at the Genetic Diagnostic Laboratory of the University of Pennsylvania. On identification of a pathogenic mutation, cascade screening to other family members may be offered 'in house'. Since 2003, a total of 17 samples have been sent away for mutation analysis. 11 have returned with a positive result and cascade testing performed. Of these we have three interesting family studies. Family 1: A mutation, c.511C >T (p.R171X) was identified in ENG. Cascade testing revealed a 26 year old female with no presumed clinical symptoms who carried the familial mutation and a 33 year old male with epistaxes who does not carry the mutation. Family 2: A mutation, c.277C $>$ T (R93X), was identified in ENG. Cascade screening identified a 12 year old female with probable clinical symptoms, who did not carry the familial mutation. Family 3: A mutation, c.806T $>$ G (M269R) was identified in ENG. While taking family history a deceased child, with no known cause of death was revealed. DNA extracted from the Guthrie card of this child identified that she carried the familial mutation. We conclude that cascade testing can be beneficial to the family but can also bring up some unexpected results.

\section{CHALLENGES ENCOUNTERED WHEN PROVIDING GENETIC COUNSELING FOR HEMOPHILIA}

\section{B. Culling, J. R. Pinner \\ Department of Molecular and Clinical Genetics, Royal Prince Alfred Hospital, Sydney, Australia}

At Royal Prince Alfred Hospital the Clinical Genetics unit has a close working relationship with the Haemophilia Treatment Centre. Through this association we provide genetic counseling to men with hemophilia and their extended families. These cases may present challenges either in the counseling issues that arise or in conducting genetic testing. We present four cases which demonstrate some of the challenges we have encountered.

1. The difficulty of arranging prenatal testing when the familial mutation had not been identified at initial presentation.

2. The challenge of interpreting obligate carrier testing when the familial mutation is unknown and family separation makes access to familial information unlikely.
3. The complex issues encountered when counseling a man with hemophilia and hepatitis $\mathrm{C}$.

4. Ensuring a woman who was adopted out of a family is aware that there is a $50 \%$ chance she is a carrier of hemophilia

These cases demonstrate the variety of issues and challenges that can be encountered when providing genetic counseling to families with hemophilia. They also highlight the importance of cooperation and communication between services to ensure that individuals have access to accurate information and appropriate opportunities.

\section{A RAPID METHOD FOR THE QUANTIFICATION OF METHYLMALONIC ACID IN PLASMA USING UPLC-MS/MS}

B. Devanapalli, K. G. Sim,W. C. Ip, K. Carpenter

Biochemical Genetics, The Children's Hospital at Westmead, Sydney, Australia

Introduction: Defects in the metabolic pathway of methylmalonyl $\mathrm{CoA}$ and the cofactor adenosylcobalamin can be detected as methylmalonicaciduria (MMAU), which is one of the most common disorders of organic acid metabolism. Methylmalonic acid (MMA) quantitation is useful for differential diagnosis of elevated propionylcarnitine detected on newborn screening, monitoring MMAU patients and assessment of B12 status in other IEM patients on restricted diet at risk of B12 deficiency. Typical methods involve derivatization with butanolic- $\mathrm{HCl}$, followed by LC-MS/MS analysis with a run time of 10 minutes per sample. We recently developed an improved underivatized method using UPLC-MS/MS with a run time of 2 minutes. Method: An aliquot of plasma is ultrafiltered, combined with deuterated internal standard and acidified with formate. MMA is separated from isomeric succinic acid using a 2.1x50mm BEH C18 $1.7 \mathrm{~m} \mathrm{~m}$ column and detected by electrospray ionization tandem mass spectrometry (Acquity TQD, Waters Corporation). A standard curve was prepared by adding in known concentrations of MMA to plasma from a healthy subject. Results: The method was verified by parallel runs of patients and ERNDIM samples with the previous method. The correlation between the two methods was excellent, $r^{2}=.99, \mathrm{y}=0.9787 \mathrm{x}$ +0.0374 . A $t$ test: paired two sample for means $(n=109)$ showed no significant difference $(p=.016)$. The between run precision $(\mathrm{CV})$ was $14.1 \%$ at $0.15 \mu \mathrm{mol} / \mathrm{L}$ and $3.5 \%$ at $2.19 \mu \mathrm{mol} / \mathrm{L}(n=16)$. The method is linear up to $200 \mathrm{~m} \mathrm{~mol} / \mathrm{L}$. Analysis of stored ERNDIM samples ( $n$ $=12$ ) found results within $2 S D$ of consensus means across a wide range of values.

\section{IDENTIFICATION OF SIALYLTRANSFERASE 8B AS A GENERALIZED SUSCEPTIBILITY GENE FOR PSYCHOTIC AND MOOD DISORDERS ON CHROMOSOME 15025-26}

J.A. Donald' ', E. Z. McAuley ${ }^{2,3}$, A. Scimone ${ }^{2}$, Y.Tiwari $^{2,3,4}$, G. Agahi $^{2}$, B. J. Mowry ${ }^{5,6}$, E. G. Holliday ${ }^{5}$, C. Shannon Weickert ${ }^{2,4,7}$, P. B. Mitchell ${ }^{7,8}$, P. R. Schofield ${ }^{2,3,4}$, J. M. Fullerton $2,3,4$

${ }^{1}$ Department of Biological Sciences, Macquarie University, Australia

${ }^{2}$ Neuroscience Research Australia, Sydney, Australia

${ }^{3}$ School of Medical Sciences, University of New South Wales, Australia

${ }^{4}$ Schizophrenia Research Institute, Sydney, Australia

${ }^{5}$ Queensland Centre for Mental Health Research, Brisbane, Australia

${ }^{6}$ Queensland Brain Institute, University of Queensland, Australia

${ }^{7}$ School of Psychiatry, University of New South Wales, Australia

${ }^{8}$ Black Dog Institute, Prince of Wales Hospital, Sydney, Australia

It has been hypothesized that bipolar disorder and schizophrenia share a large proportion of their genetic risk. However much of the overlapping genetic variance remains unexplained. We previously identified a significant linkage peak on 15q25-26 using a broad clinical phenotype, including bipolar disorder (types I and II), recurrent unipolar disorder and schizoaffective disorder. We will present evidence that sialyltransferase 8B (ST8SIA2), a gene previously implicated in schizophrenia susceptibility in Asian cohorts, is a generalized susceptibility gene for bipolar disorder and schizophrenia. A fine-mapping association study of the 15q25-26 linkage interval was conducted in an Australian bipolar disorder case-control cohort $(n=$ 385). Replication of association of a specific risk haplotype was con- 
ducted in an Australian schizophrenia case-control cohort $(n=256)$, and in GWAS genotype data from the NIMH bipolar disorder $(n=$ $2055)$ and schizophrenia cohorts $(n=2550)$. Nominal single point association was observed with two SNPs in ST8SIA2, and a risk haplotype was identified that is significantly over-represented in cases compared to controls in these cohorts. We have also demonstrated marked developmental regulation of this gene. These findings suggest that the ST8SIA2 gene may render the developing brain more vulnerable to secondary genetic or environmental insults during maturation or adulthood.

\section{MOSAICISM AND MULTIPLE NORMAL ALLELES OF FMR1 CCG REPEAT NUMBER IN MALES}

E. Douglas', R. Catford',A. Correll', K. Cox', D. Schultz², D.White', K. Friend'

1 Diagnostic Molecular Genetics Unit, SA Pathology at the Women's and Children's Hospital, Adelaide, Australia

2 Department of Neurology, Flinders Medical Centre, Adelaide, Australia

3 Child Development Unit, Women's and Children's Hospital, Adelaide, Australia

Fragile X syndrome is the second most common cause of intellectual disability after Trisomy 21 and accounts for approximately half of $\mathrm{X}$-linked intellectual disability cases. ${ }^{1}$ In addition to moderate to severe intellectual disability, features also include macroorchidism, large ears, prominent jaw and a long face. The most common mutation (> 99\%), resulting in Fragile X syndrome is an expansion (> 200 CCG repeat copies) of the trinucleotide CCG repeat within the $5^{\prime}$ untranslated region of the FMR1 gene, Subsequent methylation results in decreased levels of FMRP in the brain. Individuals with intermediate CCG repeat copy alleles (55 to 200) are at risk of developing the neurodegenerative disorder, Fragile $X$ associated tremor/ataxia syndrome (FXTAS). We have recently identified two interesting cases where males have two FMR1 CCG repeat alleles that both fall within the normal range (5-54 CCG repeat copies). Case 1 is a 51 -year-old male $(46, \mathrm{XY})$ who was referred for Spinocerebellar ataxia (SCA) screen, including FXTAS analysis, and identified to carry two alleles of 21 and 29 CCG repeat copies. This result was confirmed with an earlier sample that had been stored in our laboratory, and Southern analysis did not detect an expanded allele. Case 2 is a 9-year-old boy with intellectual disability, obesity and ASD, referred for Fragile X syndrome, PCR analysis at the FMR1 CCG repeat revealed two alleles of 30 and 40 CCG repeat copies. Further investigations including Southern and cytogenetic analyses are currently underway for Case 2 . We have previously identified two cases of mosaicism involving alleles within both the premutation and full mutation ranges, as well as a further case which included a normal sized allele detectable by PCR in addition to premutation and full mutation sized bands on Southern analysis. Results and discussion will be presented.

${ }^{1}$ Genetests - www.ncbi.nlm.nih.gov/sites/Genetests: GeneReviews

\section{MOLECULAR CHARACTERIZATION OF A PROXIMAL INTERSTITIAL DELETION OF 4021.22-023}

R. DuPreez', R. Hope', C. Kennedy', L. Morgan', M.W. Partington², M. Hunter², R. Scott'

1 Cytogenetics, Pathology North, New Lambton Heights, Australia

2 Clinical Genetics, Hunter Genetics, Waratah, Australia

A 35-year-old male with intellectual disability and dysmorphic features was referred for chromosome analysis. GTG banded chromosomes were prepared from blood lymphocytes according to standard procedures. We analyzed 50 metaphases and reported a proximal interstitial deletion of the long arm of chromosome 4 . Karyotype: 46, XY, del(4)(q21.3q23). Four years later a repeat sample was sent for possible further tests. We cryopreserved three vials of blood and stored it at -80 degrees C. Sixteen years later we decided to do the precise mapping of the breakpoints by molecular cytogenetic techniques. DNA was extracted from the blood using precautions to prevent degradation of the DNA. High resolution genomic analysis using oligo-microarray comparative genomic
hybridization(aCGH) confirmed the copy number imbalance i.e. a loss, and also delineated the precise break points to be start $4 \mathrm{q} 21.22$ end $4 \mathrm{q} 23$. The size of the interstitial deletion is start 83,578,154 and end $102,109,657$ i.e. $18.5 \mathrm{Mb}$. Probe positions mapped to Ensembl build 54 (hg18). Although a number of comparable cases have been published there is wide diversity in clinical presentation and with respect to size and location of the deletion. Very few cases of deletion 4 have had molecular characterization.

14012 MICRODELETION: A RETT SYNDROME — LIKE PHENOTYPE

C. J. Ellaway ${ }^{1,2}$, E. Bettella $^{3}$, F. Collins ${ }^{1,2}$, A. Hackett ${ }^{4,5}$, F. McKenzie ${ }^{4,5}$, G. Ho ${ }^{1,2}$, A. Darmanian', G. Peters ${ }^{1,2}$, K. Fagan ${ }^{4}$, J. Chrsitodoulou ${ }^{1,2}$

${ }^{1}$ Western Sydney Genetics Program, Children's Hospital at Westmead, Sydney, Australia

${ }^{2}$ Discipline of Paediatrics and Child Health, Sydney Medical School, University of Sydney, Australia

${ }^{3}$ Department of Pediatrics, University of Padova, Italy

${ }^{4}$ Clinical Genetics, Hunter Genetics, Newcastle, Australia

${ }^{5}$ School of Biomedical Sciences, University of Newcastle, Australia

${ }^{6}$ Pathology North (Hunter), John Hunter Hospital,Newcastle, Australia

Rett syndrome (RTT) is a clinically defined neurodevelopmental disorder almost exclusively affecting girls, and characterized by early normal development followed by regression with loss of speech and acquired motor skills, stereotypic hand movements, slowing of brain growth and seizures. Usually sporadic, RTT is caused by mutations in the X-linked MECP2 gene in approximately $90-95 \%$ of classic cases and $40-60 \%$ of individuals with atypical RTT. Mutations in the CDKL5 gene have been associated with the early-onset seizure variant of RTT and mutations in FOXG1 have been associated with the congenital RTT variant.We report the clinical features and array CGH findings of three atypical RTT patients. The three patients, two female and one male all had intellectual impairment, early onset developmental delay without regression, microcephaly and hypotonia. In addition the females had a seizure disorder and agenesis of the corpus callosum. All three were found to have an interstitial deletion of 14q12. The commonly deleted region identified in the females included the PRKD1 gene but not the FOXG1 gene. In silico analysis of the deleted region shared by the three patients identified two large conserved regions $(>2 \mathrm{~kb}$ ) containing potential regulatory elements. The regions lie between FOXG1 and PRKD1, $620 \mathrm{~kb}$ and $684 \mathrm{~kb}$ downstream of FOXG1. Screening of 32 atypical RTT patients did not identify any pathogenic mutations in PRKD1, a protein kinase involved in extracellular receptor-mediated signal transduction pathways. There is phenotypic overlap between congenital RTT variants with FOXG1 mutations and the clinical presentation of our three patients with this $14 \mathrm{q} 12$ microdeletion, not encompassing the FOXG1 gene. We propose that the primary defect in these patients may be misregulation of the FOXG1 gene rather than a primary abnormality of PRKD1, and suggest that array CGH is a mandatory investigation in patients with a RTT phenotype and have no pathogenic mutation in MECP2, CDKL5 or FOXG1.

\section{USE OF FAMILY COMMUNICATION AND PATIENT REVIEW TOOLS - QUALITY ASSURANCE IN CROSS-SERVICE PROVISION}

A. Engel', K. Tucker², S. Greening ${ }^{3}$, L.Warwick'

${ }^{1}$ Genetics Department, Canberra Hospital, Canberra, Australia

${ }^{2}$ Hereditary Cancer Clinic, Prince of Wales Hospital, Sydney, Australia

${ }^{3}$ Hereditary Cancer Clinic, Wollongong Hospital, Wollongong, Australia

Patient follow-up is an essential element of genetic counseling practice. Supporting patients in informing at-risk relatives of the family mutation status is an important aspect of this patient follow-up. Family communication is key in delivering information to at-risk family members in cancer, cardiac, neurological and other speciality genetics. The use of patient review and family communication tools can assist patients receive appropriate follow-up and can improve the provision of care for all family members. Collaboration between the Genetics 
Service at The Canberra Hospital and the Hereditary Cancer Clinics at Prince of Wales and Wollongong Hospitals has led to the production of a quality review tool for patient follow-up and a family communication tool. These tools were initially developed for use in cancer genetics but have since been deployed in general genetics. The impetus for development has been to foster quality assurance and consistency across services. The use of these tools can benefit supervisors, particularly those working across several services, by improving efficiency and promoting a consistent approach to patient and family follow-up. These tools can also guide and support time-stretched genetic counselors, including junior genetic counselors who are often employed in outreach. Ultimately, the development and use of such tools in a coordinated manner within and between services will benefit individuals and families affected by genetic conditions.

\section{A RARE CASE OF PRENATALLY DETECTED TRISOMY 9 \\ K. Evans', P. Guardala', M. De Papi', E. Gunning',A.Vipond', J. Belton', F. Carmody ${ }^{2}$ \\ ${ }^{1}$ Cytogenetics, Sullivan Nicolaides Pathology, Brisbane, Australia \\ ${ }^{2}$ Wesley Medical Centre, Brisbane, Australia}

Trisomy 9 accounts for $2.7 \%$ of all trisomic cases, and is a rare finding at prenatal diagnosis. The expectation from prenatal cytogenetic testing is for a relatively more common finding of mosaicism, with a resultant amelioration of effects, or of confined placental mosaicism with a possible associated IUGR, as most non-mosaic pregnancies result in early first trimester spontaneous abortion. There are, however, cases in the literature of apparently non-mosaic probands surviving to early infancy, and a characteristic pattern of dysmorphism and congenital abnormality seen at ultrasound. We present a case detected at CVS, with amniocentesis follow-up, and a brief literature review.

\section{CYSTIC FIBROSIS MUTATIONS IN TWO AZOOSPERMIC MALE PATIENTS}

P. Field, K. Watson, W. De Ambrosis, N. Martin

Queensland Fertility Group, Brisbane, Australia

Infertility affects approximately $15 \%$ of couples trying to conceive, with about half of these due to male infertility. Male infertility is most often due to reduced, abnormal or zero sperm production, mostly with unknown cause, although some patients have a specific structural abnormality such as absence of the vas deferens. The vas deferens connects the testicle to the outside world and allows free passage of sperm from the testicle and out of the urethra. Male patients with absence of the vas deferens present with a zero sperm count, however normal sperm are often present in the testicle and can be obtained for ICSI using MESE/TESE. Male patients presenting with azoospermia/severe oligospermia at Queensland Fertility Group are encouraged to be screened for cystic fibrosis (CF) mutations not only for the risk of carrying a CFTR mutation but also due to the association with some CF mutations and infertility. The mutation c.350G $>$ A $(\mathrm{R} 117 \mathrm{H})$ in particular has been associated with infertile male patients and is often not screened for in the general population. We present two male patients with zero sperm detected in routine sperm counts whom both have absence of the vas deferens and are both compound heterozygous c.1521_1523delCTT (F508del) / c.350G $>$ A $(\mathrm{R} 117 \mathrm{H})$. Both patients presented without any clinical symptoms or family history of CF and are both otherwise healthy.

\section{RESULTS OF A FEASIBILITY STUDY OF NEWBORN SCREENING FOR THE FRAGILE $X$ SYNDROME}

M. Field', L. Christie', J. Boyle', C. Rogers', J. Hansen', T.Wotton ²,V.Wiley ${ }^{3}$, B. Bennetts2

${ }^{1}$ Hunter Genetics, GOLD Service, Waratah, Australia

${ }^{2}$ Western Sydney Genetics Program, Molecular Genetics, Sydney, Australia

${ }^{3}$ Western Sydney Genetics Program, NSW Newborn Screening Program, Sydney, Australia

Purpose: To evaluate feasibility and maternal acceptance of newborn screening for the fragile X syndrome (FXS). Methods: 2123 women delivering at John Hunter Hospital were approached by a genetic counselor in the post natal period to participate in a FXS newborn screening trial. Screening was performed on the routine newborn screening card from male and female infants using a chimeric PCR method and repeated using a standard diagnostic PCR test. Alleles were approximately sized by non-denaturing capillary electrophoresis and those alleles suspected to have greater than 40 repeats were formally sized. Infants with allele sizes greater than 58 were recalled and results confirmed on postnatal samples. Attitudes of participating mothers to newborn screening for FXS were assessed by questionnaire. Results: 2000 mothers participated with an uptake of $94 \%$. There was complete concordance of the two testing methods. Six infants ( 5 female and 1 male) had an allele greater than 58 repeats. In four of these cases there was maternal transmission, with evidence of expansion during meiosis in one family. No affected infants were identified. 1711 (85\%) participants completed the questionnaire and $93 \%$ considered early diagnosis as beneficial to help prepare for a child with special needs and $63 \%$ would use the information when planning more children. Some participants $(9 \%)$ were anxious about the possible test results and 3\% felt their feelings towards their infant may change if found to have FXS. Conclusion: The results of this project provide the first data about the range of grey zone and premutation allele sizes seen in the general community in Australia. Our data shows newborn screening for FXS is feasible using current technology. Participation rates and results from the questionnaire indicates maternal acceptance of newborn screening for FXS despite the absence of a proven medical therapy.

\section{STAKEHOLDERS' PERSPECTIVES OF GENETICS IN PRIMARY CARE: WILL PATIENTS 'SLIP THROUGH THE CRACKS'?}

L. E. Forrest' ${ }^{\text {, B. J. McClaren }}{ }^{2,3}$, S.A. Metcalfe ${ }^{2,3}$, J. M. Hodgson ${ }^{2,3}$, J. D. Emery ${ }^{4}$

${ }^{1}$ Australian Primary Health Care Research Institute, Australian National University, Australia

${ }^{2}$ Genetics Education and Health Research, Murdoch Childrens Research Institute, Melbourne, Australia

${ }^{3}$ Department of Paediatrics, University of Melbourne, Australia

${ }^{4}$ School of Primary, Aboriginal and Rural Health Care, University of Western Australia, Australia

Background: Rapid developments in genetic technologies will change practice in all specialties of medicine, including primary care, due to the constant generation of new genetic/genomic information, genetic tests and screening procedures. However, the ability of general practitioners (GPs) to practise genetic medicine is not only dependent on adequate education but on understanding how genetic medicine can be appropriately integrated in this healthcare setting. There is concern that consumers' genetic needs will not be met by their primary care provider. The aim is to examine how genetics is currently managed in primary care from the perspective of primary care providers, genetic health professionals and consumers, and whether changes to practice will be required in the future. Methods: A qualitative approach was taken with stakeholders including primary care representatives, genetic health professionals and health care consumers. Data were collected by semi-structured interviews addressing current practice of genetics in primary care; approaches to referrals and management; views on changes to practice and suggestions for ideal and acceptable practice; models which might deliver genetics in primary care. Thematic analysis was conducted on the transcribed interviews to identify key concepts and ideas. Results and Discussion: Fourteen interviews have been conducted to date with GPs, practice nurses, genetic counselors, professional organization representatives and consumers, with more planned during this year. Preliminary findings suggest that GPs willingly engage genetic health professionals for genetic support and advice as necessary. The generalist nature of general practice, a heavy workload, and time constraints were recognized by all participants as barriers to routine integration of genetics into primary care. Concern exists that patients requiring genetics care may 'slip through the cracks', especially with regard to the urgency of testing 
in pregnancy. Nevertheless, responses were mixed regarding whether practice will need to change to successfully integrate genetics into primary care and various models of practice were discussed.

\section{FAMILY MEMBERS AS INTERPRETERS: BLURRING THE LINES BETWEEN INTERPRETER, PATIENT ADVOCATE AND PATIENT}

\section{F. Fowles, A. Hattam}

Genetic Health Queensland, Royal Brisbane and Women's Hospital, Brisbane, Australia

Genetic counselors have a responsibility to provide genetic services to all individuals in a non-discriminatory manner (ASGC Code of Ethics). When working with a patient who has limited proficiency in English, best clinical outcomes are obtained through the use of a professional interpreter. ${ }^{1}$ Professional interpreting requires impartial translating and may also involve cultural interpretation. A professional interpreter must take care not to cross the line into emotional support as this can undermine the patient's interaction with the clinician and alter the clinical outcome. ${ }^{2}$ Use of a family member as interpreter can complicate a clinical interaction, especially when a familial condition is involved. There are some advantages to using family members as trust is already established and they may be able to help the patient in navigating their clinical journey. ${ }^{3}$ However family members may have their own agendas and the lines between the interpreter's role as interpreter, patient advocate and patient can become blurred. Some of the challenges faced in such a situation are explored through a case study. A patient recently arrived from Italy attended the Marfan syndrome multidisciplinary clinic. His Australian wife acted as interpreter and their young daughter also attended. Initial concerns with this interpreting arrangement were justified when standard family history questions became awkward. Their young daughter, also affected by Marfan syndrome, was potentially exposed to higher level information about Marfan syndrome than was age-appropriate. His wife became actively involved as a co-consultant when the issue of future pregnancies was raised. Reflections on ways to better manage this scenario are presented.

${ }^{1}$ Health Service Research 2007; 42:727-754.

2 Patient Education and Counseling 2010; 81:192-197.

${ }^{3}$ Interpreting 2005; 7:77-95.

\section{FAMILY FOLLOW-UP FOR PATIENTS WITH PREMATURE CARDIOVASCULAR DISEASE AT ROYAL PRINCE ALFRED HOSPITAL, SYDNEY}

\author{
L. Freeman', C. Chow ${ }^{2}$, D. Sullivan ${ }^{3}$ \\ 1 Molecular and Clinical Genetics, Royal Prince Alfred Hospital, Sydney, Australia \\ 2 Cardiovascular Division, The George Institute for Global Health, Sydney, Australia \\ 3 Clinical Biochemistry, Royal Prince Alfred Hospital, Sydney, Australia
}

Family history of cardiovascular disease (CVD) is a sufficiently powerful predictor of future CVD to rank as one if its major classical risk factors. CVD is a multifactorial disease and there are common risk factors in a family due to genetics and shared environment, $\mathrm{m}$ any of which can be modified to reduce the future risk of CVD. The Australian PBS acknowledges this opportunity to prevent CVD stating first-degree relatives of patients with CVD before age 45 are eligible for lipid-lowering therapy. Estimates of the degree of risk associated with a positive family history vary, however it is likely that first-degree relatives of patients with premature myocardial infarction (MI) have double the risk of premature MI themselves. Families form an ideal target population for primary prevention of CVD. Unfortunately, evidence suggests that most opportunities to assess and treat relatives of patients with premature CVD are overlooked. At RPA Hospital we have developed a family orientated clinical service to meet this need. We have established a referral pattern to the genetics counselor for all patients 45 years and younger admitted with premature CVD. The genetics counselor documents the family history, discusses the multifactorial nature of CVD and explains the genetic component of CVD risk factors. The purpose of this consultation is to highlight the importance of inform- ing relatives of their CVD risk given the family history. We have produced relative notification packages containing information on premature CVD that patients can give to their relatives or ask the genetics counselor to send on their behalf. Relative notification is undertaken in a careful manner so as not cause undue anxiety; relatives receive an introductory letter, an information sheet and a letter to their GP inviting them to do a personal CVD risk assessment. The clinical service at RPA has been running for 8 months and we will present the obstacles and the outcomes of this new service.

\section{SUDDEN DEATH AND GENETIC IMPLICATIONS: EXPLORING THE EXPERIENCES OF FAMILIES IN VICTORIA}

L. Gallacher ${ }^{1,2}$, S. Metcalfe ${ }^{1,2}$, N. Morgan ${ }^{3}$, I. Macciocca ${ }^{4}$, J. Hodgson ${ }^{1,2}$

${ }^{1}$ Genetics Education and Health Research, Murdoch Childrens Research Institute, Melbourne, Australia

${ }^{2}$ Department of Paediatrics, The University of Melbourne, Australia

${ }^{3}$ Victorian Institute of Forensic Medicine, Melbourne, Australia

${ }^{4}$ Genetic Health Services Victoria, Melbourne, Australia

The genetic contribution to cardiac-related causes of sudden death is well recognized and, based on autopsy findings, cardiac screening of relatives may be recommended. In this situation the imperative for communication of genetic information within families is high. In Victoria, the potential familial implications of sudden death are communicated to the senior next of kin by forensic services personnel, who liaise with cardiac and genetics services to facilitate appropriate referral and follow-up. This research aimed to explore the experiences of family members, where the death of a relative raised the possibility of a genetic condition within the family. Seventeen relatives of individuals who died suddenly between the years 2004-2009 were interviewed in-depth. Interviews were audio-recorded and qualitative thematic analysis was conducted on transcripts. A number of important themes emerged. Participants described in detail how the shock and grief associated with the loss impacted on their ability to process complex information at the time. They expressed frustration with the lengthy forensic and genetic investigations, and explained the struggle to make meaning of their loss when a cause of death or specific diagnosis could not be made. While there was confusion surrounding the multiple processes and roles of different health professionals following the death, individuals were highly appreciative when appropriate care was provided. Participants lamented that the opportunity to be forewarned had not been available for the deceased, and this subsequently provided strong motivation for individuals to disseminate relevant health information within the family. These findings highlight the importance of collaboration between forensic and genetic services to provide streamlined care and facilitate communication among at-risk relatives.

\section{CANDIDATE GENES IN MIGRAINE}

\section{Gaspirini, B. Roy, L. Griffiths}

Genomics Research Centre, Griffith Health Institute, Griffith University, Australia

Migraine is a common debilitating primary headache disorder with significant mental, physical and social health implications. It has been documented by the World Health Organization as ranking among the top 20 leading causes of disability (Colson et al., 2006). Migraine is a complex genetic disease that affects about $12 \%$ of the Australian Caucasian population (Russell \& Olesen, 1995). Migraine is a complex neurovascular disorder that is characterized by recurrent attacks of headache, which differ in intensity, frequency and duration. The Genomics Research Centre is currently undertaking studies aimed at identifying the genes involved in the disorder. The primary aim of this research is directed towards identifying genes that play a role in migraine etiology. Migraine candidate genes are those with a known genomic location that produce biochemical and/or physiological products which could play a role in pathogenesis or pathophysiology of migraine. This study will investigate common polymorphisms within the glutamate receptor gene family specifically gria 2 and gria4. These genes are involved in binding glu- 
tamate an excitatory neurotransmitter and have been proposed as candidate genes. The role of glutamate in the pathophysiology of migraine is implicated by data from animal and human studies (Vikelis et al., 2007). Glutamate receptor antagonists have been recently proposed as a major target addressed for the treatment of migraine. RNA editing genes within the ADAR family will also be looked at due to their effect on glutamate receptor transcripts. Genotyping of four SNPs within the ADARB2 gene that have been found associated in the Norfolk Island population (Cox, 2010) will be looked at in the typical migraine population. In order to generate data for each of these SNPs, genotyping techniques including Polymerase Chain Reaction and High Resolution Melt will be utilized.

\section{SOLAR KERATOSIS (SK) SUSCEPTIBILITY: VALIDATION OF A GENOME-WIDE ASSOCIATION STUDY (GWAS)}

\section{R. Grealy', M. Kuwahata', M. Green',A. Green², L. R. Griffiths' \\ ${ }^{1}$ Genomics Research Centre, Griffith University, Gold Coast, Australia \\ ${ }^{2}$ Epidemiology and Population Health Unit, Queensland Institute of Medical Research, Brisbane, Australia}

Introduction: Solar keratosis (SK) is a skin lesion thought to be a precursor of squamous cell carcinoma (SCC), the second most common skin cancer. We wished to investigate which genes predispose patients to SK, potentially leading to SCC development. We therefore conducted a genome-wide association study (GWAS) on SK cases and controls and have individually genotyped SNPs in two genes of interest to validate GWAS findings. Methods: GWAS was performed by hybridizing SK case and control pools to Affymetrix 250K StyI SNP chips. Pools were constructed using 100 case and 100 control DNA samples were obtained from a cohort from Nambour, Queensland (collected previously). SNPs located in the DNA repair gene XRCC2 (rs3218536) and the signaling-pathway gene IGF1 (rs17727841) were selected for GWAS validation. The $\chi^{2}$ test was used to compare case and control minor allele frequencies with GWAS relative allele scores (RAS) and to compare case and control frequencies with each other. Results: For XRCC2, allele frequencies for cases were $0.95(\mathrm{G})$ and 0.05 (A), and were $0.92(\mathrm{G})$ and 0.08 (A) for controls. These allele frequencies followed the GWAS trend but were statistically different from GWAS frequencies $(p=.04)$. For IGF1, allele frequencies for cases were 0.74 $(\mathrm{C})$ and $0.26(\mathrm{G})$, and were $0.82(\mathrm{C})$ and $0.18(\mathrm{G})$ for controls. These frequencies were not statistically different from GWAS frequencies $(p=$ .07). $\chi^{2}$ analysis found no association between cases and controls for either gene (XRCC2 $p=.16$; IGF1 $p=.15$ ). Conclusions: Allele frequencies for XRCC2 and IGF1 follow the trend observed in the GWAS results, though frequency results for XRCC2 were statistically different. Additionally, case-control comparisons showed no associations, which may be due to small sample size. We intend to genotype these SNPs in a further 1097 individuals from the Nambour cohort and perform a genetic analysis incorporating known environmental SK risk factors.

\section{COMPARATIVE ANALYSIS OF CHRONIC LYMPHOCYTIC LEUKEMIA DETECTION USING FISH PANEL AND GTG KARYOTYPE.}

\section{B. Green, K. Fagan}

Cytogenetics, Pathology North(Hunter), New Lambton Heights, Australia

Chronic lymphocytic leukemia (CLL) is a disorder of the mature lymphocytes that has a heterogeneous prognosis. The median survival has increased from $\sim 5$ years in the 1970 s to $\sim 10$ years today. This is largely due to earlier diagnosis. Cytogenetics has proven to be valuable in determining the prognosis of a patient and the clinical features of the disease. Historically, karyotype analysis of bone marrow samples from CLL patients has proven to be difficult due to a low mitotic index of these mature cells and a poor response to the common mitogens. For this reason FISH has become a preferred method of detecting abnormalities in this disorder. The Cytogenetics Unit at Pathology North (Hunter) has studied CLL bone marrow samples using FISH techniques since 2003. Here we describe the evolution of the optimum FISH probes for detecting cytogenetic abnormalities in
CLL in our laboratory and, in a comparative study of FISH and GTGbanding techniques, the positive effect this has had on the abnormality detection rate and, subsequently, the classification of the patient.

BISATELLITED MARKER CHROMOSOMES IN AN INFERTILE POPULATION

N. Martin, S. Grehan

Cytogenetics, Queensland Fertility Group, Brisbane, Australia

Small supernumerary marker chromosomes are found in humans with a frequency of approximately $0.04 \%$ of newborns. The frequency is higher in prenatal specimens, mentally retarded patients and in patients with subfertility. Marker chromosomes may occur in every cell karyotyped or may occur in mosaic form. Their structure may be bisatellited with one or two centromeres, isochromosomes or have a ring structure. Supernumerary chromosomes are not easy to correlate with clinical outcome. It is generally felt that if they are inherited from a phenotypically normal parent there is no discernable risk of fetal abnormality. We undertook a retrospective survey of 7 years of infertility screening yielding approximately $0.07 \%$ of all patients presenting with a bisatellited marker chromosome, confirmed by $\mathrm{C}$ and silver banding. The sex distribution was skewed with 1 female:2males. The origin of the markers as determined by FISH using the Vysis Prader-Willi cocktail and the Poseidon 14/22 and $13 / 21$ centromere cocktail. The results showed $70 \%$ of markers to be derived from chromosome 15 and 30\% from chromosomes 14/22. This is higher than the reported figures of $50 \%$ being derived from chromosome 15 - however, this study was restricted the marker chromosomes that were bisatellited. Of those markers derived from chromosome $15 ; 25 \%$ showed signal for D15Z1 on both chromosomes 14 and 15. This latter finding is somewhat higher than the $10 \%$ reported in the literature. Sperm FISH analysis on several of the patients has shown that the marker chromosome was associated with disruption to normal chromosome segregation at meiosis and would account for some cases of a marker chromosome being associated with male infertility. This information is of use in genetic counseling for patients considering various assisted reproduction options.

\section{ADULT PRESENTATION OF GLUTARIC ACIDURIA TYPE I}

C. Gurnsey', M. Badve' , K. Beadle', C.Warnholtz', S. Bhuta ${ }^{3}$, J. McGill ${ }^{4}$

${ }^{1}$ Chemical Pathology, Central Laboratory, Pathology Queensland, Brisbane, Australia

${ }^{2}$ Department of Neurology, Gold Coast Hospital, Southport, Australia

${ }^{3}$ Department of Neuroradiology, Gold Coast Hospital, Southport, Australia

${ }^{4}$ Department of Metabolic Medicine, Royal Children's Hospital, Brisbane, Australia

Glutaric aciduria type I (GA1) is an autosomal recessive disorder of lysine and tryptophan metabolism caused by a deficiency of glutarylCoA dehydrogenase. Before the advent of extended newborn screening, patients typically presented with neurological symptoms between the ages of 6 to 18 months, the onset of which was frequently associated with intercurrent viral illness. We present a case in which the diagnosis of GA1 was not made until the fourth decade of life. Our patient, a mother of three, presented at the age of 30 years with severe migraine. A brain MRI revealed white matter changes and mild cerebral atrophy warranting further investigations. Urine organic acids were requested and showed a very large elevation of glutarate, moderate elevation of 3-hydroxyglutarate and small elevations of 2-ketoglutarate and lactate, consistent with GA1. This was further substantiated by her plasma acylcarnitine profile which showed an elevated level of glutarylcarnitine with a low free carnitine level. The patient was prescribed L-carnitine and reports that she is able to think more clearly and has had an improvement in her mood since commencing this treatment. Macrocephaly was evident on examination of the patient's head circumference, which was on the 98th centile. A review of her medical history revealed a previous episode of encephalopathy at the age of 15 years that was attributed to meningitis. Her academic performance at school was below average and she subsequently left the education system after Year 10. She has also suffered from bipolar disorder and depression. At this stage it is unclear 
whether the mental health issues are related to her metabolic disorder. Her three pregnancies were managed successfully without apparent illness. This is an unusual case of GA1 in that the patient presented at a late age with symptoms that appear to have responded to therapy.

\section{DIFFICULTIES IN THE DIETETIC MANAGEMENT OF PATIENTS WITH EARLY CHILDHOOD ONSET — MULTIPLE ACYL CO-A DEHYDROGENASE DEFICIENCY (MADD)}

K. Bhattacharya ${ }^{1,2}$, B. Hanna', S. Thompson', B. Dennison', C. Ellaway ',2, J. Christodoulou ${ }^{1,2}$, K. Carpenter ${ }^{1,2}$

1 Western Sydney Genetics Program, Children's Hospital at Westmead, Westmead, Australia

2 Department of Paediatrics \& Child Health, University of Sydney, Sydney, Australia

Background: MADD is caused by deficiency of the electron transfer flavoprotein ETF or ETFDH. A broad phenotypic spectrum due to impaired function of multiple dehydrogenases ensues. Dietary recommendations are to avoid prolonged fasting and maintain a fat and protein restricted diet, but our group describes some of the difficulties encountered. Patients: Four patients identified by the New South Wales newborn screening program with riboflavin non-responsive MADD were prospectively treated with a low fat $(<25 \%$ total energy), low protein diet. Three had symptoms of feeding difficulty, hypotonia and hypoglycemia in the neonatal period while the fourth patient developed hypotonia in the first 6 months of life. Discussion: All take 3-hydroxybutyrate and l-carnitine. The neonatal-onset patients were sensitive to dietary alteration, having frequent acute decompensations with one dying aged 13 months and the other at 9 months of age. These two had labile potassium control when unwell and had isolated low plasma histidine and intermittent grossly increased isovalerylcarnitine. The isovaleryl-carnitine became particularly elevated $(z$ score $>250)$ in both of these cases on the day of their cardiac arrests. One also had significant decrease in plasma glutamine when an essential amino acid supplement was used, improving with complete protein supplementation. The third neonatal onset patient was treated with MCT-rich formula after a severe decompensation aged 5 months, being more stable afterwards, contrary to other reports. The fourth child became profoundly hypotonic aged 6 months and improved upon introducing nocturnal uncooked cornstarch and increasing the 3-hydoxybutyrate dose. Conclusion: MADD has a broad spectrum of clinical and biochemical manifestations. The ideal dietary composition is not known and may indeed differ between patients. Acute decompensation alters flux through the pathways involved meaning that treatment may also need to change. Collaborative work and data analysis between centers may help define ideal treatments better.

\section{THIS IS M.A.D.D: A DIFFERENTIAL DIAGNOSIS FOR PRENATALLY IDENTIFIED BILATERAL MULTICYSTIC KIDNEYS}

B. Hanna', S. Rajagopalan',A.Adno',V.Wiley'

${ }^{1}$ SSWAHS, Liverpool Hospital, Sydney, Australia

${ }^{2}$ Department of Pediatrics, University of Sydney, Australia and Newborn Screening, Sydney, Australia

Multiple Acyl-CoA dehydrogenase deficency (MADD) or glutaric acduria type II (GA II) is an autosomal recessive condition affecting fatty acid,amino acid and choline metabolism, due to mutations in one of three known genes namely, ETFA, ETFB and ETFDH. There are three common clinical presentations depending on severity. They consist of Type 1, a neonatal-onset form with congenital anomalies; Type II, a neonatal onset form without congenital anomalies; and Type III, a late-onset form. MADD presents with an abnormal acylcarnitine profile detected by tandem mass spectrometry used in the newborn screening program, and a positive screen result prompts further investigations to confirm the diagnosis. We report the detection of MADD following newborn screening in a neonate with large bilateral multicystic kidneys who died shortly after birth of renal failure. The multicystic kidneys were first detected at 18 -week antenatal ultrasound,and a central cystic lesion in the brain with borderline ventriculomegaly was subsequently detected at 34 weeks gestation. The child was born to a consaguineous Lebanese couple who had a previous fetal death in utero at 28 weeks gestation with anhydramnios and bilateral mulitcystic kidneys. On both occasions, the couple declined autopsy. The differential for bilateral polycystic kidneys is extensive and prognosis depends on residual renal function and associated congenital malformations. The provisional diagnosis was autosomal recessive polycystic kidney disease, until results of newborn screen returned an abnormal result. This case outlines how the correct diagnosis was achieved following newborn screening, and the extensive differential diagnoses of antenatally detected polycystic kidneys in the offspring of a consanguineous union. The recognition of neonatal MADD would then enable genetic testing of the known MADD genes if the family desire prenatal or pre-implantation genetic diagnosis in a future pregnancy.

UNDERSTANDING THE PSYCHOSOCIAL IMPACT OF BEING DIAGNOSED, AND LIVING WITH, KLINEFELTER SYNDROME

A. S. Herlihy ${ }^{1,2,3,4}$, J. L. Halliday ${ }^{1,5}$, R. I. McLachlan ${ }^{2,3,4}$, L. Gillam ${ }^{5,6}$

${ }^{1}$ Public Health Genetics, Murdoch Childrens Research Institute, Melbourne, Australia

${ }^{2}$ Obstetrics \& Gynaecology, Monash University, Australia

${ }^{3}$ Clinical Andrology, Prince Henry's Institute of Medical Research, Melbourne, Australia

${ }^{4}$ Andrology Australia, Melbourne, Australia

${ }^{5}$ The University of Melbourne, Australia

${ }^{6}$ Children's Bioethics Centre, Melbourne, Australia

Klinefelter syndrome (KS) is a genetic condition affecting males (47XXY), resulting in a spectrum of clinical features ranging from infertility, androgen deficiency, and gynecomastia to social, behavioral and learning difficulties. The prevalence has been estimated at 1:650, yet up to $70 \%$ remain undiagnosed. While the medical aspects of KS have been explored, almost no evidence exists regarding the personal impact of KS. This study aimed to explore the psychosocial impact of being diagnosed and living with KS, and the attitudes of men with KS towards optimal diagnosis age, acceptability of screening, and effectiveness of therapeutic interventions. Thirty-seven men from a larger questionnaire study were interviewed and sessions were audio-taped, transcribed and analyzed for content, interactions and themes. Analysis revealed a wide range of reactions to the diagnosis of KS and a variable personal impact of living with the condition. The majority of participants were diagnosed in adulthood and discussed the devastation of not only being diagnosed as infertile, but as also having 'a genetic condition'. This often led to an attempt to try and make sense of 'How much is me and how much is KS?' The diagnosis prompted many men to contemplate their sexual and gender identity. Most felt early diagnosis was best and supported population screening for KS, but found it difficult to reflect how their own lives may have been different if they had been diagnosed earlier. These reflective processes often occurred in the absence of access to resources or support, highlighting gaps requiring further attention.

CASCADE SCREENING OF FAMILY MEMBERS FOLLOWING A HIGH SCHOOL SCREENING PROGRAM FOR HEREDITARY HEMOCHROMATOSIS

C. L. Hickerton', E.Varley', M.Wolthuizen', K. Trembath',V. Collins',

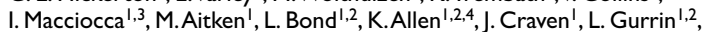

P. Lockhart', G.Wilson', S. Stephenson', M. Delatycki ${ }^{1,2,5}$, S. Metcalfe ${ }^{1,2}$

${ }^{1}$ Murdoch Childrens Research Institute, Melbourne, Australia

${ }^{2}$ The University of Melbourne, Australia

${ }^{3}$ Genetic Health Services Victoria, Melbourne, Australia

${ }^{4}$ The Royal Childrens Hospital, Australia

${ }^{5}$ Austin Health, Melbourne, Australia

Population screening can give family members of a tested individual the opportunity to access cascade screening to find out about a potentially unknown genetic predisposition in their family. We present the findings of cascade testing as a result of the ironXS study. Briefly, 5757 students were tested, identifying 28 HFE C282Y homozygotes who all attended genetic counseling sessions together 
with their parents, and $586 \mathrm{C} 282 \mathrm{Y}$ heterozygotes, who received their result by mail. 142 interviews were conducted with students found to be homozygous $(n=25)$, and a sample of heterozygous $(n=17)$ and 'wild type' students $(n=26)$ and their parents $(n=74)$. Information about cascade screening was gathered during interviews and also from genetic counseling sessions with families. In 117 interviews communication of genetic information with relatives and cascade testing was discussed. Students generally reported not playing a role in telling relatives $(n=37 / 55)$. Half of the parents discussed giving relatives information about cascade testing $(n=31 / 62)$. Reports of family members being tested were made by 10 parents of homozygotes, 4 heterozygotes and no parents of 'wild types'. Qualitative analysis revealed communication of the information appeared to be dependent on how proactive the parents were and whether they were in contact with their extended family. Participants who reported cascade testing within their families expressed an appreciation of the ability to pass on this knowledge. In some cases this helped families with undiagnosed illness find the missing piece. This shows evidence that population screening and subsequent cascade testing can give people potentially helpful knowledge that they would not have normally accessed.

\section{THE IMPACT OF ARRAY CGH TESTING ON A DIAGNOSTIC CYTOGENETIC LABORATORY.}

T. Hocking, R. Buchheim, R. Coates, C. Derwas, P. Dubois, L. Gowans, H. Kais, A. Marks, J. Samuel, S. Smith, L. Teicher

Cytogenetics Unit, Genetic Medicine, SA Pathology, Adelaide, Australia

Diagnostic testing using CGH array was introduced to this laboratory in July 2010 for all patient referrals for developmental delay, intellectual disability, autism or at least two congenital abnormalities. In the first 6 months following implementation there have been $922(52 \%)$ specimens for cytogenetic analysis and $844(48 \%)$ array tests performed. The abnormality rates for the time intervals JulyDec 2009 and July-Dec 2010 are both approximately 5\% but the indications for the 6 months Jan-June 2011 are that the number of abnormalities has decreased significantly in the chromosome analysis category. Comparisons using statistics for all specimens received by June 30, 2011 will be tabled. These will include a breakdown of referral reasons and abnormalities (number and type) for each form of testing. One dilemma has been deciding which test is more appropriate for babies (aged less than 6 months). If the request form clearly indicates a possible diagnosis that lends itself to chromosome analysis (e.g., Down syndrome), that is the test performed. If the clinical details meet the 2 or more congenital abnormality criteria, the sample is sent for array. If the details are ambiguous, a phone call to the doctor usually resolves the issue. One possible exception is testing for $22 \mathrm{q}$ microdeletion syndromes. These specimens have an array but a FISH test is performed in the interim if an urgent result is required. Family follow up with FISH has added an additional 154 specimens in the 6 months following implementation of array testing. Another source of additional specimens in this period is patients that have been reported to have no abnormality with previous cytogenetic testing. The impact on staffing, resources and laboratory management has been dramatic with referrals for array $\mathrm{CGH}$ and bloods being higher than original estimates. The implementation of array $\mathrm{CGH}$ will continue to challenge the daily management of the diagnostic cytogenetics laboratory as additional referral categories are triaged to this technology for first tier testing.

\section{REFLECTIVE PRACTICE: USING SIMULATED PATIENTS IN} GENETIC COUNSELING TRAINING PROGRAMS

\author{
J. Hodgson ${ }^{1,2}$, M. Sahhar ${ }^{2,3}$, J. Barrett ${ }^{2}$ \\ ${ }^{I}$ Genetics Education and Health Research, Murdoch Childrens Research Institute, \\ Melbourne, Australia \\ ${ }^{2}$ Department of Paediatrics, University of Melbourne, Australia \\ ${ }^{3}$ Genetic Health Services Victoria, Melbourne, Australia
}

Reflective practice is an extremely important part of genetic counseling training and professional development. The ability to reflect upon interactions with clients is a vital skill for all genetic counselors. As part of the 2 year Master of Genetic Counseling program at the University of Melbourne/MCRI we developed a simulated patient (SP) programme to enhance counseling skills and encourage self reflection. Based on the concept of experiential learning, the SP program utilizes trained actor-tutors who improvise a scenario with a student and provide live feedback. Students are given a DVD of the session to allow further reflection, both alone and with a supervisor. A written account of the student's reflection is subsequently used for assessment. Since 2009 we have recorded more than 40 SP sessions and feedback from our students has been overwhelmingly positive. This presentation will outline the development and ongoing refinement of the SP program in Melbourne and, using visual examples of recorded scenarios, demonstrate how this learning experience can enhance counseling skills training. In addition we will present a useful guide for others who wish to use SP programs in genetic counseling training.

EFFECT OF TETRAHYDROBIOPTERIN ON PHE/TYR RATIOS AND VARIATION IN PHE LEVELS IN TETRAHYDROBIOPTERIN RESPONSIVE PKU PATIENTS

M. Humphrey ${ }^{1,2}$, J. Nation 1,2, I. Francis ${ }^{3}$,A. Boneh 1,2,3,4

1 Metabolic Service, Genetic Health Services Victoria, Melbourne, Australia 2 Department of Nutrition \& Food Services, Royal Childrens Hospital, Melbourne, Australia

3 Newborn Screening Laboratory, Murdoch Childrens Research Institute, Melbourne, Australia

4 Department of Paediatrics, University of Melbourne, Australia

Background: While a reduction in blood phenylalanine (Phe) levels is essential in patients with PKU, a decrease in Phe/Tyrosine (Tyr) ratio and in fluctuations in blood Phe levels over time have been recently associated with improved neuropsychological outcome. The aim of this study was to identify if Tetrahydrobiopterin (BH4) offers additional benefit based on the assumption that these 2 factors are beneficial. Method: Since 2002, 9 patients identified through NBS as $\mathrm{BH} 4$ responsive (BH4) and 25 non-responsive patients (non-BH4) produced a total of 1384 and 4415 samples, respectively, for analysis. Statistical analysis was performed to compare mean and median Phe levels, Tyr levels and Phe/Tyr ratios in BH4 and non BH4 responsive patients. Results: Variations in blood Phe levels were

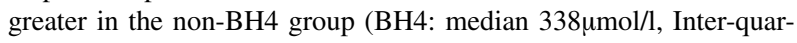
tile range (IQR) 192, mean: $358 \mu \mathrm{mol} / \mathrm{L}$, SD 158; non-BH4: median $337 \mu \mathrm{mol} / \mathrm{l} \mathrm{IQR} \mathrm{263,} \mathrm{mean:} 370 \mu \mathrm{mol} / \mathrm{L}$ SD 204). Variations in blood Tyr levels were slightly greater in the non-BH4 group: (BH4: median $59 \mu \mathrm{mol} / \mathrm{l} \mathrm{IQR} \mathrm{38,} \mathrm{mean} 67$ SD 30; non-BH4: median 62 $\mu \mathrm{mol} / \mathrm{l} \mathrm{IQR}$ 38 , mean $70 S D 35$ ). The variation in phe/tyr ratios was greater in the non-BH4 group (Mean 5.78, SD 3.17,\%CV 54.8) vs. (mean 6.12, SD 4.4, \%CV 71.9), particularly at blood Phe levels $>600 \mu \mathrm{mol} / \mathrm{l}$. Conclusion: $\mathrm{BH} 4$ responsive patients have smaller variations in blood Phe levels and tighter Phe/ Tyr ratios than non-BH4 responsive patients, particularly at high blood Phe levels. If decreased fluctuations in Phe levels and a decreased Phe/Tyr ratio are indeed neuro-protective, then $\mathrm{BH} 4$ responsiveness is advantageous over diet alone in PKU. Neuropsychological testing in patients who have been treated with $\mathrm{BH} 4$ long term may be able to ascertain the clinical benefit of these biochemical findings.

SIBLING COMPARISON STUDY OF 7 YEARS OF ENZYME REPLACEMENT THERAPY FOR MUCOPOLYSACCHARIDOSIS TYPE VI STARTING AT 8 WEEKS AND 3.5 YEARS OF AGE.

A. C. Inwood', D. J. Coman', J. Cramb², J.J. McGill'

${ }^{1}$ Metabolic Medicine, Royal Children's Hospital, Brisbane, Australia

${ }^{2}$ Physiotherapy, Royal Children's Hospital, Brisbane, Australia

Two siblings with mucopolysaccharidosis type VI (Maroteaux-Lamy syndrome) have had 7 years treatment with enzyme replacement therapy (ERT) using galsulfase (Naglazyme) weekly at a dose of $1 \mathrm{mg} / \mathrm{kg}$ starting from 8 weeks of age and 3.5 years of age respectively. Treatment has been well tolerated by both siblings with no 
infusion associated reactions recorded. In the younger sibling, ERT has preserved joint movement, cardiac valves, liver and spleen size, height (10th centile) and facial morphology. He has mild scoliosis (23 degrees), stable mild corneal clouding but has recently developed evidence of carpal tunnel syndrome and has significant skeletal disease with a waddling gait from avascular necrosis of the hips. The older sibling initially had a marked improvement in joint and scoliosis mobility which have been maintained and has softening of her facial features and stabilized cardiac valve pathology and corneal clouding. Her height is $6 \mathrm{cms}$ below the 1st centile after correcting for scoliosis which has progressed. Rodding of her spine, which was considered necessary prior to initiating ERT, has been deferred until completion of growth. She has required surgery for severe pes cavus. This paper adds further evidence that early initiation of ERT will slow or prevent the natural pathological progression of MPS VI.

\section{A COMPARATIVE STUDY OF HIGH RESOLUTION MELT ANALYSIS AND PYROSEQUENCING FOR THE DETECTION OF KRAS MUTATIONS IN PARAFFIN-EMBEDDED TUMOR TISSUES}

V. Jones, T. Hunt, J. Harraway, S. Byrne

Molecular Pathology, Sullivan Nicolaides Pathology, Brisbane, Australia

KRAS mutation status can be used as a predictive marker to determine the response of colorectal carcinomas to epidermal growth factor receptor (EGFR) therapies. The detection of mutations in codons 12 and 13 of the KRAS gene was compared between high resolution melt (HRM) analysis and pyrosequencing. 132 samples were tested in parallel and found to be $97 \%$ concordant. 48/132 $(36.4 \%)$ patients were identified as carrying a mutation within codon 12 or 13 of the KRAS gene, with codon 12 being the most frequently affected. Mutations involving $\mathrm{G}>\mathrm{A}$ and $\mathrm{G}>\mathrm{T}$ transitions were of the highest frequency. Mutant and wild-type oligonucleotides of the codon 12 and 13 region of the KRAS gene were then used to determine sensitivity of each assay. The allele quantification method on pyrosequencing allowed accurate proportions of mutant and wildtype alleles to be determined which was useful for assay validation and quality assurance. Furthermore, pyrosequencing provided precise mutation information which may be of importance in predicting response to treatment and clinical survival status of patients. Overall analysis of KRAS mutation status by pyrosequencing was found to be a simple, robust, fast, cost-effective and sensitive method that was also shown to be suitable for use with paraffin-embedded tissues.

\section{RISK OF ENDOMETRIAL CANCER IN EPCAM DELETION- POSITIVE PATIENTS: A NEW ASSAY}

M. Kaps, V. Calabro, D. Du Sart

Molecular Genetics Laboratory, VCGS Pathology, Murdoch Childrens Research Institute, Royal Children's Hospital, Melbourne, Australia

Hereditary non-polyposis colorectal cancer (HNPCC) is a dominantly inherited cancer syndrome caused by germline mutations in the DNA mismatch-repair genes MLH1, MSH2, MSH6 and PMS2. HNPCC is characterized by a high risk of early onset colorectal cancer and several other extracolonic malignant tumors, especially endometrial cancer. People who are carriers of mutations in MLH1, MSH2 or MSH6 have a 30-80\% risk of colorectal carcinoma by the age of 70 years. Women with HNPCC have an additional $27-71 \%$ risk of endometrial cancer by this age. Deletions of the 3 ' end (exons 8 and 9) of the EPCAM gene are a novel cause of HNPCC. These EPCAM deletions result in transcriptional read-through into the neighbouring gene, causing methylation of the MSH2 promoter and ultimately silencing of the MSH2 gene. A recent study of such EPCAM deletions reported that endometrial tumors occurred only in patients with EPCAM deletions extending close ( $<2.5 \mathrm{~kb}$ upstream) to the MSH2 promoter region, suggesting that an increased risk of endometrial cancer is dependent on the location of the EPCAM deletion. ${ }^{1}$ We developed a new PCR based assay to determine whether an EPCAM deletion extends into the region upstream of the MSH2 pro- moter. We screened our EPCAM deletion-positive cohort using this new assay and were able to establish the risk of endometrial cancer for all our patients. Although more clinical data is needed to set up criteria for the clinical management of these patients, it seems reasonable to suggest that surveillance and preventative surgery for endometrial cancer could be omitted for those patients with EPCAM deletions extending a long way from the MSH2 promoter. Here we will present the method used to detect the location of the EPCAM deletion and report on the findings of our cohort.

${ }^{1}$ Lancet Oncology 2011; 12:49-55.

\section{A CASE EXAMPLE OF DOUBLE RECIPROCAL INSERTIONAL} TRANSLOCATION

A. L. Kelly', M.T. Gabbett ${ }^{1,2}$, J. M. McGaughran ${ }^{1,2}$

${ }^{1}$ Genetic Health Queensland, Royal Brisbane and Women's Hospital, Brisbane, Australia ${ }^{2}$ School of Medicine, The University of Queensland, Australia

Reciprocal insertional translocation is a rare event with a prevalence of 1 in 80,000 cases. Following is a case of double reciprocal insertional translocation (DRIT), although with an unknown incidence it is presumed to be an exceedingly rare event. In our case offspring inherited opposite unbalanced paternal segregants. Paternal and offspring karyotypes will be further discussed. In the paternal karyotype there were two copies of JAG1 locus detected: one at the normal location of 20p12 and one inserted at the long arm of chromosome 5 at $5 \mathrm{q} 12.1$. Sibling A presented with Alagille syndrome was found to have an interstitial deletion of $5 \mathrm{q} 12.1 \mathrm{q} 13.2$ and an interstitial duplication of $20 \mathrm{p} 12.3 \mathrm{p} 12.1$. Sibling B presented with dysmorphism and neuromigrational disorder and was found to have interstitial duplication of 20p12.3p12.1 and interstitial deletion of $5 \mathrm{q} 12.1 \mathrm{q} 13.2$ detected on microarray. We present this case as a rare example of a double reciprocal insertional translocation.

\section{A POLYMORPHIC MIR-155 BINDING SITE IN AGTR1 IS ASSOCIATED WITH CARDIAC HYPERTROPHY IN PEOPLE WITH FRIEDREICH ATAXIA \\ M. Kelly ${ }^{1,2}$, R. D. Bagnall', R. E. Peverill ${ }^{3}$, L. Donelan ${ }^{3}$, L. Corben ${ }^{4}$, M. B. Delatycki ${ }^{4,5}$, C. Semsarian ${ }^{1,2,6}$ \\ ${ }^{1}$ Agnes Ginges Centre for Molecular Cardiology, Centenary Institute, Sydney, Australia \\ ${ }^{2}$ Sydney Medical School, University of Sydney, Australia \\ ${ }^{3}$ Monash Cardiovascular Research Centre, MonashHEART and Monash University Department of Medicine (MMC), Australia \\ ${ }^{4}$ Murdoch Childrens Research Institute, Melbourne, Australia \\ ${ }^{5}$ Clinical Genetics, Austin Health, Melbourne, Australia \\ ${ }^{6}$ Department of Cardiology, Royal Prince Alfred Hospital, Sydney, Australia}

Background: Friedreich ataxia (FRDA) is a neurodegenerative condition with a heterogeneous cardiac phenotype caused primarily by an expanded GAA trinucleotide repeat in the FXN gene. The number of GAA repeats on the smaller FXN allele (GAA1) only accounts for a portion of the observed variability in cardiac phenotype. Genetic modifying factors, such as single nucleotide polymorphisms (SNPs) in the Renin-Angiotensin-Aldosterone system (RAAS), may contribute to phenotype variability. This study investigated genetic variability in the angiotensin-II type-1 receptor (AGTR1), angiotensin-converting enzyme (ACE), and ACE2 genes as cardiac phenotype modifying factors in FRDA patients. Methods: Comprehensive review of the AGTR1, ACE and ACE2 genes identified 12 haplotype tagging SNPs. Correlation of these SNPs with left-ventricular septal wall thickness (SWT), left ventricular mass (LVM) and left ventricular internal diameter in diastole (LVIDd) was examined in a large Australian FRDA cohort $(n=79)$ with adjustments performed for GAA1, GAA2, body surface area, sex, diastolic blood pressure and duration of symptoms. Results: The AGTR1 polymorphism rs5186 was more common in FRDA patients than in a control population (minor allele frequency: $0.35 \pm 0.04$ vs. $0.22 \pm 0.02 ; p=.002$ ). Using a recessive model of inheritance, the $\mathrm{C}$ allele of rs5186 showed a significant increase in SWT $(\mathrm{AA}+\mathrm{AC}=10.7 \pm 2.1 \mathrm{~mm}, \mathrm{CC}=11.7 \pm 2.8 \mathrm{~mm} ; p=.005)$ and $\mathrm{LVM}$ (AA+AC $=172 \pm 54 \mathrm{~g}, \mathrm{CC}=208 \pm 48 \mathrm{~g} ; p=002)$. This functional polymor- 
phism increases expression of AGTR1 by altering the binding site for miR-155, a regulatory microRNA. No associations with LV structure were observed for the remaining RAAS polymorphisms. Conclusions: The AGTR1 polymorphism rs5186 had an increased frequency in a large FRDA population and was associated with increased SWT and LVM. This study supports the role of RAAS polymorphisms as modifiers of cardiac phenotype in FRDA patients.

\section{RCPA/HGSA MOLECULAR GENETICS QUALITY ASSURANCE PROGRAM - MOVING FORWARD.}

C. Kennedy', D. DuSart ${ }^{2}$, B. Bennetts ${ }^{3}$, J. Christodoulou ${ }^{3}$

1 RCPA/HGSA Molecular Genetics QAP, The Royal College of Pathologists of Australasia, Sydney, Australia

2 Molecular Genetics Laboratory, Victorian Clinical Genetics Service, Murdoch Institute, Royal Childrens Hospital, Melbourne, Australia

3 Western Sydney Genetics Program, The Children's Hospital at Westmead, Sydney, Australia

Molecular genetic testing has become an important component of diagnostic testing for many inherited and somatic disorders. The rapidity of technical advances and growth in the discovery and complexity of molecular disease pose significant challenges for testing laboratories to ensure integrity, validity and utility of their test procedures. It is also important that diagnostic laboratories keep abreast of the latest findings and opinions on the clinical interpretation of test results. The HGSA Molecular QAP had been in operation from 2000 to 2009, providing external QAP for laboratories involved with molecular genetic testing for inherited conditions. However, to manage the ever increasing challenges of providing a QAP that can embrace the burgeoning technology and expand into new services and achieve accreditation of the program, a Joint Molecular QAP offered by both the HGSA and RCPA QAP was considered. In 2010, the Royal College of Pathologists of Australasia and the Human Genetics Society of Australasia joined expertise to coordinate an expanded human molecular genetics quality assurance program to facilitate the essential requirement for continual laboratory improvement. In this poster, an outline of the strategic plan for the expansion of the RCPA/HGSA Molecular Genetics Quality Assurance Programme will be reported, along with a review of the overall performance of Australasian laboratories in comparison to our international counterparts. In addition, proposed future directions of the quality assurance program will be outlined.

\section{PEER LIVE SUPERVISION IN THE GENETIC COUNSELING} SETTING

M. Kentwell', L. Hodgkin',A. Sexton',Y. Bylstra', M. Bogwitz', K. Mann', J.Taylor', M. Sahhar' ${ }^{2}$, F. Pearce', G. J. Lindeman', I. Winship'

${ }^{1}$ Genetic Medicine and Familial Cancer, Royal Melbourne Hospital, Melbourne, Australia ${ }^{2}$ Victorian Clincial Genetics Services, Genetic Health, Melbourne, Australia

At the Royal Melbourne Hospital, the Genetic Counselors identified the desire to learn from each other, and engage in new forms of learning. Subsequently, a pilot project focusing on reflection, accountability, and experiential learning was developed. Live supervision with peers was identified as the model which fitted the learning objectives of the team. The aims of the project were to:

1. develop a live peer supervision model relevant to genetic counseling practice applicable to both Board Eligible and Certified Genetic Counselors

2. develop guided post observation questions based on reflective practice

3. identify key learning points from the live peer supervision sessions.

Seven Associate Genetic Counselors/Genetic Counselors participated in the project. The first phase involved collaborative brainstorming sessions to define the purpose of the project, gain knowledge and skills relevant to supervision, set the supervision contract, and develop post-observation questions. After each live supervision session, the discussions of the observer and observee were audiorecorded. The second phase of the project involved analysing the recorded discussions and identifying key areas of learning relevant clinical practice. The observer and observee's selfreported areas of learning were also identified. This presentation will describe the process and outcomes from these two phases of the project. This pilot project provided the genetic counselors with an opportunity to enhance clinical and supervision skills, harness the skills brought by each genetic counselor, learn from one another's qualities, increase awareness of one's own style in clinical practice, and engage in the reflective process with immediacy. There were a number of learning areas unique to this model of supervision, including access to experiential and visual learning. Live-peer supervision may be a useful model to consider for both Associate and Certified Genetic Counselors to engage in their continuing development and growth in clinical and supervision skills, ultimately improving patient care.

\section{CHIMERISM IN TWO SETS OF MONOCHORIONIC DIZYGOTIC TWINS FROM SPONTANEOUS PREGNANCIES. IS CHIMERISM IN TWINS RARE OR SIMPLY UNRECOGNIZED?}

A. Kidd',A. Raisis', J.Watt', M. Robertson', B. Dixon ${ }^{2}$, N.Austin ${ }^{2}$, R. Reid ${ }^{2}$, J. Gearry ${ }^{3}$, K. Badami ${ }^{4}$

${ }^{1}$ Canterbury Health Laboratory, Canterbury District Health Board, Christchurch, New Zealand

${ }^{2}$ Paediatric Department, Canterbury District Health Board, Christchurch, New Zealand ${ }^{3}$ Pathology Department, Canterbury District Health Board, Christchurch, New Zealand ${ }^{4}$ Blood Transfusion Service, Christchurch, New Zealand

Monochorionic dizygotic (MC DZT) twin pregnancies are thought to be extremely rare in spontaneous pregnancies but may be seen more commonly after assisted reproductive technology. A chimera is an individual who has cells from more than one individual. Most cases are iatrogenic after cell and/or tissue transplantation. Chimerism in twins has been reported occasionally and is thought to be due to blood vessel anastamoses in MC DZT. However, molecular studies have rarely looked at more than two different tissues in humans. Chimerism can also occur through fertilization of two ova by two sperm, followed by fusion of the zygotes and development of an organism with intermingled cell lines. It has also been suggested that sometimes two zygotes could come together and then split again resulting in a twin pregnancy with extensive chimerism. Blood chimerism in the female twin of opposite sex twins can sometimes lead to hermaphroditism in humans, and this is almost always the case in the female twin of opposite sex twins in cattle. The hermaphroditism is thought to be due to the passage of male hormones into the female circulation. Much of the literature on chimerism and twinning is on animals. Chimerism in the marmoset has been shown to occur in a wide variety of tissues, including the germ cells, in many dizygotic twins. We present two sets of twins who are MC DZT from spontaneous pregnancies from one hospital. Both sets of twins were opposite sex. Chorionicity was confirmed on placental histological analysis. We present cytogenetic, molecular and blood group results on the twins which confirm that the twins are chimeras in blood cells but not in buccal cells and we present molecular results from a wide range of tissues in one of the twins supporting the view that the chimerism is confined to the blood cells in both sets of twins.

\section{PERRY SYNDROME IN A NEW ZEALAND FAMILY PRESENTING WITH CENTRAL HYPOVENTILATION, WEIGHT LOSS AND SOCIAL WITHDRAWAL}

\footnotetext{
A. M. J. Kidd', M. Hlavac ${ }^{2}$, K. Mina', A. Raizis'

${ }^{1}$ Canterbury Health Laboratory, Canterbury District Health Board, Christchurch, New Zealand

${ }^{2}$ Respiratory department, Canterbury District Health Board, Christchurch Hospital, New Zealand
}

Perry syndrome is an autosomal dominant, neurodegenerative disorder associated with central hypoventilation, mild Parkinsonism, weight loss, depression, apathy, social withdrawal and autonomic disturbances. It is caused by mutations in the DCTN1 gene. It has 
been reported in only eight families world-wide and we present the first Australasian family with this condition to our knowledge. The proband, aged 58, gave a 4-year history of daytime somnolence and nocturnal breathing disturbance. He was referred to the Sleep Clinic where he was found to have severe central sleep apnoea and type 2 respiratory failure. He has been on nocturnal BiPAP since then, with marked improvement in both respiratory function and alertness, and has returned to full-time employment. The proband has had unexplained weight loss of $6 \mathrm{~kg}$ associated with muscle wasting despite eating large quantities of food. He had a long-standing mild tremor. His wife described how he had become socially withdrawn over the last 5 years but he denied feeling depressed. He had a marked postural drop in blood pressure, episodic tachynoea at rest and a blank facial expression, but an otherwise normal neurological examination. He had a normal brain MRI. He is heterozygous for a c.182A $>\mathrm{G}$ mutation (p.Tyr61Cys) in the DCTN1 gene. This novel variant is in a highly conserved part of the gene. The proband's father died aged 66 of an undiagnosed progressive neurological disorder associated with inadequate ventilation, autonomic disturbances, expressionless face and intermittent absent upward gaze. We present the postmortem details of the father's brain and further clinical details about the father and son and review of the literature.

\section{MILD ELEVATIONS IN CITRULLINE ON NEWBORN SCREENING AND A COMMON MUTATION INFERRING BENIGN CITULLINEMIA TYPE 1}

D. P. Knoll', R. Marquis-Nicholson ${ }^{2}$, E. Glamuzina ${ }^{2,4}$, D. R. Love ${ }^{2,4}$, C.Wilson ${ }^{1,3}$

${ }^{1}$ National Testing Centre, LabPlus, Auckland City Hospital, Auckland, New Zealand

${ }^{2}$ Diagnostic Genetics, LabPlus, Auckland City Hospital, Auckland, New Zealand

${ }^{3}$ Paediatric Metabolic Service, Starship, LabPlusLabPlus, Auckland City Hospital, New Zealand

${ }^{4}$ School of Biological Sciences, University of Auckland, New Zealand

Background: Citrullinemia Type 1 is a rare autosomal recessive disorder of intermediary metabolism caused by deficiency in the urea-cycle enzyme arginosuccinate synthase (ASS). A heterogeneous group of over 87 mutations in the ASS1 gene are known to cause the classical phenotype of encephalopathy with hyperammonemia, hypercitrullinemia (greater than $1000 \mathrm{~m} \mathrm{~mol} / \mathrm{L}$ ) with resultant death or significant neurodisability. Recently we have reported three children of Pacific Island decent who had mildly elevated levels of citrulline on expandednewborn screening (E-NBS). These three patients were found to be homozygous for a mutation c.787G $>$ A (p.Val263Met) that had previously only been reported in patients with benign, asymptomatic disease. All three had been followed for over two years and have remained clinically and biochemically well. Methods and Results: Five further children have had citrulline levels greater than our cut off range (normal $<100 \mu \mathrm{mol} / \mathrm{L}$ ) measured via MS/MS during E-NBS. All children had Tetra-ARMS PCR looking for the common mutation only (c.787G $>$ A) and all were homozygous. All children have remained asymptomatic. Two did not have a second tier blood spot card requested but went straight to molecular work-up. No children with classical citrullinemia type 1 or an elevated citrulline due to another inborn error of metabolism have been found on E-NBS since screening commenced in 2006. Conclusions: The c.787G $>$ A mutation appears to be common in the Pacific Island community. Given seemingly low levels of clinical presentation with hyperammonemia in the general population and the frequency with which the mildly elevated citrulline is being found on ENBS we suggest a change to the screening protocol to proceed directly to molecular diagnosis with the potential to not report this as an abnormal result if this common mutation is found.

\section{MUTATION SCREENING OF MULTIPLE GENES IMPLICATED} IN LONG QT SYNDROME.

\section{S. Lai, C. Lan, R. Marquis-Nicholson, J. Love, E. Doherty, D. R. Love \\ Diagnostic Genetics, LabPlus, Auckland District Health Board, Auckland, New Zealand}

Mutation screening of multiple genes is a challenging task in a diagnostic environment. Our analysis of the genes implicated in Long
QT syndrome has led to the development of a rapid means of screening for point mutations and deletion/duplication events. The former currently relies on conventional Sanger sequencing, but with a more streamlined front-end of amplification and clean-up, together with improved sequence analysis and an automated bioinformatic approach to evaluating unclassified variants. We are also complementing this strategy with an exome capture and parallel sequencing approach using a GS-Junior platform. The screening for deletion and duplication events has involved the construction of a gene-focussed custom array that enables the simultaneous evaluation of approximately 80 genes. This array-based method has replaced multiplex ligation-dependent probe amplification (MLPA) as the dosage assayof-choice.

\section{A NOVEL SUSCEPTIBILITY LOCUS FOR FOCAL AND SEGMENTAL GLOMERULOSCLEROSIS MAPS TO CHROMOSOME X021-24 IN AN AUSTRALIAN PEDIGREE}

R. Lea', H. Cox', B. Maher', D. Moses', S. Quinlan', T. Esposito², F. Gianfrancesco², L. Griffiths

${ }^{1}$ Genomics Research Centre, Griffith Health Institute, Gold Coast, Australia

${ }^{2}$ Molecular Genetics and Genomics Laboratory, Institute of Genetics and Biophysics, Naples, Italy

Background: Focal segmental glomerulosclerosis (FSGS) is a renal disorder causing kidney failure and associated heart disease. The renal pathology underlying FSGS has molecular and pathological features in common with diabetic nephropathy, a leading and increasingly prevalent cause of end stage renal disease. There are currently several known genetic causes of the hereditary forms of FSGS but the full extent of disease etiology is not yet known and thus treatment is limited. Methods: We ascertained DNA and clinical phenotype information from a large Australian pedigree with a prevalence of FSGS and co-segregating heart block disorder. The pedigree - containing only affected males — suggests the disease is inherited in an X-linked recessive manner. Comorbid heart block and $\mathrm{x}$-linked inheritance have not been previously observed for familial FSGS. To identify disease susceptibility genes we performed a linkage study of the pedigree using 47 microsatellite markers spanning the entire $\mathrm{X}$ chromosome. Results: Non-parametric multipoint linkage analysis using the MERLIN program revealed a statistically significant linkage peak at Xq21.33. Specifically a maximum LOD score of $3.32(P=5 \times 10-5)$ was obtained for marker DXS1106. Haplotype sharing analysis indicates the disease gene(s) resides within a $21.2 \mathrm{cM}$ region flanked by markers DXS8077 and DXS8064. Conclusions: These results provided convincing evidence for a novel X-linked gene(s) for FSGS and co-morbid heart block segregating in this Australian pedigree. We are currently performing a complete exome screen to identify the causative gene and mutation(s). Identifying the genetic pathways that are disrupted in FSGS will be important for understanding the disease pathology and may also help explain common forms of related kidney and heart disease, ultimately leading to better treatments.

\section{MULTI-OMICS LANDSCAPE OF CLEAR CELL RENAL} CARCINOMA

J. Li,W. Lui, J.Wang

BGI Shenzhen, Shenzhen, China

Tumor is considered to be a systematic disorder of molecular network in tissue cells, while the incidence of renal cell carcinoma (RCC) has a significant and continually increasing trend. In our study, we present a comprehensive (more than $250 \mathrm{Gbp}$ ) data set from whole genome, DNA methylome and transcriptome sequencing of RCC. By comparison between RCC and corresponding para-carcinoma (PARA) tissues, we identified 43428 single nucleotide variations (SNVs), with 168 in coding region, 5448 insertions and deletions (indels, 1-10 bp) and 9095 segmental rearrangements or copy number changes (SVs/CNVs, $1 \mathrm{k}-50 \mathrm{k} \mathrm{bp}$ ). We discovered 24187 differentially methylated regions and 8416 differentially 
expressed protein-coding genes as well as non-coding RNAs between RCC and PARA. We attempted to integrate all information from these omics levels to detect the inter-correlations in-between, and the discovered patterns are associated with Focal adhesion, MAPK signaling and other pathways related to cell metabolism, proliferation and development. The methylation pattern and expression level indicates IFI16 gene, which encoding interferon, gammainducible protein 16 , not only modulates p53 function, but also plays an important role, as a hub gene, in apoptosis pathway. Our results demonstrate an initial investigation of whole omics landscape of tumor tissues and pave a new way to understanding the important disease by multi-omics sequencing approaches.

\section{THE APPLICATION OF EXOME SEQUENCING IN DISEASES}

J. Li,W. Lui,W. Jun

Beijing Genomics Institute, Shenzhen, China

Next-generation technologies have reduced the costs of highthroughput sequencing by several orders of magnitude and have allowed for the whole-genome sequencing of several human genomes. However, whole-genome sequencing of the large numbers of individuals needed for studies of population genetics or trait associations remains unaffordable. One alternate approach, taken by the 1000 Genomes Project, is low-pass sequencing (with an average of fourfold genome sequencing depth per sample) of the whole genomes of a large number of individuals. Here, we report exome sequencing of 200 individuals from Denmark with targeted capture of 18,654 coding genes and sequence coverage of each individual exome at an average depth of 12 -fold. On average, about $95 \%$ of the target regions were covered by at least one read. We identified 121,870 SNPs in the sample population, including 53,081 coding SNPs (cSNPs). Using a statistical method for SNP calling and an estimation of allelic frequencies based on our population data, we derived the allele frequency spectrum of cSNPs with a minor allele frequency greater than 0.02 . We identified a 1.8-fold excess of deleterious, non-syonomyous cSNPs over synonymous cSNPs in the low-frequency range (minor allele frequencies between $2 \%$ and $5 \%$ ). This excess was more pronounced for X-linked SNPs, suggesting that deleterious substitutions are primarily recessive. Based on our findings, we support the idea that much of the heritable variation affecting fitness is caused by low-frequency mutations, which are often overlooked in studies based on genotyping rather than resequencing. Further, we demonstrate that the use of next-generation sequencing is an important tool in population genetics studies.

\section{THE DNA METHYLOME OF HUMAN PERIPHERAL BLOOD MONONUCLEAR CELLS}

W. Liu, J. Li,Y. Li

Beijing Genomics Institute, Shenzhen, China

DNA methylation plays an important role in biological processes in human health and disease. Recent technological advances allow unbiased whole-genome DNA methylation (methylome) analysis to be carried out on human cells. Using whole-genome bisulfite sequencing at 24.7 -fold coverage (12.3-fold per strand), we report a comprehensive $(92.62 \%)$ methylome and analysis of the unique sequences in human peripheral blood mononuclear cells (PBMC) from the same Asian individual whose genome was deciphered in the YH project. PBMC constitute an important source for clinical blood tests world-wide. We found that $68.4 \%$ of $\mathrm{CpG}$ sites and, $0.2 \%$ of non-CpG sites were methylated, demonstrating that non-CpG cytosine methylation is minor in human PBMC. Analysis of the PBMC methylome revealed a rich epigenomic landscape for 20 distinct genomic features, including regulatory, protein-coding, non-coding, RNA-coding, and repeat sequences. Integration of our methylome data with the YH genome sequence enabled a first comprehensive assessment of allele-specific methylation (ASM) between the two haploid methylomes of any individual and allowed the iden- tification of 599 haploid differentially methylated regions (hDMRs) covering 287 genes. Of these, 76 genes had hDMRs within $2 \mathrm{~kb}$ of their transcriptional start sites of which $.80 \%$ displayed allele-specific expression (ASE). These data demonstrate that ASM is a recurrent phenomenon and is highly correlated with ASE in human PBMCs. Together with recently reported similar studies, our study provides a comprehensive resource for future epigenomic research and confirms new sequencing technology as a paradigm for largescale epigenomics studies.

\section{A NEW WAY TO RESEARCH INTO HUMAN GUT MICROBIOME}

W. Liu, J. Li, Y. Li

Beijing Genomics Institute at Shenzhen, Shenzhen, China

As our other genome, microbes living in human body are closely related with human health. To understand the impact of gut microbes on human health and wellbeing, it is crucial to assess their genetic potential. A human gut microbial gene catalogue has been established by using the Illumina-based metagenomic sequencing and assembly approach, which identified 3.3 million non-redundant microbial genes, derived from 576.7 giga bases of sequence, from fecal samples of 124 European individuals. The gene set, $\sim 150$ times larger than the human gene complement, contains an overwhelming majority of the prevalent (more frequent) microbial genes of the cohort and probably includes a large proportion of the prevalent human intestinal microbial genes. The genes are largely shared among individuals of the cohort (J.Qin et al., Nature 464, 59 (2010)). Using the gene catalogue as reference, we expect to find the correlation between some metabolic diseases and gut bacteria, dig into the evolution of bacteria community and explore repeat expansion. With modification of PCR reaction conditions it was possible to size the child's expansion to be $185 \mathrm{CAG}$ repeats $(+/-10$ repeats). These results demonstrate that very large expansions can occur through the maternal lineage if the mother carries an expansion of sufficient size, and that these expansions can be detected and sized by PCR analysis.

\section{GENETIC INVESTIGATIONS IN MULTIPLE SCLEROSIS: POLYMORPHIC INVESTIGATION WITHIN THE REGION OF SEVERAL NUCLEAR RECEPTOR GENES FOR ASSOCIATION WITH MULTIPLE SCLEROSIS WITHIN AN AUSTRALIAN CASE-CONTROL POPULATION.}

J. Mackenzie', L. Tajouri',A. Szvetko', J. Fowdar', J. Greer ${ }^{2,3}$, P. Csurhes ${ }^{2,3}$,

M. Pender ${ }^{2,3}$, L. Griffiths'

1 Genomics Research Centre, Griffith University, Southport, Australia

2 Neuroimmunology Research Centre, School of Medicine, University of Queensland, Australia

3 Department of Neurology, Royal Brisbane and Women's Hospital, Brisbane, Australia

Introduction: Multiple sclerosis (MS) is a degenerative central nervous system disease associated with the formation of demyelinated plaques. These plaques cause damage to the axonal insulator, myelin, leading to alteration of the action potential conduction along axons and results in broad and various symptoms. The distinct cause of the disease is still not completely clear and there is no cure to date. Studies indicate both the implication of genetic predisposition and unknown environmental factors in the disease. The aim of this research was to examine several key genetic factors of several nuclear receptors that may contribute, or predispose individuals, to the disease. Methods: The test groups consisted of 210 MS patients and 210 controls, matched for ethnicity, sex and age (+/- 5 years). Polymorphisms were genotyped using restriction fragment length polymorphism, high resolution melting and genotype sequencing, to detect each variant in the genes of interest. Statistical analysis of genotype and allele frequencies included Hardy-Weinberg law and conventional contingency table analysis incorporating the standard chi-squared test for independence. Results, Conclusions and Future Directions: The results indicate one of the polymorphisms may be associated with MS, therefore we are confirming our results at 
present with another marker. Results for the other markers indicate that the polymorphisms are not associated with MS, however we cannot exclude the hypothesis that other polymorphic alleles of the remaining candidate genes could be implicated in MS susceptibility. Therefore, we will be performing further investigations of the remaining candidate genes in the near future. Ultimately, this investigation may help pinpoint genes implicated in MS which may result in a greater understanding of some of the cellular mechanisms involved in MS pathology, new gene targets with therapeutic benefits or significant diagnostic benefits.

\section{EVIDENCE FOR A NOVEL MIGRAINE SUSCEPTIBILITY LOCUS ON CHROMOSOME X012 IN A LARGE PEDIGREE FROM THE GENETIC ISOLATE OF NORFOLK ISLAND}

B. H. Maher', H. C. Cox', L. M. Haupt', C. Bellis², J. Blangero'², J. Curran², R. Lea', L. R. Griffiths'

${ }^{1}$ Griffith Health Institute, Griffith University, Gold Coast, Australia

${ }^{2}$ Southwest Foundation of Biomedical Research, Texas, United States

Migraine is a common and debilitating neurovascular disease that has a complex envirogenomic etiology. Numerous studies have demonstrated a preponderance of women affected with migraine and previous pedigree linkage studies in our laboratory have identified susceptibility loci on chromosome Xq27 and 28. The present study continues this line of investigation by performing a complete $\mathrm{X}$ chromosome scan of a very large migraine pedigree from the genetically isolated population of Norfolk Island. A novel pedigree-based association approach incorporating logistic regression was employed to analyse $\mathrm{X}$ chromosome-wide SNP data $(15,154$ SNPs). Data was ascertained for $n=288$ related individuals comprising a large core-pedigree of isolated Norfolk Island population. This pedigree is comprised of 76 individuals affected with IHS-diagnosed migraine (MO $n=25$ and MA $n=51$ ). All SNPs were ranked based on P-values adjusted for the effects of relatedness, gender and age. SNP prioritization showed that the top 25 SNPs contained 13 SNPs localized to the previously identified loci on Xq27 and 28 adding further support to the involvement of these genomic regions in migraine susceptibility. Interestingly, 10 of the 25 top ranked SNPs mapped to a new $377 \mathrm{~Kb}$ locus at Xq12 - strongest association at rs670546 $P=$ $9.67 \times 10-5$. Haplotype analysis of this new region highlighted the presence of 2 major haplotype blocks each containing a haplotype that was significantly over-represented in the migraineurs compared to controls $(P<.0005)$. In conclusion, we have provided strong evidence supporting the presence of a novel migraine susceptibility locus on Xq12 in a very large pedigree from the isolated population of Norfolk Island. Investigations are now underway examining these Xq12 haplotypes in unrelated case-control populations to determine whether this locus is unique to Norfolk or influences migraine susceptibility more generally.

\section{OBSERVATION OF THE CANCER PATIENT JOURNEY:} A LEARNING CURVE FOR GENETIC COUNSELORS

\section{K. J. Mann, J.A. Taylor \\ Familial Cancer/Adult Genetics, Royal Melbourne Hospital, Melbourne, Australia}

Cancer genetic counselors typically work in hospitals and participate in multidisciplinary clinical teams. However, in their training, genetic counselors seldom have much exposure to the medical environments in which their clients receive their cancer treatment. Although there is a general understanding about screening, learning a diagnosis, surgical, chemotherapy, and radiotherapy treatments, many of the genetic counselors have no firsthand experience of these processes, making it difficult to comprehend the impact it has on clients. This project aimed to develop knowledge and awareness of what a cancer patient would experience throughout their cancer journey, therefore increasing the scope for empathy and consideration for clients seen. Second, this project aims for genetic counselors to gain a better appreciation of the risk management procedures and surgeries that enter into the genetic counselor's discussions with clients of a Familial Cancer Centre (FCC). Two genetic counselors approached several senior clinicians (including medical oncologists, gastroenterologists, surgeons, and gynecologists) affiliated with the Royal Melbourne Hospital FCC to observe both consultations and medical procedures in their speciality area. The genetic counselors reflected on their observations of the patient's cancer journey and how the patient's experiences may impact on a genetic counseling session. Both counselors gained a greater insight into the enormity of a cancer diagnosis on an individual. We wish to share our experience: a greater knowledge of the screening and surgical recommendations that are discussed in a genetic counseling session, an increased awareness of the role of other medical professionals in the patient's cancer journey and a better understanding of how the FCC integrates with the patient's cancer journey. This project warrants extension as we believe that by being better equipped to understand our patients and their needs, we can provide better patient care.

EARLY ONSET HUNTINGTON DISEASE PRESENTING AS A NEUROFERRITINOPATHY

\footnotetext{
C. S. Manning ${ }^{1,2}$, J. C. MacMillan ${ }^{1,2}$, M.T. Gabbett ${ }^{1,2}$

${ }^{1}$ Royal Brisbane \& Women's Hospital, Genetic Health Queensland, Brisbane, Australia

${ }^{2}$ School of Medicine, The University of Queensland, Brisbane, Australia
}

Neuroferritinopathy caused by mutations in the FTL gene causes gradual accumulation of iron in the brain. Clinically, this results in a progressive adult-onset chorea or dystonia and subtle cognitive defects. The majority of individuals develop a characteristic orofacial actionspecific dystonia that leads to dysarthrophonia. Cognitive deficits and behavioral issues become significant with time. Magnetic resonance imaging (MRI) typically demonstrates hypointensity of the basal ganglia on T2-weighted imaging. Huntington disease (HD) is a progressive disorder of motor, cognitive, and psychiatric disturbances and is caused by an expanded polyglutamine tract in the huntingtin protein. MRI typically demonstrates basal ganglia atrophy. We present the case of a 27-year-old Vietnamese woman with a 3-year history of symptoms including dysphagia, imbalance and low vision. Her father and her brother were said to be similarly affected. MRI demonstrated decreased T2 signal within the basal ganglia, dentate cerebellar nuclei and the substantia nigra, consistent with iron disposition. The clinical presentation and MRI findings suggested an autosomal dominant neuroferrintinopathy. Testing of the FTL gene yielded no mutations. Testing HD demonstrated CAG trinucleotide repeats of 16 and 57, consistent with the diagnosis of HD. Hypointensity of the basal ganglia represents iron deposition on T2-weighted MRI, but has rarely been reported in HD. This case illustrates that HD should be considered as a differential diagnosis for neuroferrtinopathy.

${ }^{1}$ American Journal of Neuroradiology 1987; 8:397-411.

${ }^{2}$ Journal of Neurology, Neurosurgery and Psychiatry 1993; 56:834-835.

\section{THE CHALLENGE OF FOLLOWING A LOW LYSINE DIET FOR CHILDREN WITH GLUTARIC ACIDURIA 1 - NOT FOR} EVERYONE

B. C. Martino, G. Knight, A. Martin

Princess Margaret Hospital, Perth, Australia

Purpose: To discuss the challenges two families face in providing a low lysine diet as dietary management of Glutaric aciduria 1. Introduction: In 2007 Kolker et al. ${ }^{1}$ released guidelines for the diagnosis and management of glutaryl-CoA dehydrogenase deficiency (Glutaric aciduria type I). An updated guideline was released in 2011. ${ }^{2}$ Although the mainstay of treatment for GA1, aggressive management of intercurrent illnesses is not debated, dietary management is not clear. Kolker et al. recommended a diet which restricted lysine and was supplemented with lysine-free, tryptophan-reduced amino acid mixtures. Method: Patient 1: The patient followed a low-protein diet that was supplemented with a lysine-free tryptophan-reduced amino acid mixture from birth until 12 months. On the basis of the newly released Kolker et al. (2007) recommendations, the patient was changed to a low lysine diet whilst still continuing with the amino acid mixture. Patients 2 and 3: These patients were twins born at 28 weeks 
gestation. They were commenced on a low-lysine diet and lysine-free tryptophan-reduced amino acid mixture from birth. Summary of Results: Due to the challenging nature of the diet, there was frequent communication between the dietitian and the mother of the children on an outpatient basis. Patient 1 has generally been able to meet her nutritional requirements within the lysine restriction. The parents now find that the low-lysine diet is easy to follow and they are able to "normalise' their child's diet more as she gets older and her total daily lysine requirement increases. Patients 2 and 3 have struggled to meet their nutritional requirements. Their parents have found the lysine restriction particularly onerous and restrictive. Consideration is being given to change to a counting protein rather than lysine for this family to make the diet easier to implement.

${ }^{1}$ Journal of Inherited Metabolic Disease 2007; 30:5-22.

${ }^{2}$ Journal of Inherited Metabolic Disease, 2011 (Published online 23 March).

\section{NAPHTHALENE EXPOSURE AND G6PD}

\section{A. C. McWhinney', F. Bowling ${ }^{2}$}

${ }^{1}$ Clinical Chemistry, Pathology, Mater Health Services, Brisbane, Australia

${ }^{2}$ Biochemical Diseases, Mater Health Services, Brisbane, Australia

We report an interesting case of a full-term baby of Burmese descent, discharged on day 1 of life with no jaundice and apparently well. On day 3 of life, parents presented the baby to Casualty with severe jaundice and a serum bilirubin (SBR) of $610 \mathrm{mmol} / \mathrm{L}$. Phototherapy was commenced and the SBR decreased to $450 \mathrm{mmol} / \mathrm{L}$ within 8 hours. Respiratory distress and seizures developed the next day, and the baby was transferred to Pediatric ICU. Investigations were commenced to determine the cause of the hyperbilirubinemia. The child was subsequently diagnosed as having glucose-6-phosphate dehydrogenase (G6PD) deficiency. Individuals with this disease may exhibit nonimmune hemolytic anemia in response to a number of causes, most commonly infection or exposure to certain medications or chemicals. Analysis of urinary organic acids showed the presence of naphthalene, which has been identified as a trigger for hemolytic anemia in patients with G6PD deficiency. Following treatment, bilirubin levels returned to normal levels 6 days after the exposure to naphthalene. The baby was subsequently transferred to the local community hospital. Investigations revealed that the child had been wrapped in blankets which had been stored with mothballs. It is postulated that due to the recent floods in Brisbane and the significant loss of property, blankets from long-term storage were made available. Naphthalene exposure has been identified as a significant health risk for children and a recent article has recommended that there be an Australia-wide ban of mothballs containing naphthalene. Subsequent to this case, the presence of naphthalene has been detected in another two routine organic acid screening profiles. This phenomenon has not previously been seen in our laboratory.

\section{'HANGING LIKE A SHADOW' - EXPLORING HEALTHCARE PROFESSIONALS' EXPERIENCES OF WORKING WITH TERMINATION OF PREGNANCY FOR FETAL ABNORMALITY}

M.A. Menezes ${ }^{1,2}$, J. Hodgson ${ }^{1,2}$, M. Sahhar ${ }^{2,3}$, S.A. Metcalfe ${ }^{1,2}$

${ }^{1}$ Genetics Education and Health Research, Murdoch Childrens Research Institute, Melbourne, Australia

${ }^{2}$ Department of Paediatrics, The University of Melbourne, Australia

${ }^{3}$ Genetic Health Services, Victorian Clinical Genetics Service, Melbourne, Australia

Pregnant women in Australia are increasingly offered an array of antenatal screening and diagnostic tests which provide health information about the developing fetus. This has consequently led to increases in the number of abnormalities that are identified and diagnosed during pregnancy. Women who receive a diagnosis of a fetal abnormality are offered the choice of either continuing or terminating their pregnancy, and are cared for throughout the diagnosis, decision-making, and thereafter by a range of healthcare professionals. The aim of this $\mathrm{PhD}$ research project was to explore whether working in the fetal medicine setting has any impact on healthcare professionals. 40 in-depth interviews were conducted with medical and allied healthcare professionals who work with couples receiving a diagnosis of a fetal abnormality. Interviews were audio-taped, transcribed verbatim and analyzed using thematic analysis. The provision of TOP services was described by participants as an implicit aspect of the fetal medicine setting, 'hanging like a shadow' over their consultations. Three main ethical and moral challenges associated with involvement in the provision of TOP services were highlighted by participants: (1) the complexities associated with counseling patients about decision-making around TOP, (2) difficulties in ensuring women have access to TOP for fetal abnormality as only select healthcare professionals and hospitals agree to provide the procedure, (3) personal moral dilemmas experienced by participants as a result of being involved in TOP, which is perceived to be 'dirty work'. These findings and their implications for practice will be discussed. In particular, there appears to be a need for a more open dialogue around the provision of TOP services in fetal medicine settings, and for institutional support for healthcare professionals working in this area of medicine.

\section{USING ENU MOUSE MODELS TO UNDERSTAND HUMAN} SKELETAL DYSMORPHOLOGIES

K. Miller', T.Tan I, C. Gordon', T. McMorran ',2, P. Farlie ${ }^{1,2}$

1 Craniofacial Research, Murdoch Childrens Research Institute, Melbourne, Australia

2 Royal Children's Hospital, Department of Paediatrics, Melbourne, Australia

Birth defects are structural or biochemical abnormalities that are present at birth, and are the leading cause of infant morbidity and mortality, affecting 3-5\% of live births in the United States. Approximately one third of all congenital abnormalities involve the craniofacial structures, where they are frequently associated with other clinical characteristics such as defects in the limbs and/or other organ systems. Mouse N-ethyl-N-nitrosourea (ENU) mutagenesis screens have proven to be an invaluable tool for the functional identification of novel disease genes, and to underpin our studies on the genetic control of morphogenesis and congenital malformation in humans we undertook a comprehensive recessive mutagenesis screen to identify novel mouse models of human dysmorphology. We have identified several mouse mutants with skeletal deformities. One mouse model, BLOOD, exhibits phenotypes including cryptothalmia, polydactyly and blood skin blisters. A mutation in the Fras1 gene was identified in this mutant where the phenotype is highly reminiscent of the 'blebs' mouse mutants and the human condition Fraser syndrome (OMIM 219000). Our CAULI strain is a likely model for the human conditions Jeune syndrome or asphyxiating thoracic dystrophy 1 (OMIM 208500), with phenotypes including exencephally, mandibular overgrowth, anophthalmia, hindlimb polydactyly and mixed polydactyly/oligodactyly of the forelimbs. KANYON mutant embryos have a mid-facial cleft and a variable mid-brain excencephaly, similar to a group of conditions known as frontonasal dysplasias (OMIM 136760). Characterization of these ENU mouse mutant strains will highlight the biochemical outcome of particular mutations and the fundamental mechanisms responsible for normal skeletal development that will facilitate the identification of underlying mutations in correlating human conditions.

\section{A TARGETED FLUORESCENCE IN SITU HYBRIDIZATION (FISH) PROCEDURE TO ASSIST IN DIFFERENTIATING BENIGN FROM MALIGNANT MELANOCYTIC LESIONS}

E. G. Baker', J. Peverall', A. R. Murch ${ }^{1,2}$,T.W. Beer ${ }^{3}$

${ }^{1}$ Cytogenetics Dept KEMH, PathWest, Perth, Australia

${ }^{2}$ Pathology and Laboratory Medicine, University of Western Australia, Australia

${ }^{3}$ Cutaneous Pathology, Perth, Australia

Pathology is the gold standard for the diagnosis of malignant melanoma. Most nevi and most melanomas can be accurately diagnosed from routine histopthological examination. There are, however, many histopathologic simulators of melanoma that pose problems. Over-diagnosis of melanoma can lead to inappropriate therapy and psychological burdens, and under-diagnosis can lead to 
inadequate treatment of a deadly cancer. To aid melanoma classification we evaluated a new Immuno FISH test that uses signal counts from a panel of 4 probes targeting RREB1 (6p25), CEP 6, MYB (6q23) and CCND1 (11q13) (Abbott Molecular Laboratories, USA). A scoring algorithm is applied to determine FISH positivity. The evaluation was performed on FFPE sections from 5 cases with normal skin, 10 cases with benign melanocytic nevi, and 10 cases with malignant melanoma.

\section{ENVIROGENOMIC RISK PROFILING TO PREDICT CLINICAL OUTCOMES IN CROHN'S DISEASE PATIENTS}

B. F. Nasir, R. Lea, R. Gearry, A. Nasir, D. Macartney-Coxsin, D. Hall, L. Griffiths Genomics Research Center, School of Medical Sciences, Griffith Institute of Health and Medical Research, Griffith University, Australia

Crohn's disease (CD) is a chronic inflammatory bowel disease characterized by severe abdominal pain. Being able to identify patients at high risk of complicated disease progression should assist clinicians to decide whether or not to prescribe early aggressive treatment with immunomodulators. Prediction of CD outcome is influenced by clinical, environmental, serological and genetic factors (e.g., NOD2). Our research aims to identify envirogenomic risk profiles for accurate prediction of complicated CD. Methods: (1) We re-analyzed the WTCCC data set for 550K SNPs in $1700 \mathrm{CD}$ cases using a novel Genomic Signature Analysis (GSA) algorithm; (2) We re-analyzed the Canterbury IBD patient cohort data set for $\sim 700 \mathrm{CD}$ patients including clinical and environmental factors using standard multifactor regression methods. Results: Using our GSA algorithm we discovered and validated a 42-SNP signature, including the NOD2 gene, which produces a risk score $(\mathrm{AUC}=0.7)$ and a rare genomic risk factor with a diagnostic accuracy of $80 \%(\mathrm{OR}=4, P=1 \times 10-10)$. Analysis of the Canterbury IBD data set revealed the best predictive model for complicated CD to contain 2 factors - NOD2 gene and perianal disease. The max. AUC for the model probabilities was $0.62(P<.001)$. Discussion: We have discovered a new genomic signature for predictive $\mathrm{CD}$, which may offer some diagnostic utility for identifying high-risk patients. We also show that envirogenomic modeling including the single gene (NOD2) yields only modest diagnostic accuracy. We now plan to type the new multi-SNP signature in the clinical cohort and utilize 'optimization' modeling procedures and more environmental factors (e.g., diet) to improve the overall accuracy. The goal of this research is to develop a simple clinical tool for accurate identification of high-risk Crohn's patients that can aid clinicians when making decisions about personalized treatment options for patients.

\section{RAPID PRENATAL ANEUPLOIDY DIAGNOSIS USING REAL-TIME PCR}

\section{Newbound, S. Chen, J. Beilby}

Molecular Genetics, PathWest Laboratory Medicine Western Australia, Perth, Australia

Introduction: Reliable rapid aneuploidy diagnosis (RAD) techniques are widely used for the early detection of Trisomy 13, 18 and 21 and sex chromosome aneuploidy by Quantitative Fluorescent PCR (QF-PCR) of short tandem repeats. This technique is rapid and accurate but limited by reliance on variation in allele fragment size for enumeration. TaqMan ${ }^{\circledR}$ copy number assays (CNA) provide a novel approach to determination of chromosome copy number via change in cycle threshold (CT) for a gene target, compared to endogenous control gene (delta delta CT method). Methods: CNAs were constructed using multiplexed VIC-labeled endogenous control primer/probe (i.e., RNaseP gene), coupled with FAM-labeled primer/probe for target gene; X-specific (AMELX, exon 6); or Y-specific (AMELY, exon 5) Amelogenin, amplified in triplicate. Twenty seven patient amniotic fluid samples were investigated for X and Y chromosome specific CNA, QF-PCR and karyotype results. Four commercially obtained cell lines (Coriell, New Jersey, USA) were used for validation purposes. Results: Results for 25 of the 27 samples were concordant for CNA, QF-PCR and karyotype. Of the 2 aberrant results, one was due to the CNA detecting 3 copy numbers of the $\mathrm{X}$ chromosome which were not identified by QF-PCR.
Karyotyping revealed a mosaic population of X/XXX. The other sample contained a high salt content which skewed the D D CT in CNA and overestimated the copy numbers. Conclusions: This pilot study determined that CNA can reliably detect and determine chromosomal copy number providing the DNA is of comparable quality. Results are available more rapidly than with QF-PCR. However, unlike QF-PCR, CNA did not produce any uninformative results and provides an excellent supplementary means of RAD.

\section{NEWBORN SCREENING FOR GUANIDINOACETATE} METHYL TRANSFERASE DEFICIENCY: A PILOT STUDY

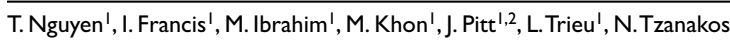

${ }^{1}$ Newborn Screening, VCGS, Melbourne, Australia

${ }^{2}$ Department of Paediatrics, University Of Melbourne, Australia

Guanidinoacetate methyl transferase deficiency (GAMTD) is an inborn error of creatine biosynthesis resulting in developmental delay, ataxia and seizures. Diagnostic indicators are decreased creatine and creatinine and increased guanidinoacetic (GAA) in urine and blood. We investigated the feasibility of newborn screening for this disorder via measurement of GAA in dried blood spots using an additional mass transition in the routine MSMS screening panel. Retrospective analysis of the newborn dried blood spot stored for 24 years from a positive case showed a clearly increased level of GAA (10.1 Multiple of Median (MoM), storage matched controls $<3.6 \mathrm{MoM}$ ). The actual level may have been even more pronounced because GAA levels appeared to increase with storage (fresh controls $<1.6 \mathrm{MoM}$ ). Prospectively, we have tested 673466 babies since 2002 without detecting a case. No false negative screening results have been revealed in the same cohort through clinical surveillance/urine screening of symptomatic patients. Many premature babies were found to have an apparently increased GAA level on repeat sampling due to an interfering substance. Full evaluation of the effectiveness of GAMTD newborn screening will require the prospective diagnosis of cases. Our pilot study suggests that GAMTD is quite rare in the Victorian population but its inclusion in the newborn screening panel is easily implemented with a low false positive rate.

\section{ADHERENCE AND TRANSITION IN METABOLIC DISEASE: AN ADULT WITH CLASSICAL HOMOCYSTINURIA AND MULTIPLE THROMBOSES}

\section{R. Nicol, C.Wilson}

Metabolic Services, ADHB, Auckland, New Zealand

Adherence describes an individual's commitment to a given treatment plan. Challenges arise when there is insufficient knowledge about the disease or the consequences of non-adherence, financial constraints, poor palatability or high frequency of treatment, and the most vulnerable time for poor adherence to occur is during periods of transition. Treatments for inborn errors of metabolism are frequently lifelong, complex and demanding and non-adherence can have devastating consequences. Accordingly, parents are frequently key players in ensuring adherence even into adulthood. Effective transition from pediatric care to adult services primarily aims to ensure that the adolescent is protected whilst maintaining their autonomy so that they are equipped with the skills to maintain continued adherence into adult life. The case presented to demonstrate these concepts is a 26-year-old male with classical homocystinuria. Throughout childhood and adolescence his diet and medication were managed primarily by his parents. Compliance was excellent, reflected by the excellent metabolic control and no significant complications from his disease. At 18 years of age, however, he left home and took on responsibility for his treatment. With this came challenges of adherence. Subsequent poor compliance with diet, betaine and anti-coagulation therapy has lead to multiple admissions to hospital with life and limb threatening thromboses. Each time he stated it would be the last and he would turn his life around. However, he continues to struggle with the treatment regimen and monitoring plan. Strategies put in place to support his commitment 
to resume compliance with the ultimate goal to have him take ownership and control over his disease will be discussed.

\section{SCREENING FOR CONGENITAL HYPOTHYROIDISM IN LOW BIRTHWEIGHT BABIES: QUEENSLAND NEWBORN SCREENING DATA}

\section{O'Brien, A.Thomas, J. Ungerer}

Chemical Pathology, Pathology Queensland, Brisbane, Australia

Newborn screening for congenital hypothyroidism relies on the maturity of the hypothalamic-pituitary-thyroid axis to reveal an elevated level of thyroid stimulating hormone (TSH). Low (between 1000 and $1500 \mathrm{~g})$ and very low $(<1000 \mathrm{~g})$ birthweight babies can show a delay in the rise of TSH after birth resulting in false negative screening results. ${ }^{1}$ This is primarily due to immaturity of the axis. The Australasian Paediatric Endocrine Group recommends that TSH screening should be repeated in low birthweight babies at 2 weeks after birth and at 4 weeks in very low birthweight babies. ${ }^{2}$ In Queensland, these recommendations are outlined in the Newborn Screening Program 2008 Guidelines. For many Queensland hospitals it is routine practice to repeat the TSH screen at the recommended times but there is still debate in the literature about whether this is necessary. A recent study in NSW has supported the need for a second test so that very low birthweight babies who require thyroxine treatment are not missed. ${ }^{3}$ Our aim was to investigate the numbers of low and very low birthweight babies for whom we received a repeat screening sample at the Queensland Newborn Screening lab between 2007 and 2009. The TSH results for these samples were assessed to identify babies with abnormal results in the second screen. The results including compliance, diagnosis and followup treatment, will be presented and discussed.

${ }^{1}$ Lancet 1994; 343:60-61.

${ }^{2}$ Australasian Paediatric Endocrine Group (1999). Guidelines for management of congenital hypothyroidism. http://www.ape.g.org.au.

3 Journal of Inherited Metabolic Disorders 2011 (Published online 19 February).

\section{POOR ACCESS TO HEALTH CARE, HIGH COMPLICATION RATES AND LIMITED DISEASE KNOWLEDGE IN YOUNG AUSTRALIAN ADULTS WITH NF1.}

\author{
E. C. Oates $^{1,2}$, H. Crawford ${ }^{1,3}$, J. M. Payne ${ }^{1,2}$, K. N. North ${ }^{1,2}$ \\ ${ }^{1}$ Neurogenetics Department, Institute for Neuroscience and Muscle Research, \\ Kids Research Institute, Sydney, Australia \\ ${ }^{2}$ Discipline of Paediatrics and Child Health, University of Sydney, Australia \\ ${ }^{3}$ University of Ulster, United Kingdom
}

Neurofibromatosis type 1 (NF1) is a multisystem disease associated with a lifelong risk of debilitating, and potentially life-limiting complications, however many adults with NF1 have no regular health surveillance. We interviewed and examined 17 young adults with NF1 between the ages of 25 and 33. Most cohort members had not been assessed for NF1-related complications within the previous eight years, including patients with known serious vascular complications, for example, renal artery stenosis. Acute and/or chronic pain, particularly back pain, was common, and despite a significant impact on quality of life, was untreated in most instances. Symptom and examinationdirected imaging revealed serious complications in $40 \%$ of the cohort. These included severe cord compression (2 cases), highly-SUV avid lesions on PET scans suggestive of malignancy ( 2 cases), and a juvenile pilocytic astrocytoma. Few study participants had a good understanding of NF1 and its associated risks and complications. Most had not sought appropriate medical advice as questions or problems arose. Cognitive deficits in participants and lack of a clear source of expert medical advice for adults with NF1 likely contributed to poor access to health care. These findings suggest that many Australian adults with NF1 'at risk' of serious and life-threatening medical complications are not currently being managed according to internationally recognized standards of care. Access to multidisciplinary adult clinics that specialize in NF1 may address many of the unmet health needs of young adults with NF1.
THE PATHOLOGICAL BASIS OF AUTOSOMAL DOMINANT CONGENITAL SPINAL MUSCULAR ATROPHY

E. C. Oates ${ }^{1,2}$, N. F. Clarke ${ }^{1,2}$, S. Reddel ${ }^{2,3}$, M. L. Rodriguez ${ }^{4}$, S. H. Hawke ${ }^{5}$, K. N. North ${ }^{1,2}$

1 Neurogenetics Department, Institute for Neuroscience and Muscle Research,

Kids Research Institute, Sydney, Australia

2 Discipline of Paediatric and Child Health, University of Sydney, Australia

3 Departments of Neurology and Molecular Medicine, Concord Repatriation General Hospital, Sydney, Australia

4 Department of Forensic Medicine, Sydney South West Area Health Service, Sydney, Australia

5 Brain and Mind Institute, University of Sydney, Australia

'Classical' type 1 spinal muscular atrophy (SMA) is characterized by progressive muscle weakness caused by ongoing degeneration of anterior horn cells. Clinical progression after birth is universal. It has been postulated that rarer non-progressive forms of SMA result from abnormal fetal development and/or early (fetal) loss of anterior horn cells, without ongoing postnatal cell loss. We describe a four generation family in which six family members have non-progressive distal SMA that follows clear autosomal dominant (AD) inheritance. Most affected family members have mild weakness, limited to the lower limbs, with a history congenital talipes with or without congenital hip dysplasia. All remain ambulant. Features seen on EMG and muscle biopsy suggest an overall reduction in the number of motor units. Nerve conduction velocities are normal. On muscle MRI there is atrophy of most distal leg muscles, with preservation of the anterior compartment, and mild hypertrophy of the adductor longus and semitendinosus, a pattern that has been previously reported in distal SMA. A detailed post-mortem study of a 14-month-old affected girl from this family, who died from meningococcal meningitis, provides rare insight into the pathological basis of this condition. There was a paucity of anterior horn cells, in the absence of signs of active cell degeneration, which supports the hypothesis that the loss of anterior horn cells is limited to early development. Analysis of linkage to $12 \mathrm{q} 23-\mathrm{q} 24$, and other loci previously linked to AD congenital SMA is in progress.

\section{HEPARAN SULFATE PROTEOGLYCANS IN BREAST}

\section{CANCER}

R. K. Okolicsanyi, L. R. Griffiths, L. M. Haupt

Genomics Research Centre, Griffith University, Southport, Australia

Heparan sulfate proteoglycans (HSPGs) are members of the proteoglycan (PG) family of macromolecules that structurally and functionally influence cell proliferation, differentiation and gene expression. HSPGs are ubiquitous to the cell surface and the extracellular matrix (ECM), and are composed of a core protein to which one or more glycosaminoglycan (GAG) side chains attach. HS chains have been demonstrated to interact with a large number of ligands including growth factors and morphogens (FGF, Wnts, BMPs), their receptors (FGFRs), and extracellular matrix structural molecules (collagen, fibronectin). The HS chains are highly complex molecules with this complexity determined by both chain length and the chain sulfation pattern. Different sulfation patterns have been related to specific roles in differentiation and transformation of cells in cell culture. In this study, we examined the effect of HS, through its analogue heaprin, on the expression profile of HSPG core proteins and modifying enzymes for their involvement in human breast cancer initiation and progression. The human breast cancer cell lines, MDA-MB-231 (highly invasive, highly metastatic) and MCF-7 (poorly invasive, lowly metastatic) were examined at Days 1, 3 and 5 when grown alone and in the presence of heparin $(1,5,10,25$, and $50 \mathrm{ug} / \mathrm{mL}$ ). Initial results in MDA-MB-231 cells demonstrated a dose-response effect of heparin in terms of proliferation, specifically an increase in proliferative potential at Day 3 with a heparin dose of $10 \mathrm{ug} / \mathrm{mL}$. We then examined the gene expression profile of HSPG family members in these two cell lines at these time points by QPCR. The HSPG sepecific genes included those involved in chain initiation, polymerization, sulfation regulating enzymes as well as 
those responsible for core protein production (syndecans, glypicans etc) and the HSPG specific ECM remodeling enzyme, heparanase.

\section{ESTABLISHMENT OF A DIAGNOSTIC SERVICE FOR ALAGILLE SYNDROME. \\ E. Ormshaw', G. Jenkins', M. Brett', M. Mihelec', L. St Heaps², J. Christodoulou ${ }^{3,4,5}$, B. Bennetts $1,4,5$ \\ ${ }^{I}$ Department of Molecular Genetics, The Children's Hospital at Westmead, Sydney, Australia \\ ${ }^{2}$ Department of Cytogenetics, The Children's Hospital at Westmead, Sydney, Australia \\ ${ }^{3}$ The Genetic Metabolic Disorders Service, The Children's Hospital at Westmead, Sydney, Australia \\ ${ }^{4}$ Discipline of Paediatrics \& Child Health, The University of Sydney, Australia \\ ${ }^{5}$ Discipline of Genetic Medicine, The University of Sydney, Australia}

Alagille syndrome (AGS, MIM\# 118450) is an autosomal dominant, congenital malformation disorder affecting mainly the liver, heart, skeleton, eye and face. Minor features involving kidney, arteries, ear, and limb have also been described. AGS is caused by heterozygous mutations in Jagged1 (JAG1), a ligand in the Notch signaling pathway. JAG1 contains 26 exons; mutations are spread throughout the gene with no apparent mutational hotspots. Approximately 5-7\% of individuals have a microdeletion of 20p12, including the entire JAG1 gene which can be detected by FISH. JAG1 testing at The Children's Hospital, Westmead (CHW) was originally set up as part of an honours project in 2003. FISH using clone RP5-1099D15 was performed on all CHW patients in the first instance to detect for whole gene deletions. Denaturing high performance liquid chromatography (dHPLC) of the entire JAG1 coding region was then performed and any variants detected were characterized by direct sequencing. The project was moved over to the CHW diagnostic laboratory and gradually moved over to bidirectional sequencing of all exons. It has recently been further developed to ensure that all amplicons amplify at the same PCR conditions and by the addition of M13 tags onto all primers to increase efficiency. This has led to a reduction in turnaround times, since one patient can now be screened for all exons at one time. We are currently investigating the feasibility of the inclusion of MLPA into the current screening strategy. To date we have screened a total of 61 patients with pathogenic mutations being detected in 26 patients. Of the 26 mutations, $81 \%$ were protein truncating, $11 \%$ missense and $8 \%$ were splice site mutations.

\section{IS ACTIVATED AT THE CELL CYCLE CHECKPOINT IN RESPONSE TO GENOTOXIC STRESS}

L. Pangon, N. Sigglekow, M. Larance, E. Musgrove, M. Kohonen-Corish

Garven Institute of Medical Research, Sydney, Australia

The 'Mutated in Colorectal Cancer' (MCC) gene was discovered in 1991 due to its close linkage with the APC tumor suppressor gene. We have shown that the MCC defect is more common in colorectal cancer than previously thought, as this gene is frequently silenced through promoter hypermethylation. Furthermore, mutated MCC is a 'driver' of carcinogenesis in a mouse model of colorectal cancer. Using mass spectrometry we identified two highly conserved ATM/ATR consensus phosphosites in the MCC protein. This suggested that MCC may participate in the cellular process that responds to DNA damage. We then tested this response by exposing cells to various DNA damaging agents. Ultraviolet radiation, but not phleomycin, caused PI3K-dependent phosphorylation of MCC and its localization to the nucleus. Re-expression of MCC in HCT15 cells led to a G2/M arrest, and MCC knockdown in HCT116 cells impaired the induction of a G2/M arrest following UV radiation. Finally, mutation of S118/120 to alanine impaired the G2/M checkpoint activity of MCC. These results suggest that MCC activity is required in the full induction of cell cycle arrest in the G2/M phase upon UV-induced single strand DNA breaks. Single strand DNA breaks are common in all cells, including the colon, and are caused by spontaneous DNA decay or reactive oxygen species. The ability to sense and respond to DNA damage is critical to the maintenance of genomic stability and the prevention of cancer and this could be one of the mechanisms whereby loss of MCC expression promotes colorectal carcinogenesis.

\section{NEW COMPLEXITIES TO CONSIDER IN LQT SYNDROME} GENETIC TESTING

S. Pantaleo, D. Du Sart, D. Phelan

Molecular Genetics, VCGS Pathology, Melbourne, Australia

Long QT-syndrome (LQTS) is a genetic cardiac channelopathy characterized by a prolonged QT interval on electrocardiogram, syncope and an increased risk of sudden death. To date, 12 genes have been associated with LQTS. For routine genetic testing, it is recommended to screen the 6 genes which are associated with the majority of cases, KCNQ1, KCNH2, SCN5A, KCNE1, KCNE2 and KCNJ2. The molecular genetics laboratory at VCGS Pathology has been offering sequencing and MLPA for the entire coding sequences of these genes. When RNA or protein products of genes are analyzed, it is not unusual to identify a number of different transcripts or isoforms of genes. This may be indicative of tissue specific splicing, relating to tissue specific functions or normal splice variants, which are not usually associated with clinical disease. New studies have been published in which evidence has been presented of alternative isoforms of the proteins encoded by KCNQ1, KCNH2 and SCN5A being expressed in the heart. The protein expressed by KCNH2, hERG, is now known to be alternatively transcribed into two subunits, $1 \mathrm{a}$ and $1 \mathrm{~b}$. These two proteins form a heteromer which conducts $80 \%$ more charge during an action potential than 1a alone, due to differences in their $\mathrm{N}$ termini. A mutation in a $\mathrm{KCNH} 2$ isoform (encoding for the $1 \mathrm{~b}$ subunit) has since been described (p.A8V) in a patient affected with LQTS who was previously genotypenegative. We are currently screening for mutations within the alternative transcripts in our LQT patient cohort. To date we have identified two patients with LQT, who have pathogenic mutations in the alternative transcript of $\mathrm{KCNH} 2$ and no pathogenic mutations in the most commonly expressed transcripts. Therefore, it is essential that testing for mutations within the LQT genes include screening not only for the most commonly expressed transcripts, but also for these alternate transcripts.

\section{A QUALITATIVE ANALYSIS OF A GENETIC COUNSELING STRATEGY TO FACILITATE COMMUNICATION WITHIN FAMILIES \\ J. Paul ${ }^{1,2,3}$, J. Hodgson ${ }^{1,2}$, C. Gaff ${ }^{4,5}$, J. Halliday ${ }^{2,6}$, S. Metcalfe ${ }^{1,2}$ \\ ${ }^{1}$ Genetics Education and Health Research, Murdoch Childrens Research Institute, Melbourne, Australia \\ ${ }^{2}$ Paediatrics, The University of Melbourne, Australia \\ ${ }^{3}$ School of Languages and Linguistics, The University of Melbourne, Australia ${ }^{4}$ Clinical Genetics Research, Murdoch Childrens Research Institute, Melbourne, Australia \\ ${ }^{5}$ Medicine, The University of Melbourne, Australia \\ ${ }^{6}$ Public Health Genetics, Murdoch Childrens Research Institute, Melbourne, Australia}

The Communication of Genetic Information in Families (GIF) project, currently underway in Victoria, is one of the first randomized controlled trials in genetic counseling. We developed a client-centered genetic counseling strategy to facilitate family communication of genetic information, delivered by telephone, complementing initial face-to-face consultations. Iterative development of the GIF strategy was informed by empirical research about family communication. Counseling and communication theories were incorporated to optimise clinical feasibility and maintain congruence with the tenets of genetic counseling. This BSc Honours project aimed to audit and refine the pilot counseling strategy. A qualitative approach was selected to capture the complexities of telephone calls with 8 clients that were audio-recorded, transcribed verbatim and fully de-identified. A multilayered analytical approach utilized content, thematic, and discourse analyses. Results demonstrated that the GIF protocol was easily adhered to and was congruent with genetic counseling practice and a client-centered approach. Results also highlighted the value in supporting individuals in communicating genetic information to their family members. The client-centered approach appeared to enhance 
participant empowerment in family communication and resulted in an overall positive interaction with the counselor. Findings have shown that this counseling strategy can be carried out successfully with this group of participants. As a result of this work, the GIF protocol was refined and has now been successfully implemented in 54 telephone counseling sessions. This analytical approach shows great utility for exploring genetic counseling practice. Given the lack of process studies in genetic counseling, further analysis of practice with this level of detail would be of value to the profession.

\section{IDENTIFICATION AND CHARACTERIZATION OF NOVEL CARDIOMYOPATHY GENES \\ D. Phelan', E. Fitzpatrick', H. Mountford', K. Pope', M. Bahlo ${ }^{3}$, P. Hickey ${ }^{3}$, C. Bromhead ${ }^{3}$, D. Du Sart ${ }^{5}$, P. James ${ }^{5}$, M. Delatyki ${ }^{1,2,4}$, R. Leventer ${ }^{6}$, D. Amor ${ }^{2,5}$, P. J. Lockhart ${ }^{1,2}$ \\ ${ }^{I}$ Bruce Lefroy Center for Genetic Health Research, Murdoch Childrens Research Institute, Melbourne, Australia \\ ${ }^{2}$ Department of Paediatrics, The University of Melbourne, Australia \\ ${ }^{3}$ Bioinformatics Divisions, The Walter and Eliza Hall Institute, Melbourne, Australia \\ ${ }^{4}$ Clinical Genetics, Austin Health, Melbourne, Australia \\ ${ }^{5}$ Victorian Clinical Genetics Service, Murdoch Childrens Research Institute, Melbourne, Australia \\ ${ }^{6}$ Department of Neurology, Royal Childrens Hospital, Melbourne, Australia}

The aim of the Accelerated Gene Identification Program (AGIP) is to utilize new genetic technologies to identify and subsequently characterise novel genes for genetic disorders. The program combines a clinical/research pipeline, whereby individuals and families with genetic disorders are identified and characterized by clinical genetic services. Samples are then directed to the laboratory at the Murdoch Children's Research Institute (MCRI) for linkage analysis and/or gene discovery using high density Single Nucleotide Polymorphism (SNP) arrays and Next Generation sequencing technologies. AGIP has recently identified and clinically characterized two families displaying distinct cardiac phenotypes. Family 1 presents with a syndromic form of hypertrophic cardiomyopathy in which the heart disease is accompanied by multiple pterygia (webbing). Family 2 has three members affected by a cardiomyopathy syndrome that varies from a lethal hypoplastic left ventricle malformation, to dilated cardiomyopathy with left ventricular non-compaction, to a pure form of dilated cardiomyopathy. Copy number analysis and linkage studies were performed using data from high density SNP-array analysis. The causative loci was mapped in both families to regions of the genome not previously associated with cardiomyopathy. In family 1 , the disease causing gene was linked to chromosome 4p16 (LOD score $=$ 2.9 , odds 807:1 in favor of linkage), and in family 2 linkage was identified to chromosome 15q25 (LOD score $=2.1$, odds 121:1 in favor of linkage). Both families are currently undergoing whole exome sequence analysis and future studies will investigate the function of the mutant protein in cell and animal models to determine the underlying pathogenic mechanism(s). The contribution of the genes to cardiomyopathy will also be determined by analysis of well-characterized patient cohorts. This research will lead to increased understanding of the molecular basis of cardiomyopathy and ultimately result in improved clinical outcomes for affected individuals and families.

\section{CEREBROTENDINOUS XANTHOMATOSIS: A TREATABLE} DISORDER DETECTED BY URINE MSMS SCREENING

\footnotetext{
J. J. Pitt ${ }^{1,2}$, A. Boneh ${ }^{1,2}$, M. Cardamone', M. Eggington', A. Mishra', M.Van Werkhoven'

${ }^{1}$ Victorian Clinical Genetics Service, Murdoch Childrens Research Institute, Melbourne, Australia

${ }^{2}$ Department of Paediatrics, University of Melbourne, Australia
}

Cerebrotendinous xanthomatosis (CTX) is caused by sterol 27-hydroxylase deficiency, resulting in abnormal bile acid biosynthesis. Patients are often diagnosed as adolescents or adults with progressive neurological deterioration and/or tendon xanthomata. Many patients have a prior history of less specific symptoms such as prolonged neonatal jaundice, cataracts or chronic diarrhoea in infancy or childhood. The diagnosis can be made by detecting increased serum cholestanol or abnormal urine bile alcohols. Chenodeoxycholic and statin treatment is effective in halting the progression of many of the symptoms. We describe our prospective experience screening for CTX since 2004 in urine samples submitted for metabolic screening from Victoria and Tasmania. Qualitative testing for cholestanepentol glucuronide (CPG) was performed as part of a broad inborn error panel using MSMS. Two unrelated patients were diagnosed with CTX from $~ 16,000$ samples. Patient A presented with autistic features, developmental delay and diarrhoea at 3 years while patient B presented with neurological symptoms at 48 years. CTX was not suspected at the time of the initial screening request. CPG levels were grossly increased in both patients (multiple of median values: 91 and 95 respectively, 99th centile <11.6) by screening and confirmed by bile alcohol profiling which also showed the characteristic pattern of increased cholestanetetrol and cholestanehexol glucuronides. Patient A was treated with chenodeoxycholic acid and Atorvastatin. His chronic diarrhoea has completely subsided within 2-3 months. It is too early to determine the effect of treatment on his autistic features and development. Urine screening for CTX is easily implemented as part of wider urine MSMS screening and has a high positive predictive value. The early diagnosis and treatment of patients such as A has obvious benefits in delaying or preventing the onset of irreversible neurological symptoms.

\section{THE INSIGHT DATABASE OF MISMATCH REPAIR GENE VARIANTS AND PROGRESS IN VUS CLASSIFICATION}

J. Plazzer, F. Macrae

The Royal Melbourne Hospital, Melbourne, Australia

The International Society for Gastrointestinal Hereditary Tumors (InSiGHT) coordinates efforts to reduce the burden of inherited colorectal cancer for patients worldwide. InSiGHT maintains a public database of mismatch repair gene variants - the result of an international effort to collect and curate all variants associated with Lynch syndrome. The ongoing curation and development of the database is also a pilot project of the Human Variome Project (HVP), with collaboration and linkage to the Australian Node of the HVP. InSiGHT is currently developing a 5-class system for the clinical interpretation of variants of uncertain significance (VUS). An international group of experts in Lynch syndrome (the InSiGHT Interpretation Committee) will have regular teleconferences to discuss and classify variants using a set of qualitative rules. Information selected from the database will be used to classify VUS and a detailed explanation for the classification will be made available. Simultaneously, submission of variant, tumor and de-identified patient/family information is encouraged to facilitate classification and expand the database contents. Additionally, work is progressing to develop a quantitative classification system. The InSiGHT database stores variant information from patients, literature, functional assays and other sources and encourages further submissions from labs and clinics.

\section{WELCOME TO THE RBWH CARDIAC GENETICS CLINIC}

G. Poke, L. O’Brien, J. McGaughran

Genetic Health Queensland, Royal Brisbane and Women's Hospital, Brisbane, Australia

Sudden cardiac death is a devastating event. In the 5 years to April 2011, members of 173 different families have attended the RBWH combined cardiac genetics clinic, many of whom have lost a relative unexpectedly. The process of making a diagnosis after a sudden unexpected death is a challenging one. There are a number of diagnoses to consider and often no answer is found. Family members, already distressed by their loss, can have their anxiety compounded by long waiting times for appointments and genetic results. Test results can be difficult to interpret, and at-risk family members may be hard to contact. We present a case of sudden unexpected cardiac death in a young woman, the issues faced by her relatives, and the ways they are addressed in the cardiac genetics clinic. Though the case presented is hypothetical, the questions asked by the family members are very real. 


\section{IDENTIFICATION OF THE MITOCHONDRIAL 13513G>A MUTATION IN A PATIENT WITH FOCAL SEGMENTAL GLOMERULOSCLEROSIS}

\author{
S. Rajagopalan, B. Hanna \\ Department of Clinical Genetics, Liverpool Hospital, Sydney, Australia
}

Mutations in mitochondrial DNA may present with multi-organ dysfunction with prominent neurological features, or its effects may be confined to a single organ. Mutations in the mitochondrial ND5 gene which encodes a subunit of Complex I of the respiratory chain, are a frequent cause of oxidative phosphorylation disease. The $13513 \mathrm{G}>\mathrm{A}$ mutation is a common ND5 gene mutation, which may lead to debilitating neurological diseases such as Leigh syndrome or MELAS (mitochondrial encephalomyopathy with lactic acidosis and strokelike episodes). This mutation may also cause Leber hereditary optic neuropathy (LHON) and milder mitochondrial phenotypes. We describe a 31-year-old lady with a mild intellectual disability, deafness, blindness and renal failure, who was found to carry the $13513 \mathrm{G}>\mathrm{A}$ mutation. She presented at age 24 years with unexplained renal impairment. Renal biopsy confirmed focal segmental glomerulosclerosis (FSGS). By age 28, she developed end-stage renal failure requiring hemodialysis. At age 25 years, she presented with rapidonset bilateral blindness. Ophthalmological examination was consistent with LHON. The $13513 \mathrm{G}>\mathrm{A}$ mutation was identified in a small proportion of her blood mitochondria. There was no relevant family history and the mutation was absent in her mother's blood and urine mtDNA. The $13513 \mathrm{G}>\mathrm{A}$ mutation is different from other more common mitochondrial DNA mutations, in that low mutant loads may cause significant dysfunction of the respiratory chain, leading to Leigh syndrome, MELAS, or milder phenotypes. The mutation is identifiable in blood and/or urine samples in most reported individuals, thus sparing the need for muscle or skin biopsies. This mutation is not commonly associated with renal impairment and has never been reported in an individual with FSGS. Therefore, we report the first patient, to our knowledge, with a histologically confirmed glomerulopathy who harbours the $13513 \mathrm{G}>\mathrm{A}$ mutation.

\section{GENE AMPLIFICATION IN ACUTE LEUKEMIA}

\section{S. Riyat, C. McCarthy}

Cytogenetics, Pathology Queensland, Brisbane, Australia

Gene amplification is an aberrant genetic event that leads to inappropriate activation of gene or oncogene expression, which plays a vital role in malignant transformation, fast progression of tumor cells and other cellular processes including drug resistance. Cytogenetically, gene amplifications manifest intrachromosomally as homogeneously staining regions, tandem segmental duplications, ring chromosomes and marker chromosomes or extrachromosomally as double minutes and submicroscopic episomes. It is a frequent genetic event in solid tumors, however a rare occurrence in hematological malignancies. In acute leukemia, it is most commonly associated with amplification of the MYC, MLL and RUNX1 genes. Increased copy number changes due to genomic amplifications are generally associated with aggressive disease and poor prognostic outcome. Therefore, the identification of amplified genomic segments is imperative in risk assessment and treatment stratification. We report clinical, cytogenetic and prognostic findings of five cases presented with acute leukemia involving different genes, and sites of genomic amplification ascertained using fluorescence in situ hybridization.

\section{CHANGING GEARS: DEALING WITH CHALLENGING}

\section{FAMILIES}

J. Robinson

Department of Medical Genetics, Sydney Children's Hospital, Sydney, Australia

An experienced practitioner will perform many aspects of their job as second nature. When you find something that works, you stick to it.
However, occasionally you encounter a situation that forces you to reevaluate your practice. Educating families is a large part of my role as the Genetics/Metabolic Clinical Nurse Consultant at Sydney Children's Hospital and it is something that I enjoy and believed that I was proficient at. Meeting a family with a child living with a metabolic condition who struggled to meet and understand their child's complex health needs was an opportunity to evaluate my skills as an educator and resource for the family. I needed to forget about how I had previously educated families and develop new strategies and methods to engage with this family. This included going back to basics and getting rid of all assumptions that I previously held. This poster will describe my journey with this family and detail the methods used to help engage the family in the health care of their child. It is an ongoing work in progress.

\section{DUPLICATION/DELETION IN CHROMOSOME 13 ARISING FROM A MATERNAL PARACENTRIC INVERSION}

B. Ross', J.Anderson', M. Gattas' , J. Harraway', B. Lundie '

${ }^{1}$ Cytogenetics, Sullivan Nicolaides Pathology, Brisbane, Australia

${ }^{2}$ Brisbane Genetics, Brisbane, Australia

Paracentric inversions are generally considered innocuous, and when a presumed paracentric inversion is inherited in an unbalanced form, there is a suspicion that the rearrangement may be an insertion, rather than an inversion. We present a case of a 4-year-old male with developmental delay. Initial investigation by conventional cytogenetics, based on dysmorphic features and poor feeding at birth, revealed what appeared to be a paracentric inversion of 13 q21.2-q22. Parental chromosome studies were requested, and the presumed inversion was also found to be present in the proband's mother. More recently, based on the suspicion that a small duplication, deletion or gene disruption at the breakpoints may have caused the proband's phenotype, an array CGH study was requested. This study surprisingly revealed a $15.7 \mathrm{mb}$ deletion at $13 \mathrm{q} 21.31 \mathrm{-q} 31.1$ and a $15.7 \mathrm{mb}$ tandem inverted duplication at 13q31.1-q32.1. Molecular investigation of the proband's mother showed no genetic imbalance, and by using sequential FISH probes within and immediately adjacent to the inverted segment, the presence of a paracentric inversion was confirmed, and the orientation of the duplicated/deleted segments in the proband was elucidated. The imbalance observed in the proband appears to be the result of a rare U-type recombination event within the paracentric inversion during maternal meiosis. Retrospective examination of the karyotypes of mother and proband revealed a slight difference in banding pattern that had not initially been detected.

\section{TWO NOVEL MUTATIONS IN THE NOTCH3 GENE}

B. Roy, N. Maksemous, R. Smith, G. Davies, L. Griffiths

Health, Genomics Research Centre, Parklands Drive, Australia

Background: Cerebral autosomal dominant arteriopathy with subcortical infarcts and leukoencephalopathy (CADASIL) is a hereditary disease of small vessel, caused by mutations in the NOTCH3 gene ${ }^{1,2}$, located on chromosome 19p12. The disease generally occurs in adults and is characterized by repeated subcortical infarctions, dementia and also migraine with aura. ${ }^{3,4}$ NOTCH3 is a single-pass transmembrane receptor which has 34 tandem epidermal growth factor-like (EGF-like) repeats. The gene consists of 33 exons which encode a protein of 2321 amino acids. ${ }^{5}$ Exons 3 and 4 have been found to be mutation hotspots, containing more than $65 \%$ of all CADASIL mutations. ${ }^{5,6}$ Methods: We performed diagnostic CADASIL testing involving mutation and polymorphism screening in exons 2, 3, 4, 11, 18 and 19 in NOTCH3. DNA was extracted from patient blood (QIAamp, Qiagen) and the exons were amplified by PCR. PCR products were then purified, and sequencing reactions were carried out for the sense and antisense strands using Big Dye Terminator chemistry on ABI3130 Genetic Analyser. Sequence results were analyzed using Chromas and compared with the NCBI reference sequence. Results: Here we report two novel non-synonymous mutations identified in the NOTCH3 gene. Firstly, we report an alanine to valine amino acid 
change at a.a. $202(590 \mathrm{C}>\mathrm{T})$, located in exon 4, found in a 49-year-old female. Second, we report a cysteine to arginine amino acid change at a.a. $579(1735 \mathrm{~T}>\mathrm{C})$, located in exon 11 , found in a 66 year-old female. Conclusion: Although Ala202Ala (rs1043994) is a common polymorphism in the NOTCH3 gene, we report a novel mutation causing an amino acid change (Ala202Val) at the same locus. Our other reported mutation (Cys579Arg) correlates well with known mutations in NOTCH3, as the majority of CADASIL-associated mutations in NOTCH3 generally occur in EGF-like repeat domain, causing changes in the number of cysteine residues.

${ }^{1}$ Seminars in Cerebrovascular Diseases and Stroke 2004; 4:102-108.

${ }^{2}$ Nature 1996; 383:707-710.

${ }^{3}$ The Lancet $1995 ; 346: 934-939$.

${ }^{4}$ Journal of the Neurological Sciences 2006:246, 111-115.

${ }^{5}$ Lancet 1997; 350:1511-1515.

${ }^{6}$ Cephalalgia 2006; 26:158-161.

DE NOVO CHROMOSOMAL TRANSLOCATION RESULTING IN ANTLEY-BIXLER SYNDROME

S. Sandaradura',A. Turner', P. Dalzell ${ }^{2}$, P. Sharma ${ }^{3}$

${ }^{1}$ Department of Medical Genetics, Sydney Children's Hospital, Sydney, Australia

${ }^{2}$ Molecular \& Cytogenetics Unit, SEALS, Sydney, Australia

${ }^{3}$ Department of Cytogenetics, The Children's Hospital at Westmead, Sydney, Australia

Antley-Bixler syndrome is a rare inherited condition characterized by manifestations of cytochrome p450 oxidoreductase deficiency including cortisol deficiency and ambiguous genitalia and a range of other features including craniosynostosis, distinctive facial appearance, skeletal anomalies and renal anomalies. The condition is caused by mutations in the cytochrome P450 oxidoreductase gene (POR; OMIM\#124015) on chromosome 7q11.2. Inheritance is autosomal recessive. We describe a female infant born at full term with typical features of Antley-Bixler syndrome, including an unusual skull shape, frontal bossing, midface hypoplasia, depressed nasal bridge, low set ears, widely spaced nipples, flexion contractures of the elbow, camptodactyly and congenital heart disease (atrial septal defect). Karyotype showed a translocation; $\mathrm{t}(5 ; 7)(\mathrm{q} 15 ; \mathrm{q} 11.2)$. We discuss the molecular basis of Antley-Bixler syndrome in this patient, including results of array CGH in the proband, mutation analysis of the POR gene and parental karyotypes and implications for recurrence risk in this family.

\section{TRPV4 RELATED SKELETAL DYSPLASIAS: A PHENOTYPIC SPECTRUM HIGHLIGHTED BY CLINICAL, RADIOGRAPHIC, AND MOLECULAR STUDIES IN 22 NEW FAMILIES.}

R. Savarirayan', E. Andreucci' ${ }^{2}$ S.Aftimos ${ }^{3}$, M.Alcausin ${ }^{4}$, A. Zankl' ${ }^{12}$, E. Haan $^{5}$,

W. Hunter ${ }^{6}$, P. Kannu', B. Kerr ${ }^{8}$, G. McGillivray', S. Maitz ${ }^{15}$, M. Gardner ${ }^{3}$,

M. Patricelli ${ }^{9}$, D. Sillence ${ }^{4}$, E. Thompson ${ }^{5}$, M. Zacharin ${ }^{11}$, S. Lamande ${ }^{10}$

1 Victorian Clinical Genetics Service, Melbourne, Australia

2 Meyer Childrens Hospital, Florence, Italy

3 Northern Regional Genetic Service, Auckland, New Zealand

${ }^{4}$ Genetic Medicine, University of Sydney, Australia

5 SA Clinical Genetics Services, Adelaide, Australia

${ }^{6}$ Palmerston North Hospital, Palmerston North, New Zealand

${ }^{7}$ Genetics and Metabolic Medicine, Hospital Sick Children, Canada

${ }^{8}$ Genetic Medicine, St Mary's Hospital, Manchester, Australia

${ }^{9}$ Clincical Molcular Biology, Milan, Italy

${ }^{10}$ Murdoch Childrens Research Institute, Australia

${ }^{11}$ Diabetes and Endocrinology, Royal Children's Hospital, Australia

${ }^{12}$ Genetic Health Queensland, Australia

${ }^{13}$ University of Queensland, Australia

${ }^{14}$ SA Clinical Genetics Services, Australia

${ }^{15}$ Clinical Paediatric Genetics, Monza, Italy

Background: The TRPV4 gene encodes a calcium-permeable ionchannel that is widely expressed, responds to many different stimuli and participates in an extraordinarily wide range of physiologic processes. Autosomal dominant brachyolmia, spondylometaphyseal dysplasia Kozlowski type (SMDK) and metatropic dysplasia (MD) are three distinct skeletal dysplasias which share common clinical features, including short stature, platyspondyly, and progressive scol- iosis. Recently, mutations in TRPV4 have been found to be responsible for these three skeletal phenotypes. Methods and Results: We critically analyzed the clinical and radiographic data on 27 subjects from 22 families, all of whom had a clinical diagnosis of one of the conditions described above: 16 with MD; 9 with SMDK; and 2 with brachyolmia. We sequenced TRPV4 and identified 9 different mutations in 23 patients, 4 previously described, and 5 novel. There were 4 mutation-negative cases: one with MD and one with SMDK, both displaying atypical clinical and radiographic features for these diagnoses; and two with brachyolmia, with isolated spine changes and no metaphyseal involvement. Conclusions: Our data confirm autosomal dominant brachyolmia, MD, and SMDK as allelic TRPV4 skeletal dysplasias, and suggest that these conditions represent a continuum of severity with areas of phenotypic overlap, even within the same family. We propose that $\mathrm{AD}$ brachyolmia lies at the mildest end of this spectrum and, since all cases described with this diagnosis and TRPV4 mutations display metaphyseal changes, we suggest that is not a distinct entity but represents the mildest phenotypic expression of SMDK.

\section{MOTIVATIONS, DESCRIPTIVE EPIDEMIOLOGY AND OUTCOMES OF PATIENTS SEEKING GENETIC TESTING FOR HUNTINGTON DISEASE}

T. Scuffham, J. MacMillan

Genetic Health Queensland, Queensland Health, Brisbane, Australia

Introduction: Questions continue to surround genetic testing for HD including the demand for testing, the characteristics of those who seek testing and their motivations, and the proportion who complete the testing process. This information is important for designing and delivering genetic testing for HD. This study aims to describe and quantify the epidemiology, social determinants and outcomes of those seeking genetic testing for HD. Methods/Patients: Medical charts of patients referred between 2006 and 2010 to a large statewide genetics service in Australia (Genetic Health Queensland, GHQ) were audited. Data extracted included demographic characteristics, service features (such as time from referral to completion of testing), test results and motivations for testing. Descriptive analysis was undertaken. This project presents early findings from the sample audited to date. Results: From a sample of 131 patients, the mean age at referral was $39.1(+/-14.4)$ years and $56 \%$ were female. Most patients were referred by GPs (79\%), neurologists (10\%), psychiatrists $(3 \%)$ or other specialists $(8 \%)$ for predictive testing $(78 \%)$, diagnostic testing (18\%) or review (4\%). Patient motivations for testing include future planning $(21 \%)$, diagnosis $(17 \%)$, need to know $(15 \%)$ and family planning $(14 \%)$. The average time to complete testing from initial referral to receiving results was $226(+/-$ 134 ) days and $64 \%$ of predictive tests had $\geq 40$ CAG repeats. Conclusion: Preliminary results indicate there is wide variation in the time to go through the testing process. Further research to identify the determinants and outcomes of those seeking genetic testing for HD will be undertaken.

PTEN MUTATION ANALYSIS RESULTS FROM THE FAMILIAL CANCER LABORATORY OF SA PATHOLOGY

J. C. Seymour', T. Bradford', G. McKavanagh', C. Barnett², G. Suthers ${ }^{3}$, L. Rawlings'

1 Molecular Pathology, SA Pathology, Adelaide, Australia

2 South Australian Clinical Genetics Services, Women's and Children's Hospital, Adelaide, Australia

3 South Australian Familial Cancer Unit, Women's and Children's Hospital, North Adelaide, Australia

In 1997, germline mutations in the tumor suppressor gene PTEN, protein phosphotase with homology to tensin, were found to be associated with a majority of cases of Cowden syndrome (CS). This is an autosomal dominant heritable multiple hamartoma syndrome with an associated high risk of breast, thyroid and endometrial cancer. PTEN mutations have also been linked to other hamartoma disorders such as Bannayan-Riley-Ruvalcaba syndrome (BRRS) and Proteus 
syndrome (PS). The known function of the PTEN protein is to negatively regulate P13K/AKT signaling, thereby reducing the downstream activation of pathways for cell survival and proliferation. Deactivating mutations in the PTEN gene therefore have the potential to lead to multiple tissue overgrowth and cancer. Since May 2009 the Familial Cancer Laboratory routinely performed PTEN full gene screens by DNA sequencing and Multiplex Ligation-dependent Probe amplification (MLPA). To date, the laboratory has performed full gene screens on 35 patients, partial gene screens on 7 patients, and predictive mutation screens for 33 patients who are relatives of positive patients. Confirmatory testing for 2 patients with mutations identified in other laboratories have also been performed. The mutations identified by this laboratory are: c.17_18 del (exon 1), c.217G > T (exon 4), c.304_305del3ins2 (exon 5); c.412T $>\mathrm{G}$ (exon 5), c.424C $>\mathrm{T}$ (exon 5), c.518G $>$ A (exon 6), c.1000A $>\mathrm{T}$ (exon 8), and c.1003C $>\mathrm{T}$ (exon 8). PTEN mutation analysis is requested to obtain to a better diagnosis for the clinical management of patients. As in all genetic cancer disorders, the implications for family members must also be considered. The results of the tests we offer have provided clinicians and genetic counselors with important information to manage both aspects.

\section{CASE STUDY: PARTIAL TRISOMY OF XP22.13 IN A CHILD} WITH DEVELOPMENTAL DELAY

\section{K. L. Sheath', A. Al-Murrani', S. Aftimos ${ }^{2}$ \\ 1 Diagnostic genetics, Auckland District Health Board, Auckland, New Zealand \\ 2 Northern Regional Genetic Services, Auckland District Health Board, Auckland, New Zealand}

Female carriers of balanced translocations between an X chromosome and an autosome can exhibit a range of phenotypes from apparently normal to those with a range of abnormalities. This is due almost entirely to a skewed $\mathrm{X}$ inactivation pattern resulting in functional $\mathrm{X}$ disomy. In phenotypically normal females the translocated $\mathrm{X}$ is preferentially activated and the intact $\mathrm{X}$ is inactivated. From a counseling perspective, this presents problems; there are many possible genotypic outcomes for offspring of a balanced carrier and unlike an autosomeautosome translocation, there is the added complication of estimating risk when there is the possibilty of functional disomy. We present a case of a 17/12 infant with developmental delay and failure to thrive, with a $17 \mathrm{Mb}$ duplication of Xp22.33p22.13 detected by microarray and found to be maternally inherited. We present the results of $\mathrm{X}$ inactivation methylation studies on both mother and daughter and possible outcomes for future pregnancies.

\section{IL28B SNP ANALYSIS IN HEPATITIS C PATIENTS: THE AUCKLAND EXPERIENCE}

\section{N. Singh}

Diagnostic Genetics, LabPlus, ADHB, Auckland, New Zealand

Introduction: Currently $3 \%$ of the global population is infected with the Hepatitis C Virus (HVC) and liver damage caused by chronic $\mathrm{HCV}$ infection is the number one cause of liver transplant in the Western world. Treatment is expensive, has numerous side effects and patients with HCV genotype 1 have only 50\% chance of being cured. Pharmacogenomic studies indicate a single nucleotide polymorphism (SNP) close to the IL28B gene ( rs1 2979860) is a strong predictor of a patient's likelihood of responding to HCV treatment. We report here a test for rs 12979860 that is appropriate for routine diagnostic laboratories. Methods: DNA was extracted from the blood of HCV genotype 1 patients and the rs12979860 SNP status (C/T allele) was determined by T-ARMS-PCR by an adaption of the method of Galmozzi et al. ${ }^{1}$ Confirmation of SNP status and validation of the T-ARMS-PCR protocol was obtained by sequencing analysis. Results: The Galmozzi et al. method as published could not be satisfactorily replicated by our lab. However, sequence alteration of the primer IL28B_ri enabled the $\mathrm{C}$ and $\mathrm{T}$ alleles at this locus to be distinguished. This modified protocol was validated by direct sequencing with $100 \%$ concordance between the two methods. While the reported allele frequency for this locus in European populations is $\mathrm{C}=0.8 / \mathrm{T}=0.2$, the allele frequency detected in HCV genotype 1 patients in Auckland was $\mathrm{C}=0.6 / \mathrm{T}=0.4$. Conclusion: This study has successfully adapted a method for the detection of the rs1 2979860 SNP to an assay that can be easily interpreted and is appropriate for high-throughput diagnostic testing. The lower than expected frequency of the $\mathrm{C}$ allele in $\mathrm{HCV}$ genotype 1 patients in Auckland supports the claim that the $\mathrm{C}$ allele at this locus improves a patients chances of spontaneous viral clearance and recovery from $\mathrm{HCV}$ infection.

${ }^{1}$ Journal of Viral Hepatitis 2011; 18.

EXTENDING THE SCOPE OF DIAGNOSTIC CHROMOSOME ANALYSIS: DETECTION OF SINGLE GENE DEFECTS USING HIGH RESOLUTION MICROARRAYS

H. Slater ${ }^{1,2}$, D. Bruno ${ }^{1,2}$, Z. Stark ${ }^{1,2}$

${ }^{1}$ Victorian Clinical Genetics Services, Melbourne, Australia

${ }^{2}$ Murdoch Childrens Research Institute, Melbourne, Australia

Molecular karyotyping by microarray has provided significant improvement in the diagnosis of disorders resulting from chromosome abnormalities through detection of submicroscopic pathogenic copy number changes. Consequently, a consensus statement has recommended that array based technology should be used as a 'first tier' test in the evaluation of individuals with intellectual disability, developmental delay, multiple congenital anomalies and autism spectrum disorders. The availability of arrays with increasingly high probe coverage and resolution has increased the detection of decreasingly small copy number changes. Most copy number changes represent benign polymorphisms but a small proportion prove to be pathogenic or are novel and of unknown clinical significance. The highest resolution arrays, especially those providing SNP genotyping, provide resolution down to about $5 \mathrm{~kb}$, which permits detection of copy number changes at the single gene or even exon level. Furthermore, these arrays also detect regions with 'loss of heterozygosity'. Of special note are cases showing a single such region containing a recognized gene with a pathogenic homozygous deletion or sequence mutation. We describe 13 examples of pathogenic single gene defects which were found in a consecutive set of 5000 microarray tests using a variety of high resolution SNP microarrays. These cases illustrate the increased scope of cytogenetic testing beyond dominant chromosome rearrangements which typically contain many genes. Seven cases were diagnosed clinically and the array analysis detected a pathogenic copy number change consistent with this, that is, the diagnosis followed a 'phenotype to genotype' approach. Six cases were diagnosed by the laboratory analysis without a specific or differential clinical diagnosis, ie followed a 'genotype to phenotype' approach. Two diagnoses were 'incidental', that is, unrelated to the original reason for testing. These results emphasise the importance of astute clinical diagnosis, especially for such rare genetic disorders and also of providing detailed phenotypes for the laboratory analyst.

\section{DELIVERY OF AN EDUCATION DAY FOR CAREGIVERS OF ADULTS WITH LATE DIAGNOSED PHENYLKETONURIA AS AN ALTERNATIVE APPROACH TO INDIVIDUAL COUNSELING TO ADDRESS PROBLEMATIC AREAS OF PATIENT MANAGEMENT}

\section{A. M. Smith', F. G. Bowling ${ }^{2}$}

1 Nutrition \& Dietetics, Mater Children's Hospital, Brisbane, Australia

2 Metabolic Services, Mater Children's Hospital, Brisbane, Australia

Background: The Metabolic Clinic at the Mater Children's Hospital, Brisbane, manages eight adult patients with late diagnosed phenylketonuria (PKU) living in supported accommodation. Patients attend individual clinic appointments with at least one carer. However, patients are not always accompanied by the same carer(s) making education and management of these patients problematic which can adversely affect patient phenylalanine (Phe) and tyrosine (Tyr) levels. Many patients also have a body mass index (BMI) either above or below the healthy BMI range for adults. Aims: The aim of the new 
workshop was to educate carers of patients with late diagnosed PKU on relevant aspects of the management of PKU. The three key aims were to lower Phe levels within acceptable range, improve the Phe/Tyr ratio and achieve a healthy BMI for patients. Method: All carers of a patient with late diagnosed PKU were invited to attend the workshop co-facilitated by the metabolic physician and dietitian. The session covered topics usually discussed in individual sessions, including dietary management of PKU, importance of blood monitoring and maintaining a healthy body weight. Questions and discussion from the group were encouraged. Results: Eighteen carers (representing five of the eight patients) attended the workshop. Preliminary data indicate patients who had mean Phe levels above target range (400-700 $\mu \mathrm{mol} / \mathrm{L}$ ) prior to the workshop have improved and maintained mean Phe levels for the 11 months post session. Patients who had a BMI above or below the healthy BMI range have improved their BMI . Further data will emerge as evaluation continues. Conclusion: This was the first education workshop specifically designed for carers of patients with late diagnosed PKU in Queensland. We have demonstrated an improvement in Phe levels, Phe/Tyr ratio and BMI for patients who had carers attend the session. Future annual workshops will assess attendees knowledge and measures of social support to further improve session delivery.

\section{THE CC GENOTYPE OF THE RS6505162 C<A SNP IN MIR423 MAY CONFER REDUCED RISK OF BREAST CANCER DEVELOPMENT}

\section{R.A. Smith, D. Jedlinski, P. Gabrovska, L. Haupt, L. R. Griffiths}

Griffith Health Institute, Griffith University, Australia

Background: Micro-RNAs (miRNAs) are small ( 21 nucleotide) single stranded RNA fragments that are involved in regulating of the expression of many genes by inhibiting the translation of mRNAs to which they bind. Micro-RNA 423 (miR423) has previously been implicated in cell differentiation and the development of neuroblastomas and cervical cancer. In addition, an $\mathrm{A}<\mathrm{C}$ single nucleotide polymorphism in the miR423 pre-RNA sequence has previously been indicated to affect the risk of developing cervical cancer in BRCA2 mutation carriers. We undertook this study to examine the effect of the miR423 A<C SNP (rs6505162) on breast cancer risk. Methods: An association population consisting of 179 breast cancer sufferers and their age and sex matched controls underwent PCR for the rs6505162 SNP. Following PCR, genotypes for the population were determined using high resolution melting. Analysis of the data was then performed using the chi-square method. Results: Analysis indicated that there were significant differences in genotype distribution between cancer and control populations, with the $\mathrm{CC}$ genotype being less common in the cancer population $(p=.035)$. Differences in allele frequencies were detected, with the $\mathrm{C}$ allele being rarer in cancer, but these were not significant $(p=.054)$. Discussion: The results of this research indicate that the rs6505162 SNP may be involved in the development of breast cancer. Although our results indicate that allele frequencies are not significantly different between cases and controls, previous research on rs6505162 in oesophageal and cervical cancer has indicated that only the homozygous CC genotype confers significantly altered risk. Our results show similar results to previous research on oesophageal cancer, but a reversed trend from cervical cancer, where the $\mathrm{CC}$ genotype was associated with higher cancer risk. Breast and ovarian cancer risk have previously been found to be inversely correlated, however, so miR423 may participate in pathways that contribute to these differences.

\section{HUMAN VARIOME PROJECT AUSTRALIAN NODE}

\section{T. D. Smith}

Human Variome Project Australian Node, Melbourne, Australia

The Australian Node of the Human Variome Project is an integrated data collection system designed to aggregate genetic test results in a single national repository and link these results with corresponding clinical information. Test results are automatically collected from Australian pathology labs conducting genetic testing and made available via the Australian Node Portal to doctors, counselors and staff of registered genetic testing laboratories within Australia as well as medical research scientists conducting HREC approved projects. Collecting all genetic test results at the national level has several benefits to both medical research and clinical delivery. Pathology labs, whose remit is to test individuals for genetic changes and provide a report on the potential functional consequences of any discovered changes, will be able to draw on the collective experience of the entire country's pathology labs and will provide an additional resource to inform such interpretations. Pathology labs will also be able to use this new information resource to design multi-institution quality assurance programs. By linking the test result data within the Australian Node to clinical data from hospitals and clinics within the country, medical researchers will be able to discover new insights into genetic disorders that may one day lead to better diagnosis and treatment options for affected individuals and their families, as well as creating a rich research platform in the areas of medical science, health care and bioinformatics. The Australian Node of the Human Variome Project is just one of several HVP Country Nodes being actively developed around the world. Other countries include: Kuwait, Egypt, Belgium, China and Malaysia. These repositories, or HVP Country Nodes, are built, managed and resourced by individual countries to meet the information, ethical and legal requirements of those individual countries. The Human Variome Project Consortium facilitates their construction and operation via the creation of standards and systems, examples of which include: nomenclature, data visualization tools and database software.

\section{CURRENT PRACTICE AND ATTITUDES OF AUSTRALIAN OBSTETRICIANS TOWARDS POPULATION-BASED CARRIER SCREENING FOR INHERITED CONDITIONS \\ Z. Stark ${ }^{1,2}$, J. Massie ${ }^{3,4}$, L. loannou ${ }^{2}$, N. Cousens ${ }^{2}$, B. McClaren ${ }^{5}$, S. Lewis ${ }^{6}$, S. Metcalfe ${ }^{4,5}$, M. Delatycki $i^{2,4,7}$ \\ ${ }^{1}$ Clinical Genetics, Genetic Health Services Victoria, Melbourne, Australia ${ }^{2}$ Bruce Lefroy Centre for Genetic Health Research, Murdoch Childrens Research Institute, Melbourne, Australia \\ ${ }^{3}$ Department of Respiratory Medicine, Royal Children's Hospital, Melbourne, Australia ${ }^{4}$ Department of Paediatrics, University of Melbourne, Australia \\ ${ }^{5}$ Genetics Education and Health Research, Murdoch Childrens Research Institute, Melbourne, Australia \\ ${ }^{6}$ Genetic Epidemiology, Murdoch Childrens Research Institute, Melbourne, Australia \\ ${ }^{7}$ Clinical Genetics, Austin Health, Melbourne, Australia}

Introduction: Population-based carrier screening aims to identify asymptomatic carriers for genetic conditions and give prospective parents reproductive options to prevent the birth of affected children. Several programs targeting specific conditions are in existence worldwide and have demonstrated dramatic decreases in the incidence of affected infants with the conditions being tested for. The frequency with which obstetricians and other pregnancy health care providers offer tests to patients is likely to be a major determinant of the uptake of population-based carrier screening programs. Methods: Australian Fellows of the Royal Australian and New Zealand College of Obstetricians and Gynaecologists (RANZCOG) were invited by email to complete a voluntary anonymous online questionnaire regarding their current practice and attitudes towards population-based carrier screening. Results: 237/1121 (21.1\%) Fellows responded to the questionnaire. Of these, $83 \%$ expressed in principle support for population-based carrier screening for at least some conditions, with $97 \%$ supporting carrier screening for $\beta$-thalassemia, and $83 \%$ supporting carrier screening for cystic fibrosis (CF). Within this broad support, opinions ranged from enthusiastic endorsement to total opposition, and even accusations of 'eugenics'. A very small number of obstetricians reported offering carrier screening as part of routine pregnancy care $(20 \%$ for $\beta$-thalassemia, $8 \%$ for $\mathrm{CF}, 5 \%$ for fragile $\mathrm{X}$ syndrome, $2 \%$ for spinal muscular atrophy). The main practical barriers to screening identified were 
cost, time constraints, and availability of supporting services. Only a minority of respondents $(6 \%)$ felt that such screening is best offered as part of routine pregnancy care, with most favoring pre-conceptual screening in adulthood. Conclusions: There is broad support among responding obstetricians for population-based carrier screening for inherited conditions, although translation into routine practice is limited by a number of practical barriers and wider concerns. Addressing these issues is a crucial element for the successful implementation of any population carrier screening program.

\section{MÜLLERIAN ANOMALIES SHOULD BE CONSIDERED A COMPONENT OF THE CLINICAL PHENOTYPE OF 16P11.2 DELETION SYNDROME.}

R. D. Susman', R. M.N. Kimble ${ }^{2}$

${ }^{1}$ Genetic Health Queensland, Royal Brisbane and Women's Hospital, Brisbane, Australia

${ }^{2}$ Obstetrics and Gynaecology, Royal Brisbane and Women's Hospital, Brisbane, Australia

With the increasing use of array comparative genomic hybridization (aCGH), 16p11.2 deletion syndrome is becoming increasingly recognized. The recurrent $\sim 550 \mathrm{~kb}$ deletion (from $29.5-30.1 \mathrm{Mb}$ ) is associated with variable clinical phenotypes including delayed language development, intellectual disability, autism, and congenital anomalies. Some people with this deletion have a normal phenotype. We report a 16-year-old girl with multiple problems including intellectual disability, speech and language impairment, epilepsy, and Müllerian anomalies who has a 16p11.2 deletion. We will present further details of her phenotype and review the literature.

\begin{tabular}{l}
\hline UNDERSTANDING WOMEN'S DECISIONS ABOUT \\
PRENATAL DIAGNOSIS FOR CHROMOSOME \\
ABNORMALITIES.
\end{tabular}

M. Susman', J. L. Halliday', D.Amor ${ }^{2}$, J. Bayer ${ }^{3}$

${ }^{1}$ Public Health Genetics, Murdoch Childrens Research Institute, Melbourne, Australia

${ }^{2}$ Genetic Health Services Victoria, Melbourne, Australia

${ }^{3}$ Centre for Community Child Health, Murdoch Childrens Research Institute, Melbourne, Australia

Background: The introduction of targeted non-invasive diagnosis may result in pregnant women being able to choose which chromosome conditions they want detected, yet little is known about their opinions on prenatal detection of chromosome abnormalities with varying severity, particularly those less severe and less understood than Down syndrome. Objective: To determine if women differentiate between prognostic outcomes of different chromosome conditions when deciding to have a hypothetical non-invasive diagnostic test at 12 weeks gestation. Method: Women's beliefs elicited from a preliminary qualitative questionnaire were used to construct the main questionnaire. The target population comprised pregnant women, less than 20 weeks gestation, who had a screening test for Down syndrome. Four different chromosome conditions (Down syndrome, Klinefelter syndrome, triple $\mathrm{X}$ syndrome, and mosaic trisomy 20 at amniocentesis) were included, each questionnaire being about one condition. A short description of each condition was provided, labeled condition A to D. The questionnaire was designed to measure four variables; intention to take the test, attitude, perceived control, and social norm influences. Results:

\begin{tabular}{lrrrr} 
Intention to test & \multicolumn{2}{l}{ Down syndrome $(N=52)$} \\
$\%$ & \multicolumn{4}{l}{ Klinefelter syndrome $(N=51)$} \\
$\%$ & \multicolumn{4}{l}{ Triple X syndrome $(N=41)$} \\
$\%$ & \multicolumn{4}{c}{ Mosaic trisomy 20 at amniocen } \\
$\%$ & & & & \\
Yes & 97.2 & 76.5 & 74.5 & 80.6 \\
No & 1.0 & 16.7 & 7.4 & 4.0 \\
Unsure & 1.9 & 6.9 & 18.3 & 15.3
\end{tabular}

Other results to be presented include (1) predicting intention from attitude, perceived control, and social norm, and (2) predicting intenders versus non-intenders from specific beliefs. Conclusion: Most pregnant women currently having screening for Down syndrome want testing for less severe conditions (e.g., Klinefelter and triple X syndromes) and for conditions where there is considerable prognostic uncertainty (e.g., mosaic trisomy 20). There is, however, an important but small minority, who are unsure or would not want testing for these conditions.

\section{IDENTIFYING EPIGENETIC VARIANTS THAT INFLUENCE CARDIOVASCULAR RISK IN THE NORFOLK ISLAND POPULATION ISOLATE}

H. G. Sutherland', R.A. Lea', J. Blangero ${ }^{2}$, L. R. Griffiths'

1 Griffith Health Institute, Griffith University, Southport, Australia

2 Department of Genetics, Southwest Foundation for Biomedical Research, San Antonio, Texas, United States

Cardiovascular disease (CVD) is of major importance to public health as it accounts for approximately 50\% of deaths in Western societies. Risk factors for CVD show strong familial tendencies but involve both environmental and genetic components, although the genetics of CVD risk remains unclear. This study will investigate the genetic basis of CVD risk factors in a well-characterized, homogeneous population from Norfolk Island. The Norfolk Island community is a unique population isolate derived primarily from 18th century English Bounty mutineers and Polynesian women who relocated to Norfolk Island from Pitcairn in the 1850s. Known identifiable risk factors such as blood pressure, body weight, lipid levels and smoking status, have already been characterized from a significant proportion of this population and DNA from blood has been obtained for genetic studies. A full genome linkage and association scan and an expression QTL (eQTL) scan is currently underway for the Norfolk Island cohort using DNA prepared from these samples. I will then carry out a genome-wide DNA methylation scan in the Norfolk Island population isolate to investigate epigenetic variants that correlate with the CVD risk traits of hypercholesterolemia, hypertension and obesity. This will be done by interrogating Illumina Human 450K Infinium Methylation Beadchips with bisulfite-treated DNA prepared from peripheral blood mononucleocytes. Furthermore, for 318 individuals, phenotypic information and genetic samples were collected in 2000, and again in 2010, so I can also determine how methylation patterns change with time. Epigenetic information will then be incorporated with the other genomic data for the Norfolk Island cohort, providing a unique opportunity to undertake an integrative genomic profiling approach to identify the various interacting components that influence CVD risk traits. Subsequently I will validate loci of interest in independent cohorts of patients measured for the same CVD risk traits.

HETEROZYGOUS AND HOMOZYGOUS 130 DELETION IN CHRONIC LYMPHOCYTIC LEUKEMIA

P. Sutton-Davies, R. Brookwell, H.Wordsworth

Cytogenetics, Sullivan Nicolaides Pathology, Brisbane, Australia

Chronic lymphocytic leukemia is the most frequently occurring leukemia in many parts of the world. The abnormal cells are difficult to encourage to divide in culture, so FISH analysis is often the method of choice to detect clinically significant genetic changes. The most frequent changes are $13 \mathrm{q} 14$ deletions, $11 \mathrm{q}$ deletions, Trisomy 12, 17p deletions, 6q deletions and 14q32 rearrangements. These may have prognostic implications, for example $11 q$ and $17 p$ deletions are signs of poor prognosis. 13q deletion is thought to be a positive prognostic indicator, but recent studies have indicated that this may differ, depending on the abnormal cell load at presentation, and possibly on whether the deletion is heterozygous or homozygous. The latter is controversial, as evidence has been presented for and against. We studied a cohort of 250 patients with $13 \mathrm{q}$ deletion, in order to establish whether homozygous deletions were associated with more advanced disease, and the results suggest that there is such a relationship. 


\section{FISH CONFIRMATION OF MICROARRAY RESULTS: A VALUABLE INSTRUMENT IN INTERPRETATION AND REPORTING}

S. M. Suwalski, J. Nicholl,W.Waters, S.Yu

Cytogenetics, SA Pathology, Adelaide, Australia

FISH confirmation is used to confirm all copy number variations (CNVs) identified by oligo microarray in this laboratory, whether considered to be of known, unknown or unclear clinical significance. We however exclude deletions less than 200kb and duplications less than $400 \mathrm{~kb}$ with no known intellectual disability/mental retardation causing genes. We request parental FISH studies to assist in the interpretation of the array findings before reporting, unless the CNV clearly fits the clinical phenotype (often severe). FISH probes are generated by positioning the change using the Ensembl browser linked to Bluegnome software, choosing an appropriate probe from the $32 \mathrm{~K}$ probe set and picking the DNA from the library stock. DNA is amplified using a Qiagen Repli-G kit, and are fluorescently labeled using a Vysis Nick Translation kit. Specific cases from the previous 12 months of microarray testing elucidated the importance of FISH follow-up:

- deletions associated with two separate complex translocation cases, $\mathrm{t}(4: 14)$ and $\mathrm{t}(6: 14)$

- two insertional translocation cases, $t(7 ; 21)$ and a $t(X ; 1)$

- two deletions associated with a mosaic apparently balanced translocation, $\mathrm{t}(2 ; 10)$

- a large cryptic duplication of chromosome 20p associated with a translocation, $\mathrm{t}(20 \mathrm{p} ; 22 \mathrm{p})$

- a PAX 3 gene disruption from a denovo 2;6 translocation in a patient diagnosed with Waardenburg syndrome and with a $2.5 \mathrm{Mb}$ distal deletion also on chromosome 2 , and

- four unbalanced subtelomeric translocation cases, $\mathrm{t}(7 ; 10), \mathrm{t}(8 ; 22)$, $\mathrm{t}(14 ; 17)$, and $\mathrm{t}(\mathrm{X} ; 18)$.

FISH for these cases has demonstrated the importance of identifying the position of CNVs and highlighted the issue of the possible risk to other family members. This information in conjunction with the patient's clinical information also serves as a useful tool in interpreting the microarray findings and can provide helpful detailed information to the clinical picture of the patient for the referring clinician.

\section{THE DILEMMA OF MANAGING NON-PKU HYPERPHENYLALANEMIA}

\section{A. Sweeney', D. Ketteridge ${ }^{2}$, D. Bratkovic ${ }^{2}$}

${ }^{1}$ Nutrition Department, Children, Youth and Women's Health Service, Adelaide, Australia

${ }^{2}$ Metabolic Unit, SA Pathology (WCH), Adelaide, Australia

There has been much discussion about the management of children with phenylalanine levels between $120-600 \mu \mathrm{mol} / \mathrm{l}$ with differing views and lack of evidence regarding the phe level at which dietary intervention should be commenced. From literature and email correspondence, treatment in other countries is commenced at levels varying between above $360 \mu \mathrm{mol} / 1$ to above $600 \mu \mathrm{mol} / \mathrm{l}$. It is not clear the number of raised levels needed before treatment is commenced. In Australia there is a general consensus that dietary treatment would be considered when phe is greater than $400 \mu \mathrm{mol} / \mathrm{l}$. Case report: Male term infant, breastfed, day 3 phe $=99 \mu \mathrm{mol} / \mathrm{l}$, tyr $=$ $51 \mu \mathrm{mol} / \mathrm{l}$; day $10 \mathrm{phe}=361$, tyr $=91$; day $19 \mathrm{phe}=431$, tyr $=91$; day 20 eight phe $=388$, tyr $=113$. Between $1-3$ months weekly phe levels mean $=271 \mu \mathrm{mol} / \mathrm{l}(\mathrm{R} 251-352)$. Between 3-12 months monthly phe levels mean $=283 \mu \mathrm{mol} / \mathrm{l}(\mathrm{R}$ 149-422). Solid foods introduced at 6 months. However, at 1 year phe $=545$, tyr $=123$, parents repeated 2 weeks later and phe $=524$, tyr $=140$. Dietary protein intake assessed as $3.5-4 \mathrm{~g}$ protein $/ \mathrm{kg}$ plus breastfeeds. Between 12-16 months and with a modest reduction in dietary protein, fortnightly phe levels mean $=404 \mu \mathrm{mol} / \mathrm{l}(\mathrm{R}$ 117-600), higher levels were at times of illness. At 16-month review normal development, $2.5 \mathrm{~g}$ protein $/ \mathrm{kg} / \mathrm{day}$ plus one breastfeed, phe $=333$, tyr $=78$. Fortnightly blood tests and regular review continues. The ongoing management of this case raises a number of issues for discussion including the phe level for treatment, early vs. late introduction of protein supplement and associated issues, management of illness, frequency of blood tests and parental anxiety. The use of BH4 in Australia will help define the management of these children, but until BH4 is widely available management plans need to address the above issues.

AN 8-YEAR-OLD BOY WITH ACUTE ENCEPHALOPATHY WHILE ON TREATMENT FOR ACUTE LYMPHOBLASTIC LEUKEMIA (ALL)

R. Swenson I,W. Chan ${ }^{2}$, C. Fraser ${ }^{3}$,A. Paton ${ }^{4}$, D. Coman', A. Inwood 2 , S. Briscoe', K. Hore ${ }^{5}$, J. McGill ${ }^{2}$

${ }^{1}$ Chemical Pathology, Pathology Queensland, Brisbane, Australia

${ }^{2}$ Metabolic Medicine, Royal Brisbane and Women's Hospital, Brisbane, Australia

${ }^{3}$ Oncology, Royal Brisbane and Women's Hospital, Brisbane, Australia

${ }^{4}$ Pharmacy, Royal Brisbane and Women's Hospital, Brisbane, Australia

${ }^{5}$ Queensland Drug Information Centre, Royal Brisbane and Women's Hospital, Brisbane, Australia

Patient History: An 8-year-old boy with previous history of autistic spectrum disorder was diagnosed with acute lymphoblastic leukemia (ALL). While on the intensification phase of chemotherapy for ALL, the patient developed acute encephalopathy with symptoms of drowsiness, slurred speech, lethargy and fluctuating conscious levels. The patient was being treated with 16 different medications including asparaginase, glucocorticoids, co-trimoxazole (trimethoprim and sulfamethoxazole) and methotrexate. Results: Results were as follows:

\begin{tabular}{lllll} 
Sample & Analyte & \multicolumn{2}{l}{ Result } & Reference interval Units \\
Plasma & Ammonium & 180 & $<50$ & $\mu \mathrm{mol} / \mathrm{L}$ \\
& Phenylalanine & 800 & $40-120$ & $\mu \mathrm{mol} / \mathrm{L}$ \\
CSF & Phenylalanine & 54 & $2-15$ & $\mu \mathrm{mol} / \mathrm{L}$ \\
& Homovanillate & 8 & $220-850$ & $\mathrm{nmol} / \mathrm{L}$ \\
& 5-HIAA & 108 & $65-340$ & $\mathrm{nmol} / \mathrm{L}$ \\
Urine & Total Biopterin & 6.3 & $0.5-3.0$ & $\mathrm{mmol} / \mathrm{mol}$ creat \\
& Total Neopterin & 3.4 & $1.1-4.0$ & $\mathrm{mmol} / \mathrm{mol}$ creat \\
& Primapterin & $<0.1$ & $<0.4$ & $\mathrm{mmol} / \mathrm{mol}$ creat
\end{tabular}

Following treatment with Sapropterin (tetrahydrobiopterin), phenylalanine levels decreased from $650 \mu \mathrm{mol} / \mathrm{L}$ to $42 \mu \mathrm{mol} / \mathrm{L}$ ( 4 hours post treatment) and his conscious level improved. Discussion: Glucocorticoids have been shown to inhibit GTP cyclohydrolase, the first step in the synthesis of tetrahydrobiopterin. Trimethoprim has been shown to be a dihydrofolate reductase inhibitor, thus preventing the production of tetrahydrobiopterin from 7,8-dihydrobiopterin. Methotrexate is a dihydrofolate reductase and dihydrobiopterin reductase inhibitor, thus preventing the production of tetrahydrobiopterin from both 7,8-dihydrobiopterin and q-dihydrobiopterin. The patient had been taking methotrexate, trimethoprim and glucocorticoids during the treatment of ALL. The encephalopathic episode corresponded to an increased methotrexate dose, both intrathecally and intravenously, during intensification therapy which decreased the levels of tetrahydrobiopterin. Tetrahydrobiopterin is a co-factor required to produce HVA and 5HIAA from phenylalanine and tryptophan respectively. The decrease in tetrahydrobiopterin caused an increase in phenylalanine and biopterin. The pathway to the production of HVA has two tetrahydrobiopterin dependent enzymes whereas for the production of 5-HIAA there is only one tetrahydrobiopterin dependent enzyme, so this may explain why the HVA was decreased while the 5-HIAA was normal. 


\section{FABRY DISEASE IN NSW}

M. C.Tchan', K. Devine', C.J. Ellaway' ${ }^{2}$, D. O. Sillence'

${ }^{I}$ Genetic Medicine, Westmead Hospital, Sydney, Australia

${ }^{2}$ Biochemical Genetics, Childrens Hospital at Westmead, Sydney, Australia

A review of the NSW Lysosomal Storage Disorders Management Clinic documented 54 patients with Fabry disease. This included 29 women and 25 men, among whom there were 6 children. There were 25 different mutations in the GLA gene documented amongst this cohort; 2 patients had not had genetic sequencing to determine their mutation. 19 patients had documented cerebrovascular disease with a history of TIA/CVA or white matter changes on brain MRI. 13 patients had stage 2 chronic kidney disease (CKD), 1 patient had stage $3 \mathrm{CKD}, 2$ patients had undergone renal transplantation and 2 patients were on dialysis. 5 patients had a history of atrial fibrillation and 21 patients had documented left ventricular hypertrophy. Self assessments of quality of life (via SF-36 questionnaire) demonstrated a significant decrease in physical component scores compared to the general Australian population (mean PCS $=43$ c.f. 49.7). 18 patients had commenced enzyme replacement therapy, 16 of whom were male. The average duration of therapy was 4.4 years.

\section{A 7-YEAR-OLD BOY WITH MOSAICISM FOR A SMALL SUPERNUMERARY, XIST-NEGATIVE, RING X CHROMOSOME}

E. M.Thompson', J. Samuel ${ }^{2}$, F.Webb ${ }^{2}$,W.Waters ${ }^{2}$, J. Nicholl ${ }^{2}$

${ }^{\prime}$ SA Clinical Genetics Service, SA Pathology, Women's and Children's Hospital, Adelaide, Australia

${ }^{2}$ Department of Cytogenetics, SA Pathology, Women's and Children's Hospital, Adelaide, Australia

A boy with mosaicism for an extra ring chromosome derived from the $\mathrm{X}$ chromosome is described. The ring does not contain the $\mathrm{X}$ inactivation gene, XIST. The ring has occurred de novo, as parental lymphocyte karyotypes show no abnormalities. The boy, now aged 7 years, has borderline/low average intellectual functioning, behavioral problems with attention deficit-hyperactivity disorder, dysmorphic facial features, a well-formed accessory nipple and a prematurely fused metopic cranial suture which required surgical release. Supernumerary tiny ring $\mathrm{X}$ chromosomes are rare but are more common in females. There appear to be very few male cases reported. A chromosome microarray is being done to assess gene involvement.

\section{SIMPLIFYING THE KETOGENIC DIET - EXPERIENCE IN PYRUVATE DEHYDROGENASE COMPLEX DEFICIENCY}

S. M.Thompson ',2,T. Dalkeith', K. Bhattacharya ${ }^{1,3}$, C. Ellaway ${ }^{1,3}$, J. Christodoulou ${ }^{1,3}$ ${ }^{1}$ Western Sydney Genetics Program, The Children's Hospital at Westmead, Sydney, Australia

${ }^{2}$ Department of Nutrition and Dietetics, The Children's Hospital at Westmead, Sydney, Australia

${ }^{3}$ Disciplines of Paediatrics and Child Health and Genetic Medicine, University of Sydney, Australia

Background: Pyruvate dehydrogenase complex deficiency (PDHCD) comprises a range of phenotypes and may be treated with ketogenic diet. This report details management of four patients on a modified ketogenic diet similar to the modified Atkins diet. In all families there were barriers of language, geographical distance and/or social circumstances to hospital admission and/or complex daily calculations, required by the traditional ketogenic diet. Case Reports: Three patients (3-5 years) commenced the diet at home with some or all of their usual milk drinks substituted with Ketocal®. Patient 1 with non-English speaking parents was then hospitalized to assess the changes needed to food intake, review his feeding ability and to allow intensive dietary instruction. Two families made gradual changes at home to their child's usual eating plan whilst testing urinary ketones twice daily. All were advised on management strategies should urinary ketones exceed $16 \mathrm{mmol} / \mathrm{l}$. Three patients are maintaining urinary ketones in the $1.5-8 \mathrm{mmol} / \mathrm{l}$ range and report clinical improvement (less irritability, improved motor function, less hospital admissions). Patient 1 has had no admissions in the two years since the diet was commenced compared to 4 life threatening metabolic decompensations in the previous 2 years. Another patient has maintained plasma lactates in normal range and the parents report two instances of ataxia and listlessness when urinary ketones were low, resolving with oral fat supplementation. A 17year-old previously on a high-fat diet commenced carbohydrate restriction ( $80 \%$ energy from fat) due to worsening lower limb pain. The diet is well tolerated with some reported clinical improvement. Discussion: A simplified diet to induce ketosis, commenced at home, may be less of a burden in PDHCD, compared to traditional ketogenic diets.

\section{NUTRITIONAL ASSESSMENT OF PATIENTS WITH}

\section{METABOLIC DISORDERS}

S.Thompson 1,2, Q.Yip ${ }^{4}$, B. Dennison ${ }^{2}$, P.Watson ${ }^{2}$, J. Coakley ${ }^{3}$, I.Alexander ${ }^{1,5}$,

K. Bhattach ${ }^{1,5}$, C. Ellaway, ${ }^{1,5,}$ J. Christodoulou ${ }^{1,5}$

${ }^{1}$ Western Sydney Genetics Program, The Children's Hospital at Westmead, Sydney, Australia

${ }^{2}$ Department of Nutrition and Dietetics, The Children's Hospital at Westmead, Sydney, Australia

${ }^{3}$ Department of Clinical Biochemistry, The Children's Hospital at Westmead, Sydney, Australia

${ }^{4}$ School of Health Sciences, University of Newcastle, Australia

${ }^{5}$ Disciplines of Paediatrics and Child Health and Genetic Medicine, University of Sydney, Australia

Background: Restricted diets for metabolic disorders frequently require supplementation of micronutrients to maintain appropriate intakes, with potential for inadequate, excessive and unbalanced intake. Biochemical markers may more accurately assess nutritional adequacy as an adjunct to dietary assessment and a protocol to assess nutritional status for 15 micronutrients for metabolic patients has been implemented at the Children's Hospital at Westmead since 2009. Methods: A chart review of 67 patients, fully or partially tested per protocol, compared blood results to normal ranges. Fortysix $(69 \%)$ were on protein-restricted diets, of which 35 (52\% total) were supplemented with amino acid medical food. Ten (15\%) were on unrestricted diets and $11(16 \%)$ on fat-modified diets. Results: The most common deficiency across diet groups was docosahexaenoic acid (DHA; $76 \%$ of 58 tested). Two patients on protein restricted diets had low plasma vitamin B 12; one was prescribed a vitamin fortified amino acid medical food and the other a vitamin supplement. Low plasma levels of magnesium, zinc and vitamins D and $\mathrm{E}$ were more common when a medical food was not prescribed. Plasma folate was elevated in $93 \%$ patients on medical foods; elevated levels of vitamin $\mathrm{A}, \mathrm{E}$ and riboflavin were also reported. Low levels of plasma manganese were found across all diet groups. Vitamin D status was of concern in unrestricted diets. Discussion: Both elevated and low levels of vitamins, minerals and long chain fatty acids were found in metabolic patients, despite regular assessment of nutrient intake and advice on suitable supplements. Regular nutritional monitoring needs to be an integral part of management of all metabolic patients but simplification of the protocol is required due to parental opposition to the blood volume required. A targeted approach to the most vulnerable nutrients is appropriate, but compliance with or adequacy of supplement recommendations should not be assumed.

ADJUSTING TO THE IMPLEMENTATION OF THE GENETIX GSL-120 AND THE EVALUATION OF THE AUTOMATED CAPTURING TECHNOLOGY IN THE CYTOGENETICS LABORATORY

V.Toh, H. B. Ma, S. P. Chua, L. Gole

Cytogenetics Laboratory, Department of Laboratory Medicine, NUHS, Singapore

With an increasing workload and the need to offer more services to our patients, our laboratory has advanced from using a labor intensive manual capturing process to using an automated scan- 
ning and capturing process. A period of 2 weeks was taken to setup and optimize the GSL-120 as staff gave feedback on the differences between manual analysis under the microscope and the automated scanning and capturing technology. Barcoding was customized to our own labeling formats, which in overall helped to reduce possible human errors such as typo errors, assigning slides to the wrong case, etc. Turnaround times were maintained despite the reduction of 1 headcount, and staff could be reallocated to evaluate and start up FISH services. Failure and suboptimal rates were similar to that before the use of the GSL120 , with staff commenting that it was less strenuous to use the GSL-120 to pick up metaphases on top of having a similar detection rate. The images captured for cancer specimens and peripheral blood samples for constitutional studies resulted in improved analysis with ease. Performing interphase FISH using automatic probe capture was helpful in cases where there was no obstructing background, and this allowed the staff to make swift analysis. Information such as counts could be recorded on the electronic images and analysis could be kept for second opinion and documentation purposes. We concluded that the GSL-120 increases efficiency while not compromising on quality, as staff was still able to maintain the abnormality detection rate and turnaround times even with a reduction in headcount and added workload. The less tangible benefits were reducing fatigue, eye strain and ergonomic stress experienced by staff by reducing the long hours of analysis under the microscope.

\section{NEW APPROACHES TO DIAGNOSIS OF MITOCHONDRIAL DISORDERS IN FIBROBLASTS}

S. Tregoning ${ }^{1,2}$, A. Compton ', A. Frazier ${ }^{1}$, S. Lim ${ }^{1,3}$,W. Salter ${ }^{1,2}$, D. Thorburn ${ }^{1,2,3}$

${ }^{1}$ Murdoch Childrens Research Institute, Melbourne, Australia

${ }^{2}$ VCGS Pathology, Melbourne, Australia

${ }^{3}$ Department of Paediatrics, University of Melbourne, Australia

Skin fibroblasts are not routinely used in enzyme diagnosis of patients with suspected disorders of mitochondrial oxidative phosphorylation (OXPHOS) due to several limitations. First, about half the patients with OXPHOS disorders have tissue-specific enzyme defects that are expressed in skeletal muscle or liver but not fibroblasts. Second, assaying OXPHOS enzymes in fibroblasts by traditional spectrophotometric assays requires the preparation of purified mitochondria from a large number of cells (4 x $175 \mathrm{~cm} 2$ flasks), making it an expensive and time consuming diagnostic test and delaying results for up to several months. We aimed to investigate whether two new immunochemical approaches are able to provide a quicker and cheaper option with comparable sensitivity. Vertical flow immunocapture ('dipstick') assays are available for assessing the activities of OXPHOS complexes I and IV and for pyruvate dehydrogenase complex (PDHC) activity; we currently test the latter by a radiometric assay. We have now performed dipstick assays on cell lines with a range of these enzyme defects and detected deficiencies in the three enzymes in patients with various genetic defects. However, a caveat of the dipstick assays is that patients with mutations in some specific genes show activities in the normal range. We also obtained promising results using immunoblotting with an antibody cocktail to quantify the relative amount of subunits from each OXPHOS complex. This assay fails to detect some (catalytic) enzyme defects but is more sensitive than the traditional spectrophotometric assays for detecting other defects such as mtDNA translation defects. Our preliminary data suggest that using both the dipstick and western blotting approaches together may allow a similar overall sensitivity for detecting fibroblast defects of OXPHOS and perhaps PDHC, using a total volume of cells about one tenth that needed for traditional assays, allowing savings in cost and turn-around time.
MUTATIONS IN MTFMT UNDERLIE A HUMAN DISORDER OF FORMYLATION CAUSING IMPAIRED MITOCHONDRIAL TRANSLATION

E. J.Tucker ${ }^{1,2}$, S. G. Hershman ${ }^{3,4,5}$, C. Köhrer 6 , C.A. Belcher-Timme ${ }^{3,4,5}$, O.A. Goldberger, 3,5, J. Christodoulou ${ }^{7,8,9}$, J. M. Silberstein ${ }^{10}$, M. McKenzie $^{11}$, M.T. Ryan ${ }^{11,12}$,A. G. Compton', S. E. Calvo ${ }^{3,4,5}$, U. L. RajBhandary',

D. R. Thorburn $1,2,13$,V. K. Mootha ${ }^{3,4,5}$

${ }^{1}$ Mitochondrial Research, Murdoch Childrens Research Institute, Melbourne, Australia ${ }^{2}$ Department of Paediatrics, University of Melbourne, Australia

${ }^{3}$ Center for Human Genetic Research, Massachusetts General Hospital, Boston, United States

3 Department of Systems Biology, Harvard Medical School, United States

4 Broad Institute, Cambridge, United States

${ }_{5}^{5}$ Department of Biology, Massachusetts Institute of Technology, United States

${ }^{6}$ Genetic Metabolic Disorders Research Unit, Children's Hospital at Westmead, Sydney, Australia

Discipline of Paediatrics \& Child Health, University of Sydney, Australia

9 Discipline of Genetic Medicine, University of Sydney, Australia

${ }^{10}$ Department of Neurology, Princess Margaret Hospital for Children, Perth, Australia

${ }^{11}$ Department of Biochemistry, La Trobe University, Australia

${ }^{12}$ ARC Centre of Excellence for Coherent X-ray Science, La Trobe University, Australia

${ }^{13}$ Genetic Health Services Victoria, Royal Children's Hospital, Melbourne, Australia

Disorders of oxidative phosphorylation (OXPHOS) affect $\sim 1 / 5000$ live births and are the most common inborn errors of metabolism. OXPHOS requires five multimeric complexes, four of which contain subunits encoded by the mtDNA. Combined OXPHOS deficiency is one of the most common OXPHOS disorders and can potentially result from mutations in $24 \mathrm{mtDNA}$-encoded tRNA or rRNA genes or in at least 26 known nuclear 'disease genes' encoding proteins involved in mtDNA replication, maintenance or translation. The molecular diagnosis of patients with combined OXPHOS deficiency is challenging due to the allelic heterogeneity and the fact that more 'disease genes' likely await discovery. Recent advances in sequencing technology enable the simultaneous investigation of multiple candidate genes via Next-Generation sequencing. Here, we performed targeted Next-Generation sequencing of the mtDNA and the exons of 1381 nuclear genes encoding the mitochondrial proteome (MitoExome) in two unrelated children presenting with Leigh syndrome and combined OXPHOS deficiency. This identified $\sim 700$ single nucleotide variants or small insertion/deletions in each patient. We prioritized rare variants that were likely to have a deleterious impact on protein function and focused on genes that fit a recessive mode of inheritance, which left only one candidate gene in each patient: MTFMT, encoding the mitochondrial methionyl-tRNA formyltransferase. MTFMT catalyzes the formylation of mitochondrial Met-tRNAMet to generate fMet-tRNAMet, which is then used for translation initiation. The absolute requirement for MTFMT has remained in question because disruption of the FMT gene in yeast causes no discernible defect in mitochondrial protein synthesis or function. Fibroblasts from these patients exhibited dramatically reduced fMet-tRNAMet and severe defects in mitochondrial translation that could be rescued by exogenous expression of MTFMT. Our findings demonstrate that MTFMT is a new gene underpinning combined OXPHOS deficiency and that MTFMT is essential for efficient human mitochondrial translation.

REPORTING GENOMIC RESULTS TO PATIENTS: A REVIEW OF AVAILABLE GUIDELINES AND LITERATURE

E. Turbitt ${ }^{1,2}$, D.Amor ${ }^{3,4}$, J. Halliday ${ }^{2,5}$, S. Metcalfe ${ }^{1,2}$

${ }^{1}$ Genetics Education and Health Research, Murdoch Childrens Research Institute, Melbourne, Australia

${ }^{2}$ Paediatrics, University of Melbourne, Australia

${ }^{3}$ Genetic Health Services Victoria, Victorian Clinical Genetics Service, Melbourne, Australia

${ }^{4}$ Clinical Genetics Research, Murdoch Childrens Research Institute, Melbourne, Australia

${ }^{5}$ Public Health Genetics, Murdoch Childrens Research Institute, Melbourne, Australia

Guidelines on the use of microarray technology in Australasian diagnostic laboratories were made available in March 2011. ${ }^{1}$ This 
document, and others like it internationally, have been compiled in response to the rapid integration of DNA microarrays in clinical settings. It has been predicted that further technological developments, including whole genome sequencing, will play an increasing role in improving healthcare. Whole genome testing can uncover genetic changes of uncertain clinical significance, or genetic changes known to have pathological consequences unrelated to the disorder being tested. This potentially raises ethical challenges for laboratory and clinical staff. A literature review of published guidelines, policies and recommendations to determine what guidance scientists, clinicians and patients are given when handling results obtained from genomewide testing is being conducted. Initial findings from the 21 documents reviewed to date indicate that limited or no formal policies exist at a national or international level regarding consent and reporting information from genome-wide testing to patients. It has been shown that discrepancies exist in interpreting and reporting results produced from microarray technologies. ${ }^{2}$ Therefore, although internal policies may be in place, it can be predicted that variations exist in the content of these policies and how they are applied. A limited number of studies have investigated the effects on patients receiving genomic results. Furthermore, little is known about how much information patients actually want to know. We have designed a qualitative study to investigate the current protocols and resulting impacts surrounding the use of new genomic testing technologies in routine clinical care. This research will provide information about how these impacts can be managed to minimise adverse consequences, and data gathered will contribute to the development of optimal guidelines for reporting genomic information to patients.

${ }^{1}$ Australasian Society of Cytogenetics, 2011, Best Practice Microarray Guidelines for Australasian Laboratories, Human Genetics Society of Australasia.

${ }^{2}$ Genetics in Medicine, 2009; 11:866-873.

\section{A SPECTRUM OF NEONATAL HYPOPHOSPHATASIA: TWO CASE REPORTS.}

\author{
M.A.Van Werkhoven', C. Theda ${ }^{2}$,J.J. Pitt ${ }^{1,3}$ \\ ${ }^{1}$ VCGS Pathology, Murdoch Childrens Research Institute, Melbourne, Australia \\ ${ }^{2}$ Royal Women's Hospital, Melbourne, Australia \\ ${ }^{3}$ Department of Paediatrics, University of Melbourne, Australia
}

Hypophosphatasia is a metabolic bone disease characterized by defective skeletal mineralization caused by low activity of tissuenonspecific isoenzyme of alkaline phosphatase (TNSALP). Here we describe two patients with a spectrum of neonatal hypophosphatasia. Patient A has a severe form in which prenatal ultrasounds established the presence of a severe skeletal dysplasia as all limbs were shortened and long bones angulated. At 19 hours of age, clinical seizures developed and were confirmed by amplitude integrated electroencephalography (aEEG). The aEEG background was discontinuous, a pattern indicating encephalopathy. Seizures were unresponsive or partially responsive to treatment with phenobarbital, phenytoin and midazolam. Both seizures and encephalopathy resolved when pyridoxine and phenobarbital were used in combination. Diagnosis was made after urine metabolic screening by tandem mass spectrometry (MSMS) showed elevated levels of phosphoethanolamine (PEA). This was confirmed by measuring PEA levels in urine using ion exchange chromatography $(828 \mu \mathrm{mol} / \mathrm{mmol}$ of creatinine, reference range: $<25)$. Serum ALP activity was low $(<20 \mathrm{IU} / \mathrm{L}$, reference range: 100 to $350 \mathrm{IU} / \mathrm{L}$ ) consistent with the diagnosis of hypophosphatasia. Secondary pyridoxine deficiency was indicated by increased levels of urine vanillactic which normalized with pyridoxine treatment. The patient died following complications of the associated seizure disorder. Patient B has a milder form and presented initially with skeletal dysplasia and sepsis. The urine metabolic screen showed an elevated level of PEA ( $199 \mu \mathrm{mol} / \mathrm{mmol}$ creatinine). This patient had a slightly higher ALP (30 IU/L), normal vanillactic levels and is currently well. These two patients illustrate the broad phenotypic spectrum of hypophosphatasia and the fact that some patients may exhibit subtle clinical signs. The fact that the initial diagnosis in the patients was made from a urine metabolic screen, highlights the importance of including PEA testing in urine screening, especially for infants with skeletal dysplasia and/or seizures.

\section{'FOOD IS MY MEDICINE': THE CHALLENGES OF TURNING DIETARY THERAPY INTO FOOD INFORMATION IN INBORN ERRORS OF METABOLISM}

\section{L.Vandervliet}

Department of Nutrition and Dietetics, Sydney Children's Hospital, Sydney, Australia

Inborn errors of metabolism are a group of rare diseases where dietary therapy is often the primary therapy. Dietary education includes daily management as well as managing illness, nutritional adequacy, and practicalities of the diet such as ordering special formulas and foods. Compliance to the diet is essential as consequences can be life threatening if not followed. The aim is to describe the challenges in dietary education and management of a child with a urea cycle disorder. The reference case is a now 20-month-old male with citrullinemia diagnosed at birth, with an initial ammonia of $2975 \mathrm{~mol} / \mathrm{L}$. Since diagnosis the child has had four admissions to hospital with hyperammonemia. Education and compliance with dietary therapy has been challenging due to psychosocial factors including language and parental education level. A variety of teaching approaches have been used to manage this child and empowerment of the family has been difficult. Techniques have included: visual feeding plans, formula room demonstrations, food models, supermarket tours, written quizzes, a folder for all written information, frequent phone calls and bedside signs for inpatient admissions to prevent feeding of inappropriate foods. Education to nursing staff has also been necessary to manage inpatient admissions. Total dietetic time for direct interventions with this child has been 62 hours. Inborn errors of metabolism present a challenging case for dietitians. Dietary management requires the dietitian to take on an educational role, usually with parents. This case shows this can be a lengthy, time consuming process. Practical experience in addition to visual aids is likely to be more successful than visual aids alone. ${ }^{1}$ Barriers to compliance can include parental education level and maternal knowledge ${ }^{2}$ as well as attitudes and behaviors. ${ }^{3,4}$ To achieve long term compliance the dietitian needs to use kills in motivational interviewing and counseling as well as dietary education.

${ }^{1}$ Journal of Human Nutrition and Dietetics, 2009; 22:409-413.

${ }^{2}$ Journal of Human Nutrition and Dietetics, 2008; 21:351-358.

${ }^{3}$ Journal of Human Nutrition and Dietetics, 2008; 21:474-485.

${ }^{4}$ Journal of the American Dietary Association, 2010; 110:247-252

\section{CHROMOSOME CNVS DETECTED BY ARRAY CGH: SEXY, VEXING CHANGES}

W.Waters', J. Nicholl', E. Haan ${ }^{2}$, J. Liebelt ${ }^{2}$, C. Barnett ${ }^{2}$, D. Bratkovic ${ }^{3}$, S.Yu' ${ }^{1}$ Genetics and Molecular Pathology, Cytogenetics, SA Pathology, WCH, Adelaide, Australia

${ }^{2}$ Genetics and Molecular Pathology, SA Clinical Genetics Service, SA Pathology, WCH, Adelaide, Australia

${ }^{3}$ Genetics and Molecular Pathology, Unit Head, Metabolic Clinic, WCH, Adelaide, Australia

Array CGH studies on 1200 patients screened for intellectual disability, developmental delay, autism, and/or congenital abnormalities have revealed a number of $\mathrm{X}$ chromosome copy number variants (CNVs). Approximately $5.4 \%$ of all changes investigated were on the $\mathrm{X}$ chromosome and fall into three broad categories - incidental, pathogenic and of unclear clinical significance. The interpretation of some of these CNVs on the $\mathrm{X}$ chromosome have proven challenging. Approximately $1.3 \%$ of all X chromosome CNVs investigated were considered to be incidental findings. Gene deletions in the STS (steroid sulfatase deficiency) gene and EDA (ectodermal dysplasia) gene included. Pathogenic X chromosome CNVs account for approximately $2.3 \%$. Clinically relevant gene deletions in ATP7A (Menke's disease), CDKL5 (early infantile epileptic encephalopathy 
2), NHS (Nance-Horan syndrome) and duplications in MECP2 (Rett syndrome) were identified, with some CNVs as small as $8 \mathrm{~kb}$. Intronic ZNF41 deletions, the contribution of $\mathrm{X}$ chromosome CNVs to a $\mathrm{fra}(\mathrm{X})$ phenotype and an apparent position effect on the PLP1 gene (Pelizaeus-Merzbacher disease) have proven to be challenging in interpretation. A further $1.9 \%$ of all investigated X chromosome $\mathrm{CNV}$ s remain of unclear clinical significance.

\section{CHILDREN WITH NON PHENYLKETONURIA- HYPERPHENYLALANEMIA: MANAGEMENT AND PROBLEMS}

P.Watson', B. Dennison', Z. Junek ${ }^{2,4}$, S. Garnett ${ }^{3}$, V.Wiley ${ }^{2}$, S. Thompson ${ }^{1,4}$, J. Christodoulou 4,5

${ }^{1}$ Nutrition and Dietetics, The Children's Hospital at Westmead, Sydney, Australia

${ }^{2}$ New South Wales Newborn Screening Programme, The Children's Hospital at Westmead, Sydney, Australia

${ }^{3}$ Kid's Research Institute, The Children's Hospital at Westmead, Sydney, Australia ${ }^{4}$ Western Sydney Genetics Program, The Children's Hospital at Westmead, Sydney, Australia

${ }^{5}$ Disciplines of Paediatrics and Child Health and Genetic Medicine, The University of Sydney, Australia

The New South Wales Newborn Screening Programme identified 147 children with elevated blood phenylalanine (phe) between January 1998 and December 2010. Of these, 42 (29\%) were diagnosed as hyperphe (62\% male) based on two subsequent blood spot tests (180 to $400 \mu \mathrm{mol} / \mathrm{L}$ ) and normal urinary pterins and neurotransmitters. For this group median blood phe levels for the first, second and third blood tests were 210,250 and $195 \mu \mathrm{mol} / \mathrm{L}$ respectively. The current management protocol recommends treatment starts following 2 or 3 levels > $350 \mu \mathrm{mol} / \mathrm{L}$ when well. Since 1998,18 children ( $43 \%$ cohort) recorded results $>350 \mu \mathrm{mol} / \mathrm{L}$ and $\leq 600 \mu \mathrm{mol} / \mathrm{L}$ while 5 children (12\% cohort) recorded at least one blood level $>600 \mu \mathrm{mol} / \mathrm{L}$, the highest being $1450 \mu \mathrm{mol} / \mathrm{L}$. Six children were prescribed a proteinrestricted diet with a phe-free supplement (AA sup), aged 5-48 weeks, with blood phe range of $450-1290 \mu \mathrm{mol} / \mathrm{L}$. Despite continuous follow-up and education, 2 were not consistently consuming AA sup. Parents of 2 children were counting natural protein $(0.5 \mathrm{~g} / \mathrm{kg} /$ day $)$ while parents of 4 could not quantify the degree of protein restriction. Perceived barriers to effective dietary treatment included refusal of the late introduced AA sup and an inability of parents to manage dietary protein restriction. Our concerns include the impact of persistently elevated blood phe levels on childhood development, nutritional adequacy through dietary protein restriction in the absence of AA sup, the tendency for these patients to 'slip under the clinical radar', and the difficulties of changing established food preferences in young children. It would be beneficial to identify those at risk of elevated levels early. Using Receiver Operating Characteristics Analysis (ROC) the optimal cut point based on the mean of first level of this cohort is 230 $\mu \mathrm{mol} / \mathrm{L}$. This point would have flagged 9 of 14 patients as being 'at risk' of levels $>350 \mu \mathrm{mol} / \mathrm{L}$ in the future.

NUTRITIONAL MANAGEMENT OF A WOMAN WITH MAPLE SYRUP URINE DISEASE DURING PREGNANCY, LABOUR AND POSTPARTUM.

\author{
M.Westbrook', M.Tchan', B.Wilcken ${ }^{2}$ \\ ${ }^{1}$ Genetic Medicine, Westmead Hospital, Sydney, Australia \\ ${ }^{2}$ Biochemical Genetics Service, Children's Hospital at Westmead, Sydney, Australia
}

Maple syrup urine disease (MSUD) is an autosomal recessive disorder caused by a deficiency of branched chain keto-acid dehydrogenase. We describe the dietary management and successful outcome of pregnancy of a woman with MSUD. The patient was diagnosed with MSUD at 10 days of age. She had several significant decompensations during her first few years of life and was left with mild intellectual impairment. She presented to the adult genetic metabolic clinic at Westmead Hospital, with her husband at 10 weeks gestation. The patient was educated initially on a diet providing $60 \mathrm{~g}$ protein from a branch chain amino acid (BCAA) free supplement and $23 \mathrm{~g}$ natural protein/day. Over the course of the pregnancy the amount of natural protein was adjusted based on the results of regular monitoring of BCAA levels via dried blood spot analysis. The aim was to achieve a plasma leucine level $<300 \mu \mathrm{mol} / \mathrm{L}$ and preferably within the normal range. Consultation with the patient and her husband was via face to face, mobile telephone and email with verbal instructions confirmed in writing. The patient had problems counting protein and keeping a food diary so dietary instruction was based on changes in food intake rather than grams of protein. The birth management plan was drawn up by the genetic metabolic specialist in consultation with the obstetrician, midwife and dietitian to ensure the patient received sufficient kilojoules and a diet low in natural protein to avoid decompensation during labour and postpartum. The patient gave birth to a healthy baby and was discharged on day 8 postpartum. The patient initially breastfed her baby, but when she developed mastitis on day 12 postpartum and her plasma leucine level rose to $549 \mu \mathrm{mol} / \mathrm{L}$ she decided to wean her baby to avoid a recurrence of mastitis and the risk of developing decompensation.

\section{NEWBORN HEALTHY HEARING PROGRAM: A REVIEW OF GENETIC COUNSELING SERVICES PROVIDED}

\section{J.A.White, A. Hattam}

Genetic Health Queensland RBWH, Queensland Health, Brisbane, Australia

Queensland Health via the Healthy Hearing Program has been offering newborn hearing screening to all children born in public and private hospitals throughout Queensland since 2006. The aim of this program is to detect permanent hearing loss (PHL) in babies before they reach 3 months of age to ensure appropriate diagnosis and early intervention. It was recognized in 2007 that a number of existing services, including Clinical Genetics, could provide input into this program. To promote collaboration and service development, the Healthy Hearing Program provided Genetic Health Queensland (GHQ) with recurrent funding for provision of clinical genetics services. We will present an audit of GHQ service delivery to the Healthy Hearing Program over a period of 3 years from July 2007 to June 2010. The aim was to focus on areas such as numbers of referrals/consultations; types of hearing loss; what testing was initiated; and whether a diagnosis was made. Utilization of GHQ Services by the Healthy Hearing Program will be discussed in detail and possible improvements to service delivery will be outlined.

\section{A NOVEL UBE3A VARIANT IN TWO SIBLINGS WITH ANGELMAN SYNDROME}

M.Williams', H. Heussler' ${ }^{2}$, M. Kovalcin ${ }^{3}$, D. Zand ${ }^{3}$, D. Cowley', J. Harraway',

I. McGown'

${ }^{1}$ Molecular Genetics, Mater Pathology, Brisbane, Australia

${ }^{2}$ Mater Children's Hospital, Brisbane, Australia

${ }^{3}$ Children's National Medical Centre, Washington, DC, United States

Angelman syndrome (AS, OMIM\# 105830) is characterized by severe developmental delay, absent or severely limited speech, gait ataxia and/or tremulousness of the limbs, and a unique behavioral phenotype, with a happy demeanour that includes frequent and sometimes inappropriate laughter, smiling, and excitability. Disease mechanisms for AS include de novo maternally derived deletions of 15q11-q13 (70-75\% of cases), paternal uniparental disomy (UPD; 3$7 \%)$, or imprinting defects affecting the maternal chromosome (2-3\%). Additionally, $\sim 10 \%$ of cases are caused by point mutations in the maternally inherited/expressed E6-AP ubiquitin-protein ligase gene (UBE3A). We present two siblings, one 4-year-old male and one 3-year-old female. Both patients had signs and symptoms suggestive of AS, including hypotonia, developmental delay and ataxia. Methylation-Sensitive Multiplex Ligation-Dependent Probe Amplification (MS-MLPA) of the 15q11-q13 region showed a normal methylation pattern and normal copy number in both siblings. SNP array analysis at a resolution of $50 \mathrm{~kb}$ showed no copy number abnormalities or loss of heterozygosity. UBE3A gene sequencing was performed, and a novel variant was detected in both siblings (UBE3A:c.1505T>A p.Leu502His). This amino acid is 
highly conserved across many species. Parental studies confirmed that this mutation was maternally inherited, suggesting a diagnosis of AS in these siblings. Further family studies showed that no other family members carried this variant or displayed any AS characteristics. Functional studies are not available at this time. In conclusion, we have described a novel potentially pathogenic variant in the UBE3A gene which was maternally inherited and co-segregated in two siblings exhibiting an Angelman syndrome phenotype.

\section{AN INCIDENTAL FINDING OF A DELETION OF TWO EXONS IN THE DMD GENE IN A FEMALE AS DETECTED BY MOLECULAR KARYOTYPING WITH AFFYMETRIX 2.7M SNP MICROARRAY}

M.Williams, J. Harraway, M. Doody

Molecular Genetics, Mater Pathology, Brisbane, Australia

Becker muscular dystrophy (BMD; OMIM\# 300376) presents as a mild form of Duchenne muscular dystrophy with proximal muscle weakness and wasting. It is an X-linked disease with only $5-10 \%$ of females exhibiting the characteristic muscle weakness. Our patient, a 6-month-old female, presented with developmental delay and hypotonia. Molecular testing using the Affymetrix $2.7 \mathrm{M}$ chip detected a deletion of exons 43 and 44 in the dystrophin gene (DMD) located at $\mathrm{Xp} 21.2$ as an incidental finding. As genome wide microarray analysis becomes more common in the evaluation of developmental delay and intellectual impairment, the incidence of detection of unexpected but relatively common diseases will increase. The implications of this result for the patient and the patient's family are discussed.

\section{TWO NOVEL GLUCOKINASE GENE (GCK) MUTATIONS CAUSING MATURITY ONSET DIABETES OF THE YOUNG (MODY) IN AUSTRALASIAN PATIENTS}

\author{
M.Williams', M. Harris'2, A. Cotterill2, D. Cowley', I. McGown' \\ ${ }^{1}$ Molecular Genetics, Mater Pathology, Brisbane, Australia \\ ${ }^{2}$ Mater Children's Hospital, Brisbane, Australia
}

Maturity onset diabetes of the Young (MODY; OMIM \#606391) is a group of disorders that affects about $2 \%$ of people with diabetes mellitus. MODY is often not recognized and, as a result, affected individuals may be treated as Type 1 or Type 2 diabetics by their doctors. MODY has four main characteristics:

- diabetes presents at a younger age, usually less than 25 years old

- non-ketotic presentation

- beta-cell dysfunction (People with MODY do not produce enough insulin; this is different from Type 2 diabetes where people are insulin resistant)

- autosomal dominant inheritance.

The GCK gene encodes an intracellular enzyme that senses glucose and coverts it into Glucose-6-phosphate, which then undergoes glycolysis in the mitochondria to produce ATP. Intracellular potassium is pumped out of the cell using energy from ATP, which in turn leads to calcium influx into the cell and the release of insulin. Heterozygous inactivating mutations in the GCK gene cause MODY, subtype glucokinase (GCK-MODY; MODY2; OMIM\# 125851). GCK-MODY is caused by impaired glucose sensing by the beta-cell, resulting in a mild, nonprogressive hyperglycemia (as measured by oral-glucose tolerance test) that is often asymptomatic. The blood glucose threshold for secretion of insulin is raised in the presence of these mutations. Only a small percentage of patients require insulin therapy and diabetes-associated complications are rare. Diagnosis of patients with GCK-MODY is important, as about $50 \%$ of female carriers will develop gestational diabetes and infants born to non-affected mothers may have a reduced birthweight. Here we describe two novel GCK gene mutations.
'THERE'S NO EASY ANSWER': AGE AT DIAGNOSIS OF BOYS WITH DUCHENNE MUSCULAR DYSTROPHY AND PARENT'S VIEWS ON POPULATION SCREENING

S. H.Wong ${ }^{1,2}$, A. D.Archibald', B. J. McClaren', A. J. Kornberg ${ }^{1,3,4}$, S.A. Metcalfe ${ }^{1,3}$

1 Murdoch Childrens Research Institute, Royal Children's Hospital, Melbourne, Australia

2 Department of Medicine, The University of Melbourne, Australia

3 Department of Paediatrics, The University of Melbourne, Australia

4 Department of Neurology, Royal Children's Hospital, Melbourne, Australia

Newborn screening (NBS) for Duchenne muscular dystrophy (DMD) is potentially controversial as there is no treatment that can be implemented at birth, and the pros and cons are subject to debate. We conducted a mixed methods study to: (1) identify the age at which boys are diagnosed with DMD in Victoria from 2005-2010; and, (2) to explore views of parents of boys with DMD with regard to population screening for DMD in Victoria. To achieve these aims an audit of laboratory records for all tests ordered for DMD from 2005-2010 $(n=$ 77) was undertaken and semi-structured interviews with 15 parents of individuals with DMD were conducted. The audit revealed that the mean age at diagnosis and at testing for DMD was 5 years; this age has not changed substantially over the period investigated. The age at diagnosis reported by parents who were interviewed ranged from 4 weeks to 8 years and the interviews demonstrated a $1-2$ year gap between initial concerns about the child's development and receiving the DMD diagnosis; all found this delay in diagnosis to be very difficult. Most participants perceived screening for DMD to be acceptable as this would allow: implementation of early interventions; and guidance with financial, practical and reproductive decision-making. Participants generally preferred screening in infancy (6-36 months) and opposed NBS as they felt that a diagnosis in infancy would have less impact on the development of the parent-child relationship and that parents may be beginning to notice signs of DMD at this time. Other considerations raised by parents included: the impact of an earlier diagnosis on parenting the child; having a period of 'normality'; and resource implications of screening. Based on these data we are currently developing a model of offering population screening for genetic causes of developmental delay in infants.

\section{CAMURATI-ENGELMANN DISEASE PRESENTING AS ANOREXIA IN A YOUNG CHINESE GIRL}

K. H.C.Wu', A. Turner ${ }^{2}$, M. Kohn ${ }^{3}$, D. Sillence ${ }^{4}$

${ }^{1}$ Genetic Medicine, Westmead Hospital, Sydney, Australia

${ }^{2}$ Clinical Genetics, Sydney Children's Hospital Network (Randwick), Sydney, Australia

${ }^{3}$ Eating Disorders Service, Sydney Children's Hospital Network (Westmead), Sydney, Australia

${ }^{4}$ Connective Tissue Dysplasia Management Service, Sydney Children's Hospital Network (Westmead), Sydney, Australia

Purpose: Camurati-Engelmann disease (CED) is a rare autosomal dominant condition characterized by progressive and painful muscle weakness and hyperostosis of long bones and skull; and caused by mutations in the transforming growth factor-beta 1 (TGFB1) gene. We report a 10-year-old Chinese girl whose long-standing symptoms typical of CED had been previously attributed to psychological stress and an eating disorder. Methods: Case report on clinical presentation, radiographic findings, molecular genetic testing, treatment, and counseling issues. Results: The proband presented with a 5-year history of anorexia poorly responsive to psychotropic medication and intermittent hospitalization for enteral feeding. During her most recent admission, she was noted to have a waddling gait with a long-standing history of abnormal gait, proximal muscle weakness, severe lower limb pain, recurrent headaches, and urinary and fecal incontinence. There was no relevant family history. On examination, she had a BMI 11, proximal muscle weakness, significantly reduced muscle mass and subcutaneous fat. The skeletal survey showed extensive sclerosis in the diaphyses of the long bones (including both femora, tibiae, fibulae, humeri and radii) and the skull base, with sparing of hands/feet and the vertebrae, characteristic of CED. Genetic testing of TGFB1 is underway. She was 
commenced on Deflazacort $(0.9 \mathrm{mg} / \mathrm{kg} /$ day for 3 months and weaning), which is effective in relieving symptoms such as pain and fatigue in patients with CED $1-5 ; 1^{1-5}$ and penicillamine $(2.5$ $\mathrm{mg} / \mathrm{kg} / \mathrm{day}$ ), which based on our previous experience has resulted in clinical and radiological improvement. She responded dramatically in terms of reduced pain, muscle weakness and incontinence to these medications with minimal side effects (normal full blood count, electrolytes and liver function monitoring). Counseling challenges are discussed. Conclusion: Our case demonstrates a relatively common presentation of a rare problem; and good symptomatic response to steroid therapy as previously published, and to penicillamine, with minimal side effects.

${ }^{1}$ Journal of Paediatric and Child Health 1999; 35:401-405.

${ }^{2}$ Pediatrics 1985; 75:321-323.

${ }^{3}$ Journal of Rheumatology 1985; 12:809-813.

${ }^{4}$ Clinical Rheumatology 1995; 14:485-486.

${ }^{5}$ Clinical Nuclear Medicine 2001; 26:680-682.

DETERMINING THE CAG REPEAT SIZE OF A LARGE MATERNALLY TRANSMITTED EXPANSION IN HUNTINGTON DISEASE

D. B.Young, V. Hyland

Molecular Genetics, Pathology Queensland, Brisbane, Australia

In Huntington's disease anticipation upon maternal transmission is rare. Here we report the case of a 3-year-old girl with a maternal family history of Huntington's disease. The child presented with ataxia, tremor, dysarthria and sialorrhea. Juvenile-onset Huntington disease is usually transmitted paternally and is associated with very large expansions of the CAG repeat. The child's mother developed symptoms of Huntington's disease at the age of 22. When the mother was originally diagnosed, molecular analysis determined that she had an expansion of 65 CAG repeats in the Juvenile-onset range. Targeted mutation analysis of the child's DNA by PCR initially identified a single normal CAG repeat of 17 . Subsequent TP-PCR analysis indicated the presence of a very large repeat expansion. With modification of PCR reaction conditions it was possible to size the child's expansion to be 185 CAG repeats (+/- 10 repeats). These results demonstrate that very large expansions can occur through the maternal lineage if the mother carries an expansion of sufficient size, and that these expansions can be detected and sized by PCR analysis.
THE EXPERIENCES OF RESEARCH PARTICIPANTS OFFERED GENETIC TESTING INFORMATION AS A RESULT OF TAKING PART IN A POPULATION-BASED OVARIAN CANCER RESEARCH STUDY

M.Young', S.Wake ${ }^{2,3}$, K.Alsop', D. Bowtell', G. Mitchell', T.AOCS Study Group ${ }^{4,5}$, L. Plunkett ${ }^{2}, \mathrm{~A}$. Crook ${ }^{2}$

1 Peter MacCallum Cancer Centre, Melbourne, Australia

2 Department of Paediatrics, University of Melbourne, Australia

3 Murdoch Childrens Research Institute, Genetic Health Services Victoria, Melbourne, Australia

4 Westmead Institute for Cancer Research, University of Sydney at Westmead, Australia 5 Queensland Institute for Medical Research, Brisbane, Australia

Background: The Australian Ovarian Cancer Study (AOCS) is a population based study. Between 2002 and 2006, women with invasive ovarian cancer were recruited with 1,273 providing a blood sample. BRCA1 or BRCA2 mutation testing has been undertaken in this cohort. Women in whom a mutation has been identified, or their next of kin in the case where the woman is deceased, have been notified in writing (notification letter) by the researchers about the finding of a mutation and the availability of obtaining these results through a family cancer clinic. The AOCS Psychosocial project has interviewed individuals who received notification letters. Aims of AOCS psychosocial study: (1) Explore individuals' understanding of information contained in the letter they received from the researchers; (2) Determine what informs individuals' decisions about whether or not to contact a familial cancer clinic and take up genetic testing information. Results: A total of 15 in-depth interviews have been undertaken to date. Participants' responses to the notification letter and their understanding of the letter varied. Genetic responsibility was a key facilitator to individuals obtaining the results through a family cancer clinic. Barriers included wanting to protect others, experiential knowledge and ambivalence. The implications for future research studies and clinical genetic counseling practice will be explored. 